\title{
Indacenodipyrene Containing Small Molecules and Ladder Polymers
}

\author{
Ain Uddin and Kyle N. Plunkett \\ ${ }^{*}$ School of Chemical and Biomolecular Sciences and the Materials Technology Center, Southern Illinois \\ University, Carbondale, IL 62901 United States
}

Dedicated to the memory of Susan A. Odom.

Abstract:

A series of s-indaceno[1,2,3-cd:5,6,7-c'd']dipyrene-containing small molecule and ladder polymers were prepared using a palladium catalyzed arylation reaction. Precursor polymers and their resulting ladder polymers with molecular weights up to $13 \mathrm{kDa}$ were prepared. The rigid, planar materials possessed highest occupied molecular orbital (HOMO) energies of -5.39 to -5.23 $\mathrm{eV}$, lowest unoccupied molecular orbitals (LUMO) energies of $-2.42 \mathrm{eV}$ to $-2.98 \mathrm{eV}$, and optical gaps of 1.68 to $2.03 \mathrm{eV}$. Organic field effect transistors were prepared with derivatives giving hole mobilities up to $2.5 \times 10^{-5} \mathrm{~cm}^{2} \mathrm{~V}^{-1} \mathrm{~s}^{-1}$.

\section{Introduction}

The demand of renewable and sustainable energy has grown exponentially due to industrialization, urbanization, geopolitical changes, and environmental concerns. ${ }^{1-3}$ Robust organic semiconducting materials that are thermally and oxidatively stable while possessing high charge carrier mobilities are worthy targets for advancing technologies including organic field effect transistors and solar cells. ${ }^{4,5}$ Among the variety of organic materials possible for adaptation to these technologies, conjugated ladder polymer based semiconductors offer opportunities for improved thermal stability and greater interchain pi-stacking interactions owing to the lack of bond rotation along the polymer backbone. ${ }^{6-22} \quad \mathrm{New}$ synthetic pathways that enable the synthesis of unique carbon backbones in conjugated ladder polymers can provide opportunities to probe the structure-function properties and enable advancement in this field.

In this contribution, we have synthesized a series of rigid conjugated small molecules and conjugated ladder polymers based on an indacenodipyrene skeleton (Figure 1). The pyrene chromophores provide a large surface area aromatic chromophore that is well-studied in its monomeric form. In addition, this work builds upon a different pyrene-fused s-
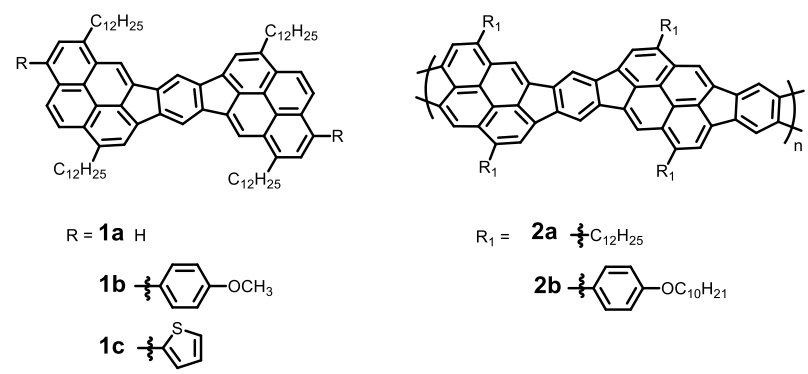

Figure 1: Conjugated small molecule and conjugated ladder polymers based on indacenodipyrene.

Scheme 1: Synthesis of small molecule precursor via Suzuki cross coupling.

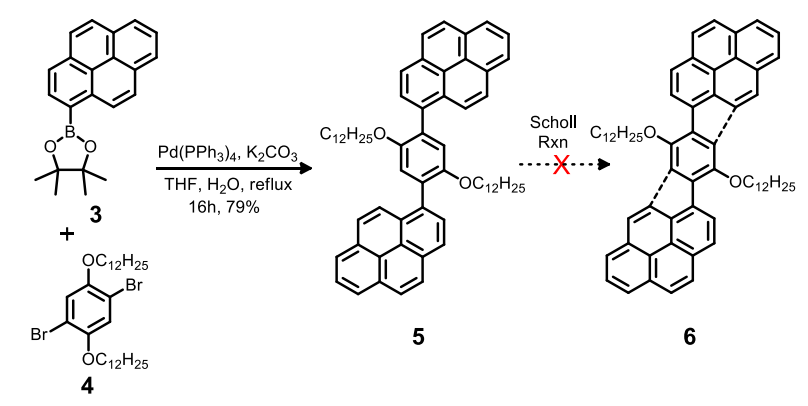
indacene regioisomer recently reported. ${ }^{23} \mathrm{~A}$ 
palladium-catalyzed arylation reaction was employed as an efficient rigidification reaction that ultimately forms five-membered rings as the new ladder rungs linking the pyrene chromophores in the small molecules as well as polymeric materials.

\section{Results and Discussion}

To optimize reaction conditions for the preparation of the conjugated ladder polymers, small molecule derivatives were first prepared. Initial attempts to access the fused indacenodipyrene scaffold followed a Scholl cyclodehydrogenation strategy (Scheme 1). ${ }^{24,25}$ Singly borylated pyrene 3 was cross-coupled with 1,4-dibromo-2,5-bis(dodecyloxy)benzene 4 to give precursor $\mathbf{5}$ in good yields. However, Scholl cyclodehydrogenation reactions utilizing either $\mathrm{FeCl}_{3}{ }^{26}$ or $\mathrm{DDQ}^{27}$ with strong organic acids were found to not give the desired product $\mathbf{6}$, but instead resulted in retrieval of starting material. As an alternative, we prepared brominated precursors to enable a palladium-catalyzed arylation reaction strategy. ${ }^{28,29}$ Mono-brominated pyrene derivatives 7-9 with varying substituents on the pyrene core were prepared (Supporting Information) and reacted with 2,2'-(2,5-dibromo-1,4-phenylene)bis(4,4,5,5-tetramethyl-1,3,2-dioxaborolane) $\mathbf{1 0}^{30}$ to give precursors 11-13 in modest yields of 29-35\%. Here, $\left[\mathrm{Pd}\left(\mathrm{PCy}_{3}\right)_{2} \mathrm{Cl}_{2}\right]$ as the catalyst and DBU as base was employed to create the five-membered ring bridges to give 1a-c in isolated yields of 78$90 \%$. It should be noted that a preliminary route to build up a library of diverse substituted structures from a common precursor was investigated. For example, attempts were made to brominate 1a, which would have provided a scaffold to easily build a library of structures through cross-coupling chemistry (e.g., 1b and 1c). However, these attempts were unsuccessful in preparing pure brominated materials for further utilization. This preliminary difficulty led to the less convenient, yet operational method, of stepwise functionalization we report here.

Scheme 2: Synthesis of small molecule derivatives via Suzuki cross coupling and Heck reaction.

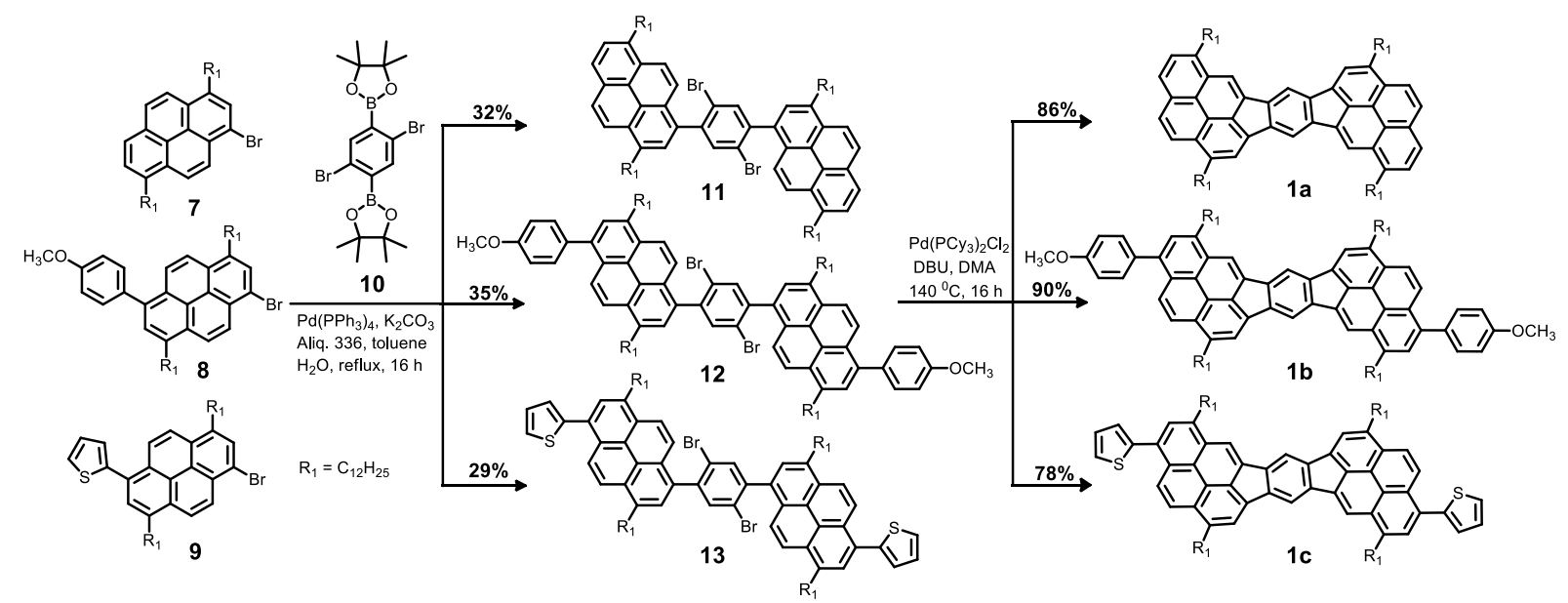

With the small molecule derivatives prepared, the reaction chemistry was applied to preparing ladder conjugated polymers. Pyrene precursors were modified from the single bromine containing monomers 7-9 to dibromo-containing monomers 14 and 15. Polymerization conditions utilized the same Suzuki-Miyaura conditions with monomer $\mathbf{1 0}$ to build up the pyrene containing materials. Recycling preparative gel permeation chromatography (GPC) was used to exclude lower molecular weight material and resulted in collected samples of $10 \mathrm{kDa}$ and $13 \mathrm{kDa}$ (based on GPC with polystyrene standards), for $\mathbf{1 6}$ and 17, respectively. The palladium-catalyzed arylation reaction was applied to $\mathbf{1 6}$ and $\mathbf{1 7}$ to form the laddered polymers $\mathbf{2 a}$ and $\mathbf{2 b}$. As expected, the 
solubility of the resulting materials was significantly reduced owing to the extended planarized surfaces. Ladder polymer $\mathbf{2 a}$ was found to be totally insoluble in common organic solvents such as toluene, chloroform, dichloromethane, tetrahydrofuran and dichloroethane at higher temperatures. However, $\mathbf{2 b}$ was found to give some solubility $(\sim 0.05 \mathrm{mg} / \mathrm{mL})$ in hot chloroform

Scheme 3: Synthesis of conjugated ladder polymers via Suzuki cross coupling and arylation reaction.

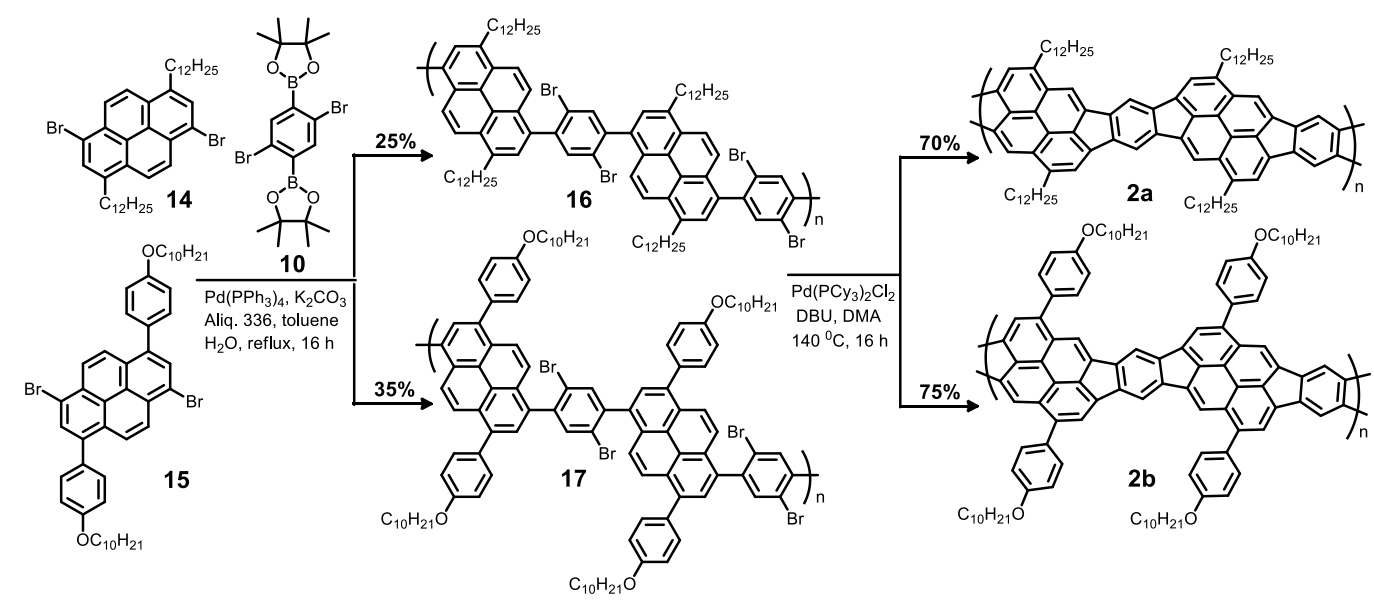

for limited processing and characterization.

Solution based UV/Vis absorption spectra of the indacenodipyrene based materials are shown in Figure 2. Overall, the three small molecule derivatives (1a-c) were similar, but with small bathochromic shifts with substitutions consisting of the anisole $(8 \mathrm{~nm})$ and thiophene $(18 \mathrm{~nm})$. The optical band gaps of the pyrene-based materials, as determined by the onset of filmbased absorption from the diffuse reflectance absorption spectra (supporting information) were $2.03 \mathrm{eV}, 1.98 \mathrm{eV}$ and $1.90 \mathrm{eV}$ for $\mathbf{1 a}, \mathbf{1 b}$ and 1c, respectively. The precursor polymer $\mathbf{1 7}$ onset is significantly hypsochromically shifted in relation to the ring closed compounds 1a-c. However, upon the arylation reaction, polymer 2b shows significant bathochromic shift with an onset of $693 \mathrm{~nm}$ in solution with the lowest energy transition (470-600 $\mathrm{nm}$ ) overlapping with the small molecule analogs. The absorption tails to lower energies and suggests expanded delocalization along the polymer backbone in relation to the dimeric pyrene small molecule derivatives.

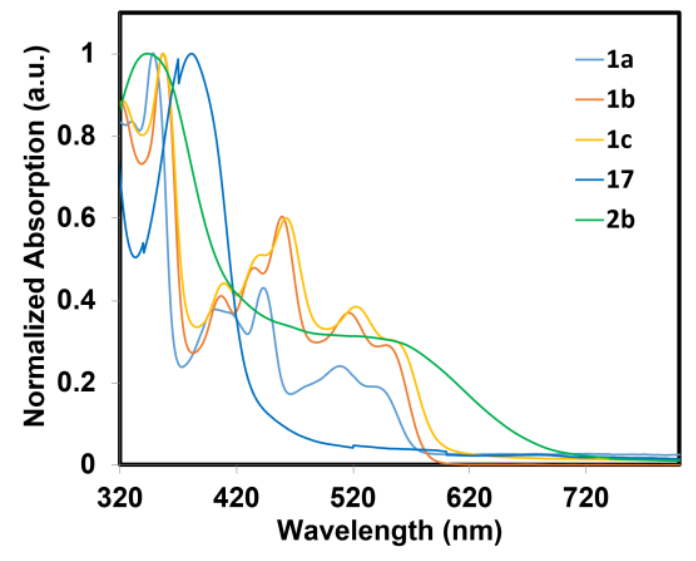

Figure 2: Absorption spectra of small molecules $\mathbf{1 a - c}$ and polymers $\mathbf{1 7}$ and $\mathbf{2 b}$ in chloroform.

To further probe the electronic properties of 1a-c, 16 and 17, cyclic voltammograms $(\mathrm{CV})$ of the solid films were obtained. Organic films for CV were prepared by drop casting chloroform solutions on a glassy carbon electrode. Analysis of the CV shows the materials have both irreversible oxidation and reduction signals (Figure 3). Utilizing ferrocene as an internal standard, the highest occupied molecular level (HOMO) and lowest unoccupied molecular orbital (LUMO) energies of the materials were evaluated. The HOMO levels ranged from $-5.28 \mathrm{eV}$ to $-5.39 \mathrm{eV}$, 
while the LUMO energies varied between -2.42 to $-2.98 \mathrm{eV}$ (Table 1). The optical and electrochemical gaps diverged in absolute value and is presumably owing to the exciton binding energies being considerably different in these systems. ${ }^{31}$

The newly prepared materials were then evaluated in regard to their charge carrier mobilities. Organic field effect transistors with bottom gate and bottom contact arrangements were prepared. Traditional gold contacts deposited on octadecyltrichlorosilane functionalized $\mathrm{Si} / \mathrm{SiO}_{2}$ substrates with an active channel of $30 \mu \mathrm{m}$ were utilized. Films were cast by spin-coating chloroform solutions at 3000 rpm onto the prepared substrates. Typical output and transfer plots of the resulting devices can be found in Figure 4 and Figure 5. The average charge carrier mobilities $\mathbf{1 b}$ and 1c, as tested in air, were found to be an average of $2.5 \times 10^{-5}$ and $7.4 \times 10^{-5} \mathrm{~cm}^{2} \quad \mathrm{~V}^{-1} \mathrm{~s}^{-1}$, respectively. No appreciable current was observed for $\mathbf{1 a}$ or polymer $\mathbf{2 b}$, and were presumed to be owing to inconsistent film formation resulting from poor solubilities. While the charge carrier mobilities in these systems are relatively small, they do demonstrate the materials are active as charge carriers.

In conclusion, we have synthesized a series of new indacenodipyrene based small molecule and conjugated ladder polymers by an efficient palladium catalyzed arylation of a dipyrenyl benzene precursor. The small molecule reaction optimization was applied to make conjugated-ladder polymers that possessed bathochromically shifted optical properties and less soluble materials in comparison to the small molecule analogs. The reaction pathway provides a route to new conjugated ladder materials with modest charge carrier mobilities.

\section{Acknowledgments}

This work was supported by a National Science Foundation CAREER grant (CHE-1352431).

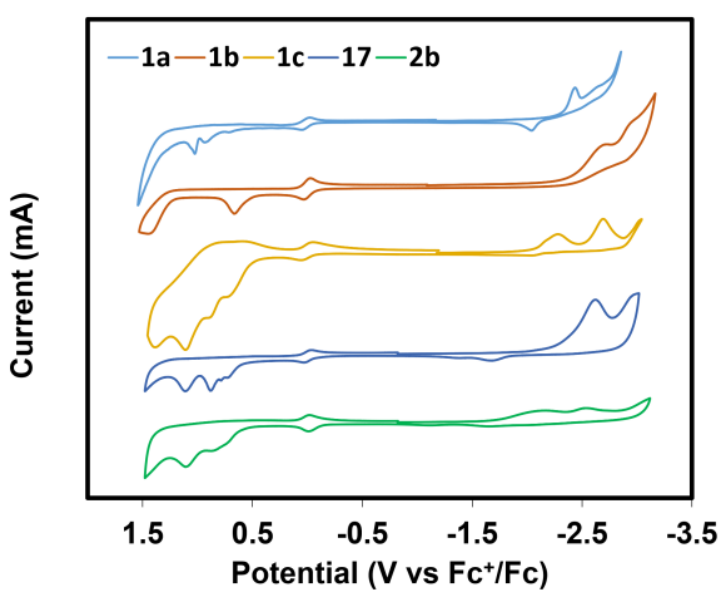

Figure 3: Thin film cyclic voltammetry of 1a-c, 20 and $2 \mathbf{b}$ in acetonitrile with $0.05 \mathrm{M}$ tetrabutyl ammonium hexafluorophosphate, glassy carbon working electrode, platinum counter electrode, and an $\mathrm{Ag} / \mathrm{AgCl}$ reference electrode. Scan rate $=100 \mathrm{mV} / \mathrm{s}$. Ferrocene was added as an internal standard and referenced to $0 \mathrm{~V}$.

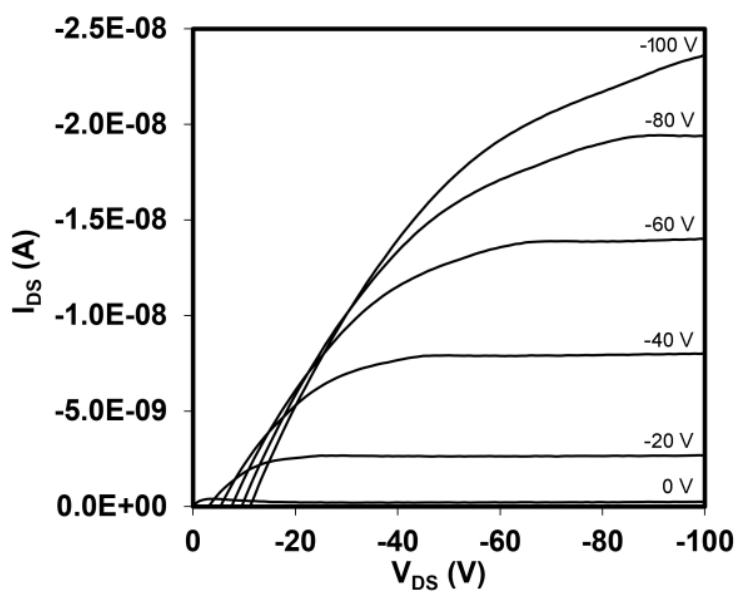

Figure 4: Typical output curves for $\mathbf{1 b}$ showing $\mathrm{p}$-type semiconductor behavior. 


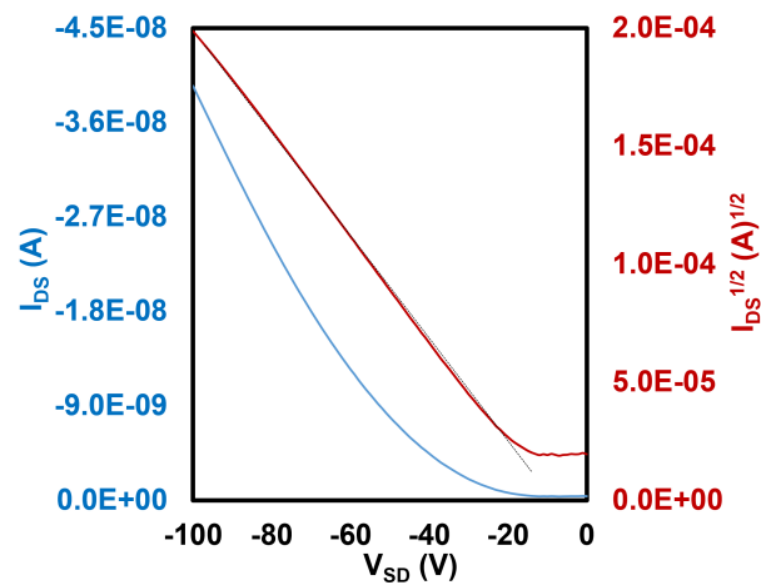

Figure 5: Typical transfer plot for the p-type semiconductor $\mathbf{1 b}$. $\mathrm{V}_{\mathrm{G}}=-80 \mathrm{~V}$. Average mobility $\left(\mathrm{h}^{+}=2.5 \times 10^{-5} \mathrm{~cm}^{2} \mathrm{~V}^{-1} \mathrm{~s}^{-1} \pm 2.1 \times\right.$ $\left.10^{-6}\right)$.

Table 1. Summary of molecular weight and optoelectronic properties of compounds 1a-c, 17 and $\mathbf{2 b}$

\begin{tabular}{cccccccc}
\hline Cmpd & $\begin{array}{c}E_{\text {ox/onset }} \\
(\mathbf{V})\end{array}$ & $\begin{array}{c}E_{\text {red/onset }} \\
(\mathbf{V})\end{array}$ & $\begin{array}{ccccc}\text { HOMO } \\
(\mathbf{e V})\end{array}$ & $\begin{array}{c}\text { LUMO } \\
(\mathbf{e V})\end{array}$ & $\begin{array}{c}\text { E-chem } \\
\text { gap } \\
(\mathbf{e V})\end{array}$ & $\begin{array}{c}\boldsymbol{\lambda}_{\text {onset }} \\
(\mathbf{n m})\end{array}$ & $\begin{array}{c}\text { Optical } \\
\text { gap } \\
(\mathbf{e V})\end{array}$ \\
\hline $\mathbf{1 a}$ & 0.59 & -2.36 & -5.39 & -2.44 & 2.95 & 610 & 2.03 \\
$\mathbf{1 b}$ & 0.43 & -2.38 & -5.23 & -2.42 & 2.81 & 625 & 1.98 \\
$\mathbf{1 c}$ & 0.48 & -2.01 & -5.28 & -2.79 & 2.49 & 651 & 1.90 \\
$\mathbf{1 7}$ & 0.58 & -2.31 & -5.38 & -2.49 & 2.89 & 471 & 2.63 \\
$\mathbf{2 b}$ & 0.58 & -1.82 & -5.38 & -2.98 & 2.40 & 739 & 1.68 \\
\hline
\end{tabular}

${ }^{\text {a }}$ Potentials measured relative to a ferrocenium/ferrocene redox couple used as an internal standard (Figure 3). Ex $_{\text {ox onset }}$ is the onset of oxidation potential, $E_{\text {red/onset }}$ is the onset of reduction potential. HOMO and LUMO values calculated on the basis of the oxidation of the ferrocene reference in vacuum $(-4.8 \mathrm{eV})$. Optical gap taken from $\lambda_{\text {onset }}$ of film (SI). 
References

(1) Griggs, D.; Stafford-Smith, M.; Gaffney, O.; Rockström, J.; Öhman, M. C.; Shyamsundar, P.; Steffen, W.; Glaser, G.; Kanie, N.; Noble, I. Sustainable Development Goals for People and Planet. Nature 2013, 495 (7441), 305-307. https://doi.org/10.1038/495305a.

(2) Lu, L.; Zheng, T.; Wu, Q.; Schneider, A. M.; Zhao, D.; Yu, L. Recent Advances in Bulk Heterojunction Polymer Solar Cells. Chem. Rev. 2015, 115 (23), 12666-12731. https://doi.org/10.1021/acs.chemrev.5b00098.

(3) Polman, A.; Knight, M.; Garnett, E. C.; Ehrler, B.; Sinke, W. C. Photovoltaic Materials: Present Efficiencies and Future Challenges. Science 352 (6283), 307-317.

(4) Anthony, J. E. Functionalized Acenes and Heteroacenes for Organic Electronics. Chem. Rev. 2006, 106 (12), 5028-5048. https://doi.org/10.1021/cr050966z.

(5) Wu, J.; Pisula, W.; Müllen, K. Graphenes as Potential Material for Electronics. Chem. Rev. 2007, 107 (3), 718-747. https://doi.org/10.1021/cr068010r.

(6) Schlüter, A.-D. Ladder Polymers: The New Generation. Advanced Materials 1991, 3 (6), 282-291. https://doi.org/10.1002/adma.19910030603.

(7) Scherf, U.; Müllen, K. Polyarylenes and Poly(Arylenevinylenes), 7. A Soluble Ladder Polymer via Bridging of Functionalized Poly(p-Phenylene)-Precursors. Die Makromolekulare Chemie, Rapid Communications 1991, 12 (8), 489-497. https://doi.org/10.1002/marc.1991.030120806.

(8) Schlüter, A.-D.; Löffler, M.; Enkelmann, V. Synthesis of a Fully Unsaturated All-Carbon Ladder Polymer. Nature 1994, 368 (6474), 831-834. https://doi.org/10.1038/368831a0.

(9) Goldfinger, M. B.; Swager, T. M. Fused Polycyclic Aromatics via Electrophile-Induced Cyclization Reactions: Application to the Synthesis of Graphite Ribbons. J. Am. Chem. Soc. 1994, 116 (17), 7895-7896. https://doi.org/10.1021/ja00096a056.

(10) Scherf, U.; Müllen, K. The Synthesis of Ladder Polymers. In Synthesis and Photosynthesis; Advances in Polymer Science; Springer: Berlin, Heidelberg, 1995; pp 1-40. https://doi.org/10.1007/3-54058908-2_1.

(11) Goldfinger, M. B.; Crawford, K. B.; Swager, T. M. Directed Electrophilic Cyclizations: Efficient Methodology for the Synthesis of Fused Polycyclic Aromatics. J. Am. Chem. Soc. 1997, 119 (20), 4578-4593. https://doi.org/10.1021/ja9642673.

(12) Babel, A.; Jenekhe, S. A. High Electron Mobility in Ladder Polymer Field-Effect Transistors. Journal of the American Chemical Society 2003, 125 (45), 13656-13657. https://doi.org/10.1021/ja0371810.

(13) Xu, C.; Wakamiya, A.; Yamaguchi, S. Ladder Oligo(p-Phenylenevinylene)s with Silicon and Carbon Bridges. J. Am. Chem. Soc. 2005, 127 (6), 1638-1639. https://doi.org/10.1021/ja042964m.

(14) Chen, Z.; Amara, J. P.; Thomas, S. W.; Swager, T. M. Synthesis of a Novel Poly(Iptycene) Ladder Polymer. Macromolecules 2006, 39 (9), 3202-3209. https://doi.org/10.1021/ma052451f.

(15) Briseno, A. L.; Mannsfeld, S. C. B.; Shamberger, P. J.; Ohuchi, F. S.; Bao, Z.; Jenekhe, S. A.; Xia, Y. Self-Assembly, Molecular Packing, and Electron Transport in n-Type Polymer Semiconductor Nanobelts. Chem. Mater. 2008, 20 (14), 4712-4719. https://doi.org/10.1021/cm8010265.

(16) Yang, X.; Dou, X.; Rouhanipour, A.; Zhi, L.; Räder, H. J.; Müllen, K. Two-Dimensional Graphene Nanoribbons. J. Am. Chem. Soc. 2008, 130 (13), 4216-4217. https://doi.org/10.1021/ja710234t.

(17) Plumhof, J. D.; Stöferle, T.; Mai, L.; Scherf, U.; Mahrt, R. F. Room-Temperature Bose-Einstein Condensation of Cavity Exciton-Polaritons in a Polymer. Nature Mater 2014, 13 (3), 247-252. https://doi.org/10.1038/nmat3825.

(18) Lee, J.; Rajeeva, B. B.; Yuan, T.; Guo, Z.-H.; Lin, Y.-H.; Al-Hashimi, M.; Zheng, Y.; Fang, L. Thermodynamic Synthesis of Solution Processable Ladder Polymers. Chem. Sci. 2016, 7 (2), 881889. https://doi.org/10.1039/C5SC02385H.

(19) Yang, W.; Lucotti, A.; Tommasini, M.; Chalifoux, W. A. Bottom-Up Synthesis of Soluble and Narrow Graphene Nanoribbons Using Alkyne Benzannulations. J. Am. Chem. Soc. 2016, 138 (29), 91379144. https://doi.org/10.1021/jacs.6b03014. 
(20) Gao, J.; Uribe-Romo, F. J.; Saathoff, J. D.; Arslan, H.; Crick, C. R.; Hein, S. J.; Itin, B.; Clancy, P.; Dichtel, W. R.; Loo, Y.-L. Ambipolar Transport in Solution-Synthesized Graphene Nanoribbons. ACS Nano 2016, 10 (4), 4847-4856. https://doi.org/10.1021/acsnano.6b00643.

(21) Lee, J.; Kalin, A. J.; Yuan, T.; Al-Hashimi, M.; Fang, L. Fully Conjugated Ladder Polymers. Chem. Sci. 2017, 8 (4), 2503-2521. https://doi.org/10.1039/C7SC00154A.

(22) Cao, Z.; Leng, M.; Cao, Y.; Gu, X.; Fang, L. How Rigid Are Conjugated Non-Ladder and Ladder Polymers? Journal of Polymer Science n/a (n/a). https://doi.org/10.1002/pol.20210550.

(23) Melidonie, J.; Liu, J.; Fu, Y.; Weigand, J. J.; Berger, R.; Feng, X. Pyrene-Fused s-Indacene. J. Org. Chem. 2018, 83 (12), 6633-6639. https://doi.org/10.1021/acs.joc.8b00925.

(24) King, B. T.; Kroulík, J.; Robertson, C. R.; Rempala, P.; Hilton, C. L.; Korinek, J. D.; Gortari, L. M. Controlling the Scholl Reaction. The Journal of Organic Chemistry 2007, 72 (7), 2279-2288. https://doi.org/10.1021/jo061515x.

(25) Grzybowski, M.; Skonieczny, K.; Butenschön, H.; Gryko, D. T. Comparison of Oxidative Aromatic Coupling and the Scholl Reaction. Angewandte Chemie International Edition 2013, 52 (38), 99009930. https://doi.org/10.1002/anie.201210238.

(26) Horibe, T.; Ohmura, S.; Ishihara, K. Structure and Reactivity of Aromatic Radical Cations Generated by FeCl3. J. Am. Chem. Soc. 2019, 141 (5), 1877-1881. https://doi.org/10.1021/jacs.8b12827.

(27) Zhai, L.; Shukla, R.; Wadumethrige, S. H.; Rathore, R. Probing the Arenium-Ion (ProtonTransfer) versus the Cation-Radical (Electron Transfer) Mechanism of Scholl Reaction Using DDQ as Oxidant. J. Org. Chem. 2010, 75 (14), 4748-4760. https://doi.org/10.1021/jo100611k.

(28) Rice, J. E.; Cai, Z. W. An Intramolecular Arene-Triflate Coupling Reaction for the Regiospecific Synthesis of Substituted Benzofluoranthenes. J. Org. Chem. 1993, 58 (6), 1415-1424. https://doi.org/10.1021/jo00058a023.

(29) Reisch, H. A.; Bratcher, M. S.; Scott, L. T. Imposing Curvature on a Polyarene by Intramolecular Palladium-Catalyzed Arylation Reactions: A Simple Synthesis of Dibenzo[a,g]Corannulene. Organic Letters 2000, 2 (10), 1427-1430. https://doi.org/10.1021/ol005755p.

(30) Higashino, T.; Ishida, K.; Sakurai, T.; Seki, S.; Konishi, T.; Kamada, K.; Kamada, K.; Imahori, H. Pluripotent Features of Doubly Thiophene-Fused Benzodiphospholes as Organic Functional Materials. Chemistry - A European Journal 2019, 25 (25), 6425-6438. https://doi.org/10.1002/chem.201900661.

(31) Sariciftci, N. S. Primary Photoexcitations in Conjugated Polymers: Molecular Excitons vs Semiconductor Band Model; World Scientific: Singapore, 1997. 


\title{
Supporting Information
}

\section{Indacenodipyrene Containing Small Molecules and Ladder Polymers}

\author{
Ain Uddin and Kyle N. Plunkett \\ ${ }^{*}$ School of Chemical and Biomolecular Sciences and the Materials Technology Center, Southern Illinois \\ University, Carbondale, IL 62901 United States
}

\section{CONTENTS}

PAGE

1) Diffuse Reflectance Absorption Spectra

SI2

2) Reaction schemes for precursor compounds

SI3-SI5

3) Experimentals

SI6-SI8

4) NMR Spectra

SI19- 


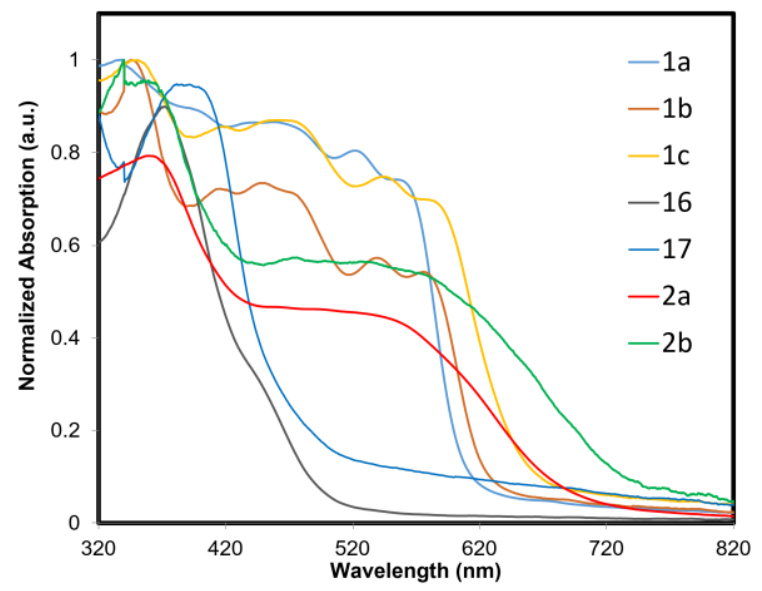

Figure S1. Diffuse reflectance absorption thin film absorption spectra of indacenodipyrene small molecules and polymers. 
Scheme S1: Synthesis of Boronic ester derivatives with benzene and pyrene unit<smiles>CC(C)c1ccc2ccc3cccc4ccc1c2c34</smiles>

3

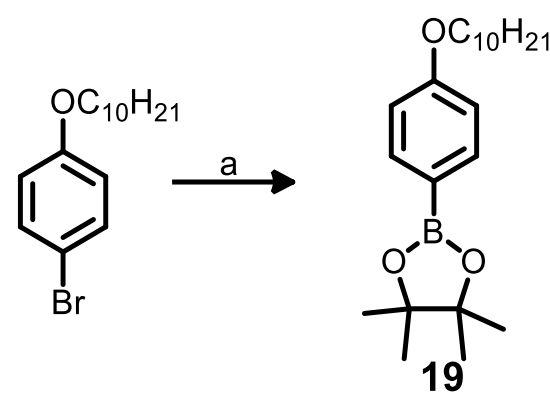<smiles>CC1(C)OB(c2cc(Br)c(B3OC(C)(C)C(C)(C)O3)cc2Br)OC1(C)C</smiles>

10

Conditions: a) bis(pinacolato)diborane, $\mathrm{Pd}\left(\mathrm{PPh}_{3}\right)_{2} \mathrm{Cl}_{2}$, KOAc, 1,4-dioxane, $90{ }^{0} \mathrm{C}, 16 \mathrm{~h}, 79 \%$; b) bis(pinacolato)diboron, 4,4'-di-tert-butyl-2,2'-dipyridyl, (1,5-cyclooctadiene) (methoxy)iridium(I) dimer, cyclohexane, $90{ }^{\circ} \mathrm{C}, 16 \mathrm{~h}, 72 \%$. 
Scheme S2: Synthesis of 1,4-dibromo-2,5-bis(dodecyloxy)benzene<smiles>CCOc1cc(OCC)c(OCC)cc1Br</smiles>

20

21

Conditions: a) 1-bromododecane, $\mathrm{K}_{2} \mathrm{CO}_{3}, \mathrm{CH}_{3} \mathrm{CN}$, argon, reflux, $12 \mathrm{~h}, 85 \%$; b) $\mathrm{Br}_{2}, \mathrm{CH}_{2} \mathrm{Cl}_{2}, 0^{\circ} \mathrm{C}$, rt, overnight, $89 \%$.

Scheme S3: Synthesis of bromide functionalized alkylpyrene derivatives

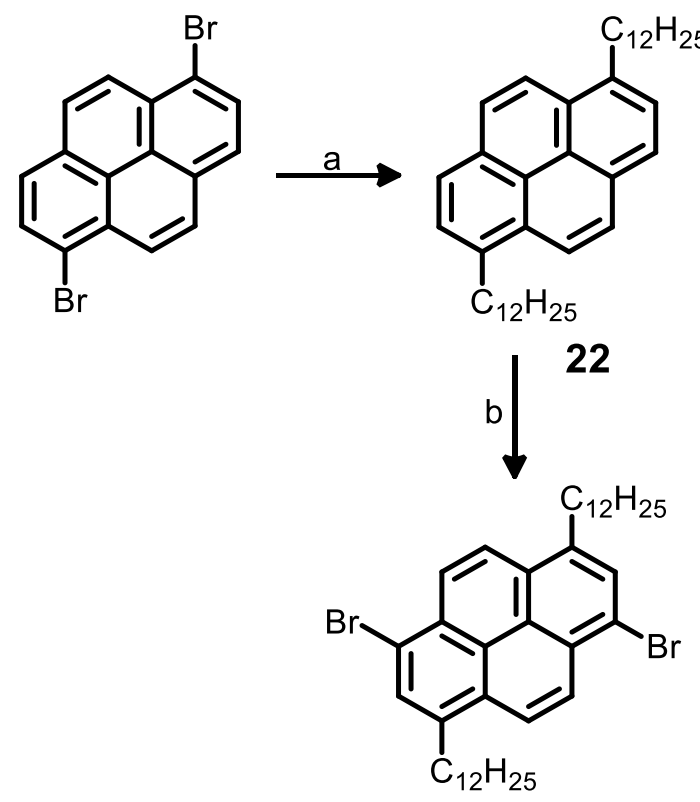

14

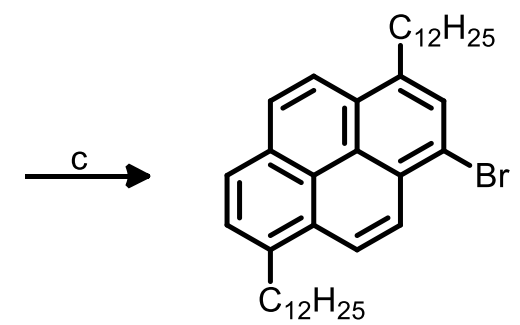

7

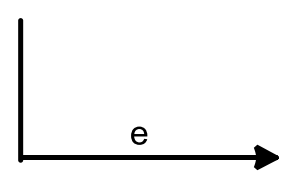

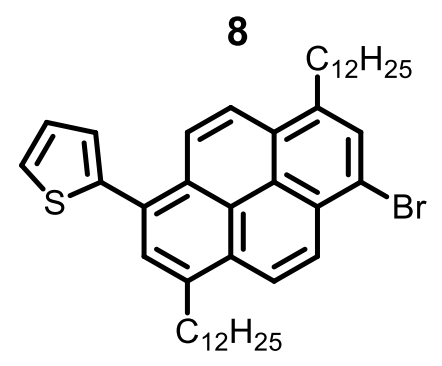

9

Conditions: a) 1-bromododecane, $\mathrm{Mg}$ turnings, 1,6-dibromopyrene, $\mathrm{Ni}(\mathrm{dppp}) \mathrm{Cl}_{2}, 60{ }^{0} \mathrm{C}, 36 \mathrm{~h}$, $72 \%$; b) N-bromosuccinimide, DMF, $\mathrm{CHCl}_{3}$, overnight, $84 \%$; c) N-bromosuccinimide, $\mathrm{NH}_{4} \mathrm{NO}_{3}$, $\mathrm{CHCl}_{3}, 36 \mathrm{~h}, 83 \%$; d) 4-methoxyphenylboronic acid, $\mathrm{Pd}\left(\mathrm{PPh}_{3}\right)_{4}, \mathrm{~K}_{2} \mathrm{CO}_{3}$, THF, $\mathrm{H}_{2} \mathrm{O}$, argon, reflux, 16 h, 67\%; e) 2-(tributylstannyl)thiophene, $\mathrm{Pd}\left(\mathrm{PPh}_{3}\right)_{4}$, toluene, reflux, overnight, $43 \%$. 
Scheme S4: Synthesis of 1,6-dibromo-3,8-bis(4-(decyloxy)phenyl)pyrene

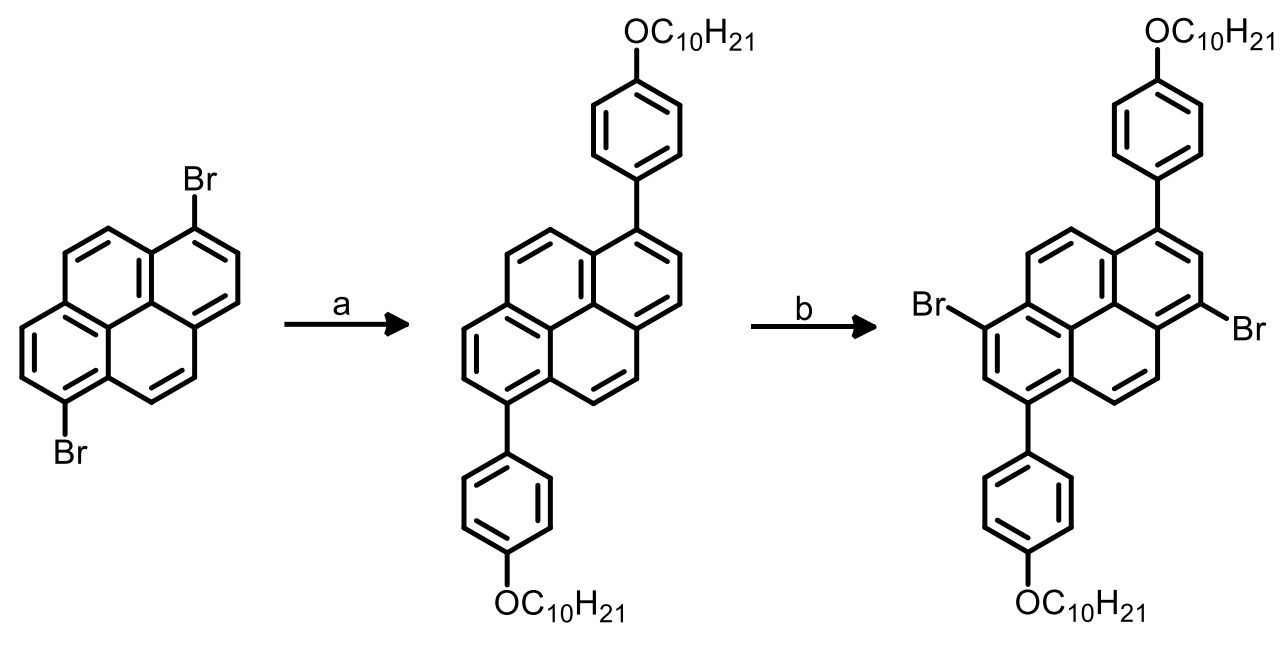

Conditions: a) 3, $\mathrm{Pd}\left(\mathrm{PPh}_{3}\right)_{4}, \mathrm{~K}_{2} \mathrm{CO}_{3}$, THF, $\mathrm{H}_{2} \mathrm{O}$, reflux, argon, overnight; b) N-bromosuccinimide, $\mathrm{DMF}, \mathrm{CHCl}_{3}$, overnight. 


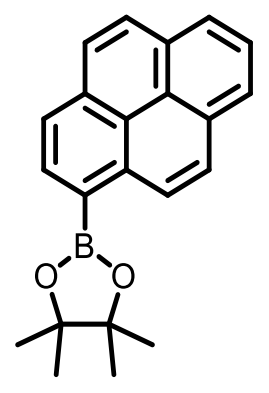

4,4,5,5-tetramethyl-2-(pyren-1-yl)-1,3,2-dioxaborolane (3): In a glove box, 1-bromopyrene (500 mg, $1.80 \mathrm{mmol}), \mathrm{Pd}\left(\mathrm{PPh}_{3}\right)_{2} \mathrm{Cl}_{2}(75 \mathrm{mg}, 0.11 \mathrm{mmol})$, bis(pinacolato)diborane (497 mg, 1.96 $\mathrm{mmol}), \mathrm{KOAc}(611 \mathrm{mg}, 6.22 \mathrm{mmol})$ and $20 \mathrm{~mL}$ dry 1,4-dioxane were added into a seal tube. The reaction mixture was stirred at $90{ }^{\circ} \mathrm{C}$ for $16 \mathrm{~h}$. After the completion of the reaction, the mixture was poured into water, extracted with $\mathrm{CH}_{2} \mathrm{Cl}_{2}(30 \mathrm{~mL} \times 3)$, the resultant organic phase was washed with brine, and dried over anhydrous $\mathrm{Na}_{2} \mathrm{SO}_{4}$. After evaporation of the solvent, the residue was purified by flash column chromatography with hexane and 0 to $40 \%$ dichloromethane to give 420 $\mathrm{mg}(72 \%)$ of 4 as yellowish solid. ${ }^{1} \mathrm{H}$ NMR $\left(400 \mathrm{MHz}, \mathrm{CDCl}_{3}\right) \delta 9.09(\mathrm{~d}, J=9.2 \mathrm{~Hz}, 1 \mathrm{H}), 8.55(\mathrm{~d}$, $J=7.7 \mathrm{~Hz}, 1 \mathrm{H}), 8.14(\mathrm{~m}, J=35.6,26.7,13.4,7.6 \mathrm{~Hz}, 7 \mathrm{H}), 1.51(\mathrm{~s}, 12 \mathrm{H})$.

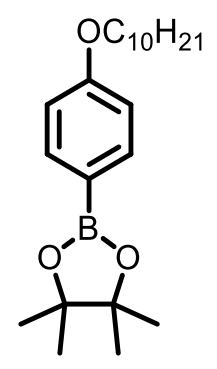

2-(4-(decyloxy)phenyl)-4,4,5,5-tetramethyl-1,3,2-dioxaborolane (19): In a glove box, 1-bromo4-decyloxybenzene $(500 \quad \mathrm{mg}, \quad 1.60 \mathrm{mmol}), \quad \mathrm{Pd}\left(\mathrm{PPh}_{3}\right)_{2} \mathrm{Cl}_{2} \quad(67 \quad \mathrm{mg}, \quad 0.095 \mathrm{mmol})$, bis(pinacolato)diborane (446 mg, $1.76 \mathrm{mmol}), \mathrm{KOAc}(549 \mathrm{mg}, 5.59 \mathrm{mmol})$ and $20 \mathrm{~mL}$ dry 1,4dioxane were added into a seal tube. The reaction mixture was stirred at $90{ }^{\circ} \mathrm{C}$ for $16 \mathrm{~h}$. After the completion of the reaction, the mixture was poured into water, extracted with $\mathrm{CH}_{2} \mathrm{Cl}_{2}(30 \mathrm{~mL} \times 3)$, the resultant organic phase was washed with brine, and dried over anhydrous $\mathrm{Na}_{2} \mathrm{SO}_{4}$. After evaporation of the solvent, the residue was purified by flash column chromatography with hexane and 0 to $40 \%$ dichloromethane to give $456 \mathrm{mg}$ (79\%) of 3 as yellowish liquid. ${ }^{1} \mathrm{H} \mathrm{NMR}$ ( $400 \mathrm{MHz}$, $\left.\mathrm{CDCl}_{3}\right) \delta 7.73(\mathrm{~d}, J=8.6 \mathrm{~Hz}, 2 \mathrm{H}), 6.89(\mathrm{~d}, J=13.1,6.9 \mathrm{~Hz}, 2 \mathrm{H}), 3.99(\mathrm{t}, J=4.5 \mathrm{~Hz}, 2 \mathrm{H}), 1.83-$ $1.74(\mathrm{~m}, 2 \mathrm{H}), 1.47(\mathrm{~d}, J=13.1 \mathrm{~Hz}, 4 \mathrm{H}), 1.33(\mathrm{~s}, 12 \mathrm{H}), 1.28(\mathrm{~m}, J=3.2 \mathrm{~Hz}, 10 \mathrm{H}), 0.89(\mathrm{t}, J=6.9$ $\mathrm{Hz}, 3 \mathrm{H})$. 


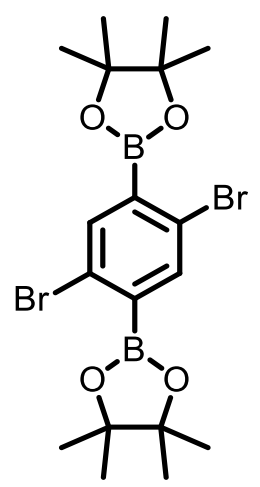

2,2'-(2,5-dibromo-1,4-phenylene)bis(4,4,5,5-tetramethyl-1,3,2-dioxaborolane) (10): In a glove box, 1,4-dibromobenzene (1.00 g, $4.24 \mathrm{mmol})$, (1,5-cyclooctadiene) (methoxy)iridium(I) dimer $(28 \mathrm{mg}, 0.042 \mathrm{mmol}), 4,4^{\prime}$-di-tert-butyl-2,2'-dipyridyl (35 $\mathrm{mg}, 0.13 \mathrm{mmol}$ ), bis(pinacolato)diboron $(2.70 \mathrm{~g}, 10.60 \mathrm{mmol})$ and $25 \mathrm{~mL}$ of cyclohexane were added into a seal tube. The reaction mixture was stirred at $90{ }^{\circ} \mathrm{C}$ for $16 \mathrm{~h}$. After cooling to room temperature, the mixture was filtered. The residue was washed with hexane to afford $1.54 \mathrm{~g}(1.54 \mathrm{~g}, 73 \%)$ of 5 as a white powder. ${ }^{1} \mathrm{H}$ NMR $\left(400 \mathrm{MHz}, \mathrm{CDCl}_{3}\right) \delta 7.74(\mathrm{~s}, 2 \mathrm{H}), 1.37(\mathrm{~s}, 24 \mathrm{H})$.

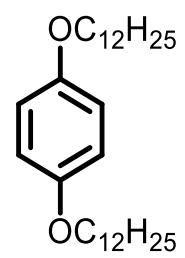

1,4-bis(dodecyloxy)benzene (20): Hydroquinone (1.00 g, $9.08 \mathrm{mmol}$ ), 1-bromododecane (4.97 g, $19.98 \mathrm{mmol})$ and $\mathrm{K}_{2} \mathrm{CO}_{3}(3.77 \mathrm{~g}, 27.3 \mathrm{mmol})$ were dissolved in $20 \mathrm{~mL}$ acetonitrile under an argon atmosphere. The reaction mixture was stirred at reflux for $36 \mathrm{~h}$. the reaction mixture was cooled to room temperature, filtered, and washed with dichloromethane. The filtrate was concentrated, and the residue was purified by silica gel chromatography with a gradient of 0 to $30 \%$ dichloromethane in hexane to give the product as a white solid (85\%). ${ }^{1} \mathrm{H} \mathrm{NMR}\left(400 \mathrm{MHz}, \mathrm{CDCl}_{3}\right)$ $\delta 6.82(\mathrm{~s}, 4 \mathrm{H}), 3.89(\mathrm{t}, J=6.6 \mathrm{~Hz}, 4 \mathrm{H}), 1.79-1.70(\mathrm{~m}, 4 \mathrm{H}), 1.48-1.40(\mathrm{~m}, 4 \mathrm{H}), 1.34-1.22(\mathrm{~m}$, $32 \mathrm{H}), 0.95-0.85(\mathrm{t}, 6 \mathrm{H})$.<smiles>CCOc1cc(Br)c(OCC)cc1Br</smiles>

1,4-dibromo-2,5-bis(dodecyloxy)benzene (21): In an argon environment, to a stirred solution of 1,4-bis(dodecyloxy)benzene (6) $(500 \mathrm{mg}, 1.20 \mathrm{mmol})$ in dichloromethane $(20 \mathrm{~mL})$ was added dropwise a solution of bromine $(0.19 \mathrm{~mL}, 3.60 \mathrm{mmol})$ in dichloromethane over 10 minutes min at $0{ }^{\circ} \mathrm{C}$. The reaction was warmed to room temperature and the reaction was stirred at room 
temperature for overnight. The reaction mixture (an orange suspension) was quenched with sodium thiosulphate. The organic layers were extracted with dichloromethane and dried over $\mathrm{MgSO}_{4}$. After evaporation of the solvent, precipitated into $100 \mathrm{~mL}$ methanol to give $610 \mathrm{mg}$ (89\%) product as a white solid. ${ }^{1} \mathrm{H} \mathrm{NMR}\left(400 \mathrm{MHz}, \mathrm{CDCl}_{3}\right) \delta 7.08(\mathrm{~s}, 2 \mathrm{H}), 3.94(\mathrm{t}, J=6.5 \mathrm{~Hz}, 4 \mathrm{H}), 1.84-1.75$ $(\mathrm{m}, 4 \mathrm{H}), 1.46(\mathrm{~m}, J=15.3,7.2 \mathrm{~Hz}, 4 \mathrm{H}), 1.35-1.22(\mathrm{~m}, 30 \mathrm{H}), 0.88(\mathrm{t}, J=6.9 \mathrm{~Hz}, 6 \mathrm{H})$.

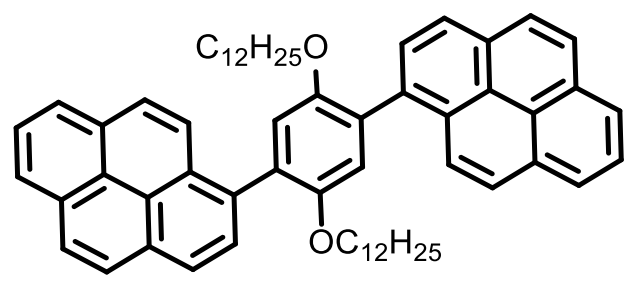

1,1'-(2,5-bis(dodecyloxy)-1,4-phenylene)dipyrene (5): In a glove box, 1,4-dibromo-2,5bis(dodecyloxy)benzene (7) (100 mg, $0.17 \mathrm{mmol}$ ), 4,4,5,5-tetramethyl-2-(1-pyrenyl)-1,3,2dioxaborolane $(135 \mathrm{mg}, 0.41 \mathrm{mmol}), \mathrm{Pd}\left(\mathrm{PPh}_{3}\right)_{4}(11.48 \mathrm{mg}, 0.010 \mathrm{mmol})$ and potassium carbonate $(115 \mathrm{mg}, 0.83 \mathrm{mmol})$ were added in dry THF $(8 \mathrm{~mL})$ and deionized water $(2.0 \mathrm{~mL})$ was added outside the glove box. The resulting mixture was refluxed at $80^{\circ} \mathrm{C}$ for overnight. Upon completion, the reaction was diluted with water, extracted with dichloromethane $(10 \mathrm{~mL} \times 3)$, dried on $\mathrm{MgSO}_{4}$. After evaporation of the solvent, the residue was purified by flash column chromatography with hexane and 0 to $30 \%$ dichloromethane to give $110 \mathrm{mg}(79 \%)$ of $\mathbf{8}$ as yellowish solid. ${ }^{1} \mathrm{H} \mathrm{NMR}$ $\left(400 \mathrm{MHz}, \mathrm{CDCl}_{3}\right) \delta 8.32-8.26(\mathrm{dd}, 2 \mathrm{H}), 8.22(\mathrm{~m}, J=7.8,2.9 \mathrm{~Hz}, 4 \mathrm{H}), 8.17(\mathrm{~d}, J=6.1 \mathrm{~Hz}, 2 \mathrm{H})$, $8.16-8.12(\mathrm{~m}, 6 \mathrm{H}), 8.10(\mathrm{t}, J=1.7 \mathrm{~Hz}, 1 \mathrm{H}), 8.04(\mathrm{~m}, J=12.1,8.4,6.2 \mathrm{~Hz}, 3 \mathrm{H}), 7.22(\mathrm{~s}, 1 \mathrm{H}), 7.21$ $(\mathrm{s}, 1 \mathrm{H}), 3.89-3.79(\mathrm{~m}, 4 \mathrm{H}), 1.36(\mathrm{~m}, 4 \mathrm{H}), 1.30-1.15(\mathrm{~m}, 14 \mathrm{H}), 1.14-0.95(\mathrm{~m}, 10 \mathrm{H}), 0.87(\mathrm{~m}$, $J=7.1 \mathrm{~Hz}, 18 \mathrm{H}) .{ }^{13} \mathrm{C} \mathrm{NMR}\left(101 \mathrm{MHz}, \mathrm{CDCl}_{3}\right) \delta 150.92,134.48,134.46,131.46,131.45,131.12$, $131.10,130.79$, 130.77, 130.67, 129.37, 129.34, 128.30, 128.28, 127.49, 127.30, 127.12, 127.05, 126.32, 125.86, 124.99, 124.97, 124.93, 124.92, 124.88, 124.84, 124.43, 118.07, 118.04, 69.82, $69.80,31.93,29.55,29.55,29.36,29.35,29.32$, 29.16, 29.06, 25.80, 22.71, 14.16. HRMS (TOFEI, m/z) calcd. for $\mathrm{C}_{62} \mathrm{H}_{70} \mathrm{O}_{2}[\mathrm{M}]+:$ 846.5376; found: 846.5389 .

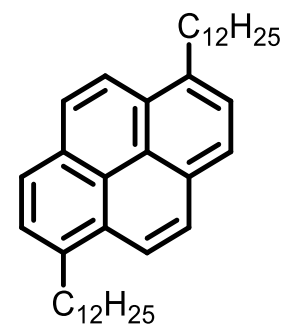

1,6-bis(dodecane)pyrene (22): In a glove box, 1-bromododecane (17) (1.00 g, $4.01 \mathrm{mmol}$ ) and magnesium (106 mg, $4.41 \mathrm{mmol})$, THF $(6 \mathrm{~mL})$ and diethyl ether $(4 \mathrm{~mL})$ were added into a seal tube. The reaction was stirred at $60{ }^{0} \mathrm{C}$ for $36 \mathrm{~h}$. After cooling room temperature, 1,6dibromopyrene (360 mg, $1.00 \mathrm{mmol})$ and $\mathrm{Ni}(\mathrm{dppp}) \mathrm{Cl}_{2}(22 \mathrm{mg}, 0.041 \mathrm{mmol})$ was added and the mixture was further stirred $60{ }^{\circ} \mathrm{C}$ for overnight. After cooling to room temperature, the organic layers were extracted with dichloromethane and dried over $\mathrm{MgSO}_{4}$. After evaporation of the solvent, the residue was purified by flash column chromatography with hexane to give $390 \mathrm{mg}$ $(72 \%)$ of 9 as off white solid. ${ }^{1} \mathrm{H} \mathrm{NMR}\left(400 \mathrm{MHz}, \mathrm{CDCl}_{3}\right): \delta 8.21(\mathrm{~d}, J=9.2 \mathrm{~Hz}, 2 \mathrm{H}), 8.08(\mathrm{~d}, J$ 
$=5.9 \mathrm{~Hz}, 2 \mathrm{H}), 8.04(\mathrm{~d}, J=9.3 \mathrm{~Hz}, 2 \mathrm{H}), 7.84(\mathrm{~d}, J=7.8 \mathrm{~Hz}, 2 \mathrm{H}), 3.36-3.27(\mathrm{~m}, 4 \mathrm{H}), 1.84(\mathrm{~m}, J$ $=15.3,7.7 \mathrm{~Hz}, 4 \mathrm{H}), 1.54-1.41(\mathrm{~m}, 4 \mathrm{H}), 1.35-1.19(\mathrm{~m}, 32 \mathrm{H}), 0.88(\mathrm{t}, J=8.7,5.1 \mathrm{~Hz}, 6 \mathrm{H}) .{ }^{13} \mathrm{C}$ NMR $\left(101 \mathrm{MHz}, \mathrm{CDCl}_{3}\right) \delta 137.03,129.48,128.86,127.20,127.15,125.49,124.38,122.54,33.75$, 31.93, 31.91, 29.85, 29.72, 29.69, 29.65, 29.62, 29.38, 29.37, 22.70, 14.13. HRMS (TOF-EI, m/z) calcd. for $\mathrm{C}_{40} \mathrm{H}_{58}[\mathrm{M}]+:$ 538.4539; found: 538.4543 .

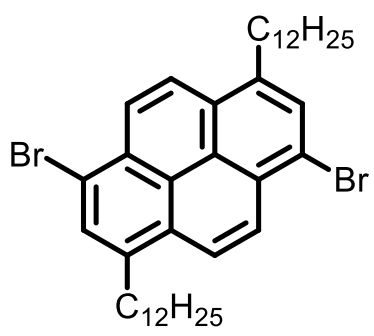

1,6-dibromo-3,8-didodecylpyrene (14): To the solution of 1,6-didodecylpyrene (9) (200 mg, $0.37 \mathrm{mmol})$ in $\mathrm{CHCl}_{3}(10 \mathrm{~mL})$ and DMF $(10 \mathrm{~mL})$, NBS $(145 \mathrm{mg}, 0.81 \mathrm{mmol})$ was added in oven dried Schlenk flask under argon atmosphere and the resulting reaction mixture was allowed to stir at room temperature for overnight. Then the reaction mixture quenched with methanol $(50 \mathrm{~mL})$. The precipitate was filtered and washed with copious amount of acetone to give $\mathbf{1 0}(216 \mathrm{mg}, 84 \%)$. ${ }^{1} \mathrm{H} \mathrm{NMR}\left(\mathrm{CDCl}_{3}, 400 \mathrm{MHz}\right): \delta 8.46(\mathrm{~d}, J=9.5 \mathrm{~Hz}, 2 \mathrm{H}), 8.27(\mathrm{~d}, J=9.5 \mathrm{~Hz}, 2 \mathrm{H}), 8.14(\mathrm{~s}, 2 \mathrm{H})$, $3.33-3.27(\mathrm{t}, 4 \mathrm{H}), 1.86(\mathrm{~m}, J=15.3,7.6 \mathrm{~Hz}, 4 \mathrm{H}), 1.51(\mathrm{~m}, J=14.8,6.9 \mathrm{~Hz}, 4 \mathrm{H}), 1.28(\mathrm{~m}, 32 \mathrm{H})$, $0.89(\mathrm{t}, J=6.9 \mathrm{~Hz}, 6 \mathrm{H}) .{ }^{13} \mathrm{C}$ NMR $\left(101 \mathrm{MHz}, \mathrm{CDCl}_{3}\right) \delta 138.55,131.69,128.36,128.01,126.19$, 126.02, 123.89, 120.31, 33.44, 31.90, 31.71, 29.74, 29.64, 29.61, 29.57, 29.51, 29.33, 22.67, 14.10 . HRMS (TOF-EI, m/z) calcd. for $\mathrm{C}_{40} \mathrm{H}_{56} \mathrm{Br}_{2}$ [M]+: 694.2749; found: 694.2762 .

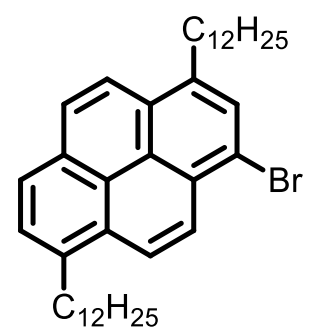

3-bromo-1,6-didodecylpyrene (7): To the solution of 1,6-didodecylpyrene (9) (200 mg, 0.37 $\mathrm{mmol})$ and $\mathrm{NH}_{4} \mathrm{NO}_{3}(9 \mathrm{mg}, 0.11 \mathrm{mmol})$ in $\mathrm{CHCl}_{3}(10 \mathrm{~mL})$, NBS $(66 \mathrm{mg}, 0.37 \mathrm{mmol})$ was added in oven dried Schlenk flask under argon atmosphere and the resulting reaction mixture was allowed to stir at room temperature for overnight. Then the reaction mixture quenched with water $(20 \mathrm{~mL})$ and extracted with DCM $(3 \times 20 \mathrm{~mL})$. The combined organic layers were dried over $\mathrm{MgSO}_{4}$, filtered and evaporated under reduced pressure to give $11\left(190 \mathrm{mg}(83 \%) .{ }^{1} \mathrm{H} \mathrm{NMR}\left(\mathrm{CDCl}_{3}, 400\right.\right.$ MHz): $\delta 8.42(\mathrm{~d}, J=9.5 \mathrm{~Hz}, 1 \mathrm{H}), 8.31(\mathrm{~d}, J=9.5 \mathrm{~Hz}, 1 \mathrm{H}), 8.17-8.04(\mathrm{~m}, 4 \mathrm{H}), 7.87(\mathrm{~d}, J=7.8$ $\mathrm{Hz}, 1 \mathrm{H}), 3.36-3.24$ (tt, 4H), $1.84(\mathrm{~m}, J=14.9,7.5 \mathrm{~Hz}, 4 \mathrm{H}), 1.48(\mathrm{~m}, J=13.7,6.6 \mathrm{~Hz}, 4 \mathrm{H}), 1.34$ - 1.19 (m, 32H), 0.88 (t, $J=13.1,6.9 \mathrm{~Hz}, 6 \mathrm{H}) .{ }^{13} \mathrm{C} \mathrm{NMR}\left(101 \mathrm{MHz}, \mathrm{CDCl}_{3}\right) \delta$ 137.92, 137.77, 130.97, 129.56, 128.67, 128.49, 127.85, 127.77, 127.50, 126.77, 125.64, 125.17, 124.87, 124.12, 
122.31, 119.42, 33.72, 33.43, 31.95, 31.91, 31.65, 29.80, 29.78, 29.66, 29.63, 29.60, 29.57, 29.54, 29.34, 22.68, 14.11. HRMS (TOF-EI, m/z) calcd. for $\mathrm{C}_{40} \mathrm{H}_{57} \mathrm{Br}$ [M]+: 616.3644; found: 616.3657.

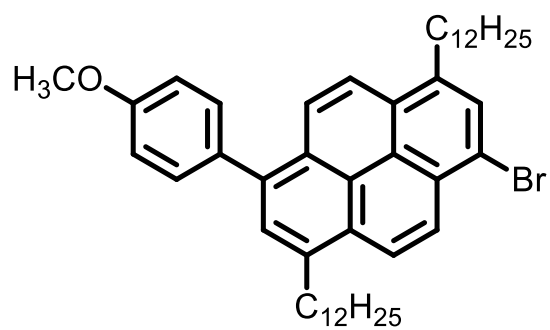

1-bromo-3,8-didodecyl-6-(4-methoxyphenyl)pyrene (8): In a glove box, 1,6-dibromo-3,8didodecylpyrene (10) (100 mg, $0.14 \mathrm{mmol}$ ), 4-methoxyphenylboronic acid (21.8 mg, $0.14 \mathrm{mmol}$ ), $\mathrm{Pd}\left(\mathrm{PPh}_{3}\right)_{4}(9.95 \mathrm{mg}, 0.0086 \mathrm{mmol})$ and potassium carbonate $(100 \mathrm{mg}, 0.72 \mathrm{mmol})$ were added in dry THF $(5 \mathrm{~mL})$ and deionized water $(1 \mathrm{~mL})$ was added outside the glove box. The resulting mixture was refluxed at $80{ }^{\circ} \mathrm{C}$ for overnight. Upon completion, the reaction was diluted with water, extracted 3 times with $\mathrm{DCM}$, dried on $\mathrm{MgSO}_{4}$. After evaporation of the solvent, the residue was purified by flash column chromatography with hexane and 0 to 30\% dichloromethane to give 69 $\mathrm{mg}(67 \%)$ of 12 as yellowish solid. ${ }^{1} \mathrm{H}$ NMR $\left(400 \mathrm{MHz}, \mathrm{CDCl}_{3}\right): \delta 8.43(\mathrm{~d}, J=9.5 \mathrm{~Hz}, 1 \mathrm{H}), 8.33$ $(\mathrm{d}, J=9.5 \mathrm{~Hz}, 1 \mathrm{H}), 8.18(\mathrm{~d}, J=7.5 \mathrm{~Hz}, 1 \mathrm{H}), 8.09$ (d, $J=9.8 \mathrm{~Hz}, 2 \mathrm{H}), 7.85(\mathrm{~s}, 1 \mathrm{H}), 7.60-7.50$ (d, $2 \mathrm{H}), 7.14-7.06(\mathrm{~d}, 2 \mathrm{H}), 3.94(\mathrm{~s}, 3 \mathrm{H}), 3.38-3.31(\mathrm{t}, 2 \mathrm{H}), 3.28-3.21(\mathrm{t}, 2 \mathrm{H}), 1.84(\mathrm{~m}, J=15.4$, $7.8 \mathrm{~Hz}, 4 \mathrm{H}), 1.47(\mathrm{~m}, J=22.7,7.5 \mathrm{~Hz}, 4 \mathrm{H}), 1.25(\mathrm{~m}, 32 \mathrm{H}), 0.87(\mathrm{t}, J=6.8 \mathrm{~Hz}, 6 \mathrm{H}) .{ }^{13} \mathrm{C} \mathrm{NMR}$ $\left(100 \mathrm{MHz}, \mathrm{CDCl}_{3}\right): \delta 158.99,137.64,137.49,137.25,133.53,131.63,131.13,129.62,128.35$, $128.17,127.96,127.08,125.52,125.42,125.34,124.10,122.24,119.41,113.80,55.42,33.79$, $33.44,31.95,31.91,31.65,29.87,29.77,29.66,29.65,29.63,29.62,29.59,29.57,29.53,29.34$, 22.68, 14.12. HRMS (TOF-EI, m/z) calcd. for $\mathrm{C}_{47} \mathrm{H}_{63} \mathrm{BrO}[\mathrm{M}]+$ : 722.4062 ; found: 722.4076 .

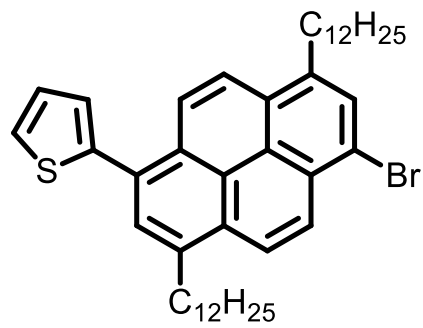

2-(6-bromo-3,8-didodecylpyren-1-yl)thiophene (9): In a glove box, 1,6-dibromo-3,8didodecylpyrene (10) (100 mg, $0.14 \mathrm{mmol}), 2$-(tributylstannyl)thiophene $(53.5 \mathrm{mg}, 0.14 \mathrm{mmol})$ and $\mathrm{Pd}\left(\mathrm{PPh}_{3}\right)_{4}(9.95 \mathrm{mg}, 0.0086 \mathrm{mmol})$ were added in dry toluene $(5 \mathrm{~mL})$. The resulting mixture was refluxed at $110{ }^{\circ} \mathrm{C}$ for overnight. Upon completion, the reaction was diluted with water, extracted 3 times with DCM, dried on $\mathrm{MgSO}_{4}$. After evaporation of the solvent, the residue was purified by flash column chromatography with hexane and 0 to $10 \%$ dichloromethane to give 44 $\mathrm{mg}(43 \%)$ of $\mathbf{1 3}$ as yellowish solid. ${ }^{1} \mathrm{H}$ NMR $(400 \mathrm{MHz}, \mathrm{CDCl} 3): \delta 8.47(\mathrm{~d}, J=9.5 \mathrm{~Hz}, 1 \mathrm{H}), 8.22$ $(\mathrm{d}, J=10.2 \mathrm{~Hz}, 2 \mathrm{H}), 8.08(\mathrm{dd}, J=11.6,8.5 \mathrm{~Hz}, 2 \mathrm{H}), 7.95(\mathrm{~s}, 1 \mathrm{H}), 7.86(\mathrm{~d}, J=7.8 \mathrm{~Hz}, 1 \mathrm{H}), 7.51$ $(\mathrm{d}, J=5.1 \mathrm{~Hz}, 1 \mathrm{H}), 7.38(\mathrm{~d}, J=3.5 \mathrm{~Hz}, 1 \mathrm{H}), 3.32(\mathrm{q}, J=15.2,7.2 \mathrm{~Hz}, 4 \mathrm{H}), 1.85(\mathrm{~m}, J=15.0,7.6$ 
$\mathrm{Hz}, 4 \mathrm{H}), 1.57-1.43(\mathrm{~m}, 4 \mathrm{H}), 1.26(\mathrm{~m}, 32 \mathrm{H}), 0.88(\mathrm{t}, J=6.7 \mathrm{~Hz}, 6 \mathrm{H}) .{ }^{13} \mathrm{C} \mathrm{NMR}\left(100 \mathrm{MHz}, \mathrm{CDCl}_{3}\right)$ : $\delta 142.43,138.02,137.19,131.31,130.31,129.81,128.66,128.30,128.13,128.03,128.02,127.60$, $127.43,126.89,126.18,126.05,125.36,125.03,124.01,122.81,119.78,33.72,33.45,31.92,31.70$, 29.66, 29.65, 29.63, 29.59, 29.35, 22.69, 14.13. HRMS (TOF-EI, m/z) calcd. for $\mathrm{C}_{44} \mathrm{H}_{59} \mathrm{BrS}[\mathrm{M}]+$ : 698.3521; found: 698.3537 .

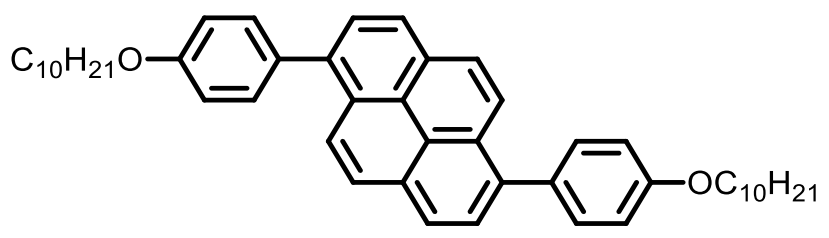

1,6-bis(4-(decyloxy)phenyl)pyrene (23): In a glove box, 1,6-dibromopyrene (100 $\mathrm{mg}, 0.28$ mmol), 2-(4-(decyloxy)phenyl)-4,4,5,5-tetramethyl-1,3,2-dioxaborolane (3) (220.2 mg, 0.61 $\mathrm{mmol}), \mathrm{Pd}\left(\mathrm{PPh}_{3}\right)_{4}(19.25 \mathrm{mg}, 0.017 \mathrm{mmol})$ and potassium carbonate $(193 \mathrm{mg}, 1.39 \mathrm{mmol})$ were added in dry THF $(8 \mathrm{~mL})$ and deionized water $(1.6 \mathrm{~mL})$ was added outside the glove box. The resulting mixture was refluxed at $80{ }^{\circ} \mathrm{C}$ for overnight. Upon completion, the reaction was diluted with water, extracted 3 times with $\mathrm{DCM}$, dried on $\mathrm{MgSO}_{4}$. After evaporation of the solvent, the residue was purified by flash column chromatography with hexane and 0 to $30 \%$ dichloromethane to give $91 \mathrm{mg}(49 \%)$ of $\mathbf{1 4}$ as yellowish solid. ${ }^{1} \mathrm{H}$ NMR (400 MHz, $\left.\mathrm{CDCl}_{3}\right) \delta 8.19$ (dd, $J=11.2$, $8.6 \mathrm{~Hz}, 4 \mathrm{H}), 7.99$ (dd, $J=24.0,8.5 \mathrm{~Hz}, 4 \mathrm{H}), 7.56(4, J=8.5 \mathrm{~Hz}, 1 \mathrm{H}), 7.10(\mathrm{~d}, J=8.5 \mathrm{~Hz}, 4 \mathrm{H})$, $4.09(\mathrm{t}, J=6.6 \mathrm{~Hz}, 4 \mathrm{H}), 1.91-1.83(\mathrm{~m}, J=14.8,6.9 \mathrm{~Hz}, 4 \mathrm{H}), 1.48-1.19(\mathrm{~m}, 28 \mathrm{H}), 0.90(\mathrm{t}, J=$ $6.8 \mathrm{~Hz}, 6 \mathrm{H}) .{ }^{13} \mathrm{C} \mathrm{NMR}\left(101 \mathrm{MHz}, \mathrm{CDCl}_{3}\right) \delta 158.57,137.53,133.43,131.60,130.19,128.95$, $127.80,127.29,125.30,125.22,124.34,114.40,68.16,31.93,29.63,29.60,29.46,29.38,29.35$, 26.14, 22.70, 14.14. HRMS (TOF-EI, m/z) calcd. for $\mathrm{C}_{48} \mathrm{H}_{58} \mathrm{O}_{2}$ [M]+: 666.4437; found: 666.4423.

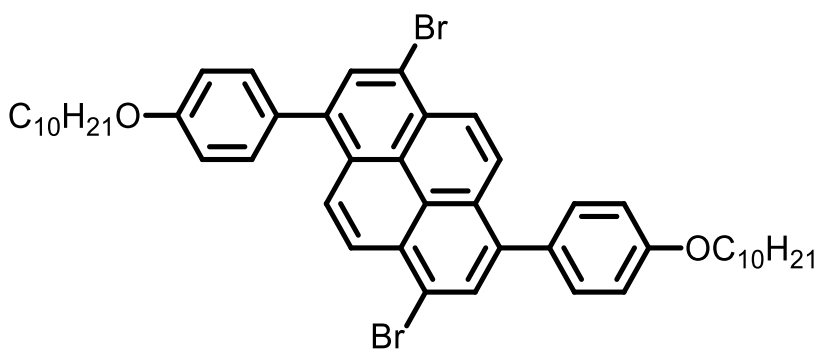

1,6-dibromo-3,8-bis(4-(decyloxy)phenyl)pyrene (15): To the solution of 1,6-bis(4(decyloxy)phenyl)pyrene (14) $80 \mathrm{mg}, 0.12 \mathrm{mmol}$ ) in $\mathrm{CHCl}_{3}(5 \mathrm{~mL})$ and DMF (5 mL), NBS (53 $\mathrm{mg}, 0.30 \mathrm{mmol}$ ) was added in oven dried Schlenk flask under argon atmosphere and the resulting reaction mixture was allowed to stir at room temperature for overnight. Then the reaction mixture quenched with methanol $(50 \mathrm{~mL})$. The precipitate was filtered and washed with copious amount of acetone to give $15(71 \mathrm{mg}, 72 \%) .{ }^{1} \mathrm{H}$ NMR $\left(\mathrm{CDCl}_{3}, 400 \mathrm{MHz}\right): \delta 8.39(\mathrm{~d}, J=9.5 \mathrm{~Hz}, 2 \mathrm{H}), 8.26$ (s, 2H), $8.24-8.21(\mathrm{~d}, 2 \mathrm{H}), 7.54-7.48(\mathrm{~d}, 4 \mathrm{H}), 7.12-7.06(\mathrm{~d}, 4 \mathrm{H}), 4.08(\mathrm{t}, J=6.6 \mathrm{~Hz}, 4 \mathrm{H}), 1.92$ $-1.83(\mathrm{~m}, 4 \mathrm{H}), 1.53(\mathrm{~m}, J=15.2,7.4 \mathrm{~Hz}, 4 \mathrm{H}), 1.41-1.26(\mathrm{~m}, 24 \mathrm{H}), 0.91(\mathrm{t}, J=6.9 \mathrm{~Hz}, 6 \mathrm{H}) .{ }^{13} \mathrm{C}$ NMR (100 MHz, $\left.\mathrm{CDCl}_{3}\right): \delta 158.98,138.72,132.26,131.65,131.57,128.66,128.40,126.61$, 126.06, 126.01, 120.20, 114.57, 68.20, 31.92, 29.63, 29.60, 29.45, 29.35, 29.33, 26.12, 22.71, 14.14. HRMS (TOF-EI, m/z) calcd. for $\mathrm{C}_{48} \mathrm{H}_{56} \mathrm{Br}_{2} \mathrm{O}_{2}$ [M]+: 822.2647; found: 822.2667. 


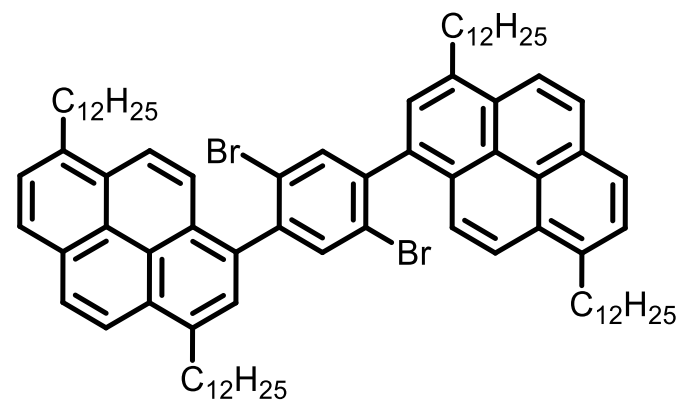

3,3'-(2,5-dibromo-1,4-phenylene)bis(1,6-didodecylpyrene) (11): In a glove box, 3-bromo-1,6didodecylpyrene (11) $(50 \mathrm{mg}, 0.081 \mathrm{mmol}$ ), 2,2'-(2,5-dibromo-1,4-phenylene)bis(4,4,5,5tetramethyl-1,3,2-dioxaborolane) (5) (19.7 mg, $0.040 \mathrm{mmol}), \mathrm{Pd}\left(\mathrm{PPh}_{3}\right)_{4}(2.80 \mathrm{mg}, 0.0024 \mathrm{mmol})$, potassium carbonate $(28.06 \mathrm{mg}, 0.20 \mathrm{mmol})$ and 1 drop of aliquot 336 were added in dry toluene $(1.8 \mathrm{~mL})$ and deionized water $(0.60 \mathrm{~mL})$ was added outside the glove box. The resulting mixture was refluxed at $110{ }^{\circ} \mathrm{C}$ for overnight. Upon completion, the reaction was diluted with water, extracted 3 times with DCM, dried on $\mathrm{MgSO}_{4}$. After evaporation of the solvent, the residue was purified by flash column chromatography with hexane and 0 to $20 \%$ dichloromethane to give 33 mg (32\%) of 16 as yellowish solid. ${ }^{1} \mathrm{H}$ NMR $\left(400 \mathrm{MHz}, \mathrm{CDCl}_{3}\right)$ : $\delta 8.37-8.26(\mathrm{~m}, 4 \mathrm{H}), 8.18-$ $8.11(\mathrm{~m}, 4 \mathrm{H}), 8.01-7.86(\mathrm{~m}, 8 \mathrm{H}), 3.50-3.28(\mathrm{~m}, 8 \mathrm{H}), 2.00-1.83(\mathrm{~m}, 8 \mathrm{H}), 1.58-1.47(\mathrm{~m}, 8 \mathrm{H})$, $1.36-1.19(\mathrm{~m}, 64 \mathrm{H}), 0.94-0.82(\mathrm{~m}, 12 \mathrm{H}) .{ }^{13} \mathrm{C} \mathrm{NMR}\left(100 \mathrm{MHz}, \mathrm{CDCl}_{3}\right): \delta$ 142.95, 137.55, $137.53,136.48,136.47,135.93,134.07,129.71,129.22,128.68,128.66,128.59,128.58,127.76$, $127.51,127.48,127.15,127.13,125.62,124.99,124.98,124.64,124.61,123.38,123.36,123.28$, $123.19,122.49,33.84,33.79,33.73,32.02,31.98,31.89,31.79,31.72,29.91,29.87,29.86,29.80$, 29.66, 29.63, 29.61, 29.60, 29.33, 29.32, 22.66, 14.08. HRMS (TOF-EI, m/z) calcd. for $\mathrm{C}_{86} \mathrm{H}_{116} \mathrm{Br}_{2}$ [M]+: 1306.7522; found: 1306.7491 .

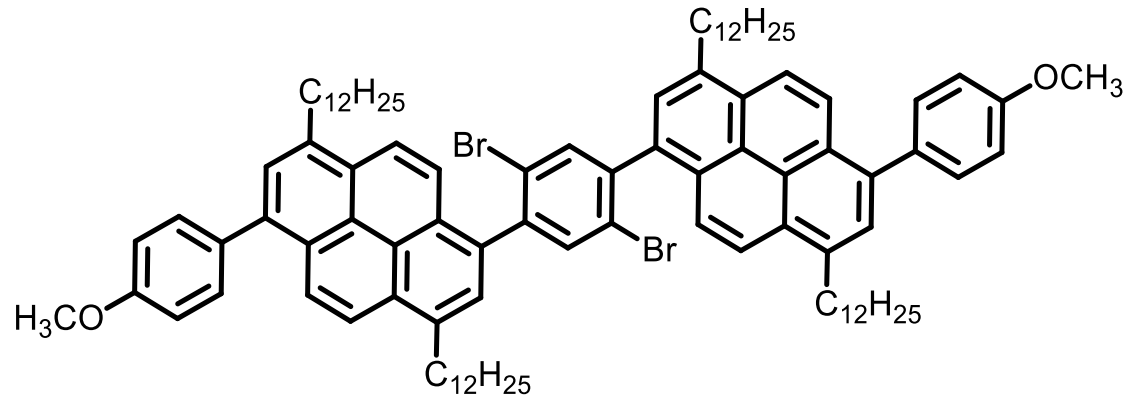

8,8'-(2,5-dibromo-1,4-phenylene)bis(1,6-didodecyl-3-(4-methoxyphenyl)pyrene) (12): In a glove box, 1-bromo-3,8-didodecyl-6-(4-methoxyphenyl)pyrene (12) $(50 \mathrm{mg}, 0.069 \mathrm{mmol}), 2,2$ '(2,5-dibromo-1,4-phenylene)bis(4,4,5,5-tetramethyl-1,3,2-dioxaborolane) (5) (16.8 mg, 0.035 $\mathrm{mmol}), \mathrm{Pd}\left(\mathrm{PPh}_{3}\right)_{4}(2.40 \mathrm{mg}, 0.0021 \mathrm{mmol})$, potassium carbonate $(23.9 \mathrm{mg}, 0.17 \mathrm{mmol})$ and 1 drop of aliquot 336 were added in dry toluene $(1.8 \mathrm{~mL})$ and deionized water $(0.60 \mathrm{~mL})$ was added outside the glove box. The resulting mixture was refluxed at $110{ }^{\circ} \mathrm{C}$ for overnight. Upon 
completion, the reaction was diluted with water, extracted 3 times with DCM, dried on $\mathrm{MgSO}_{4}$. After evaporation of the solvent, the residue was purified by flash column chromatography with hexane and 0 to $30 \%$ dichloromethane to give $36 \mathrm{mg}(35 \%)$ of $\mathbf{1 7}$ as yellowish solid. ${ }^{1} \mathrm{H}$ NMR $\left(400 \mathrm{MHz}, \mathrm{CDCl}_{3}\right): \delta 8.39-8.22(\mathrm{~m}, 6 \mathrm{H}), 8.01-7.85(\mathrm{~m}, 8 \mathrm{H}), 7.64-7.55(\mathrm{~m}, 4 \mathrm{H}), 7.17-7.10$ $(\mathrm{m}, 4 \mathrm{H}), 4.01-3.89(\mathrm{~s}, 6 \mathrm{H}), 3.48-3.27(\mathrm{~m}, 8 \mathrm{H}), 1.92(\mathrm{~m}, J=17.6,8.7 \mathrm{~Hz}, 8 \mathrm{H}), 1.63-1.45(\mathrm{~m}$, $8 \mathrm{H}), 1.36-1.18(\mathrm{~m}, 64 \mathrm{H}), 0.96-0.80(\mathrm{~m}, 12 \mathrm{H}) .{ }^{13} \mathrm{C} \mathrm{NMR}\left(100 \mathrm{MHz}, \mathrm{CDCl}_{3}\right): \delta 158.96,143.03$, 137.30, 137.27, 137.06, 137.03, 136.23, 136.22, 135.89, 134.10, 133.78, 131.69, 129.36, 129.03, $128.66,127.95,127.92$, 127.49, 127.46, 127.19, 127.18, 126.02, 126.01, 125.88, 125.66, 123.47, $123.45,123.24,123.15,122.45,113.81,55.44,31.92,29.69,29.65,29.62,29.36,22.69,14.13$. HRMS (TOF-EI, m/z) calcd. for $\mathrm{C}_{100} \mathrm{H}_{128} \mathrm{Br}_{2} \mathrm{O}_{2}$ [M]+: 1518.8281 ; found: 1518.8276 .

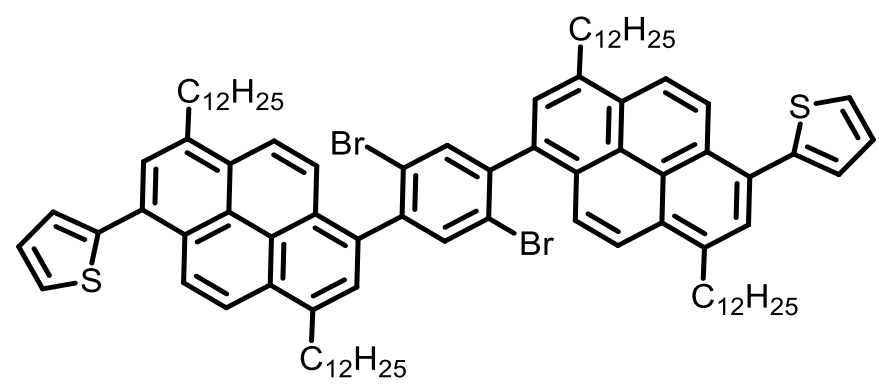

2,2'-((2,5-dibromo-1,4-phenylene)bis(3,8-didodecylpyrene-6,1-diyl))dithiophene (13): In a glove box, 2-(6-bromo-3,8-didodecylpyren-1-yl)thiophene (13) (40 mg, 0.057 mmol), 2,2'-(2,5dibromo-1,4-phenylene)bis(4,4,5,5-tetramethyl-1,3,2-dioxaborolane) (5) (13.9 mg, $0.029 \mathrm{mmol})$, $\mathrm{Pd}\left(\mathrm{PPh}_{3}\right)_{4}(1.98 \mathrm{mg}, 0.0017 \mathrm{mmol})$, potassium carbonate $(19.8 \mathrm{mg}, 0.14 \mathrm{mmol})$ and 1 drop of aliquot 336 were added in dry toluene $(1.5 \mathrm{~mL})$ and deionized water $(0.50 \mathrm{~mL})$ was added outside the glove box. The resulting mixture was refluxed at $110{ }^{\circ} \mathrm{C}$ for overnight. Upon completion, the reaction was diluted with water, extracted 3 times with $\mathrm{DCM}$, dried on $\mathrm{MgSO}_{4}$. After evaporation of the solvent, the residue was purified by flash column chromatography with hexane and 0 to $20 \%$ dichloromethane to give $24 \mathrm{mg}(29 \%)$ of 18 as yellowish solid. ${ }^{1} \mathrm{H}$ NMR (400 $\left.\mathrm{MHz}, \mathrm{CDCl}_{3}\right): \delta$ $8.62-8.53(\mathrm{~d}, 2 \mathrm{H}), 8.36-8.26(\mathrm{~m}, 4 \mathrm{H}), 8.04-7.92(\mathrm{~m}, 8 \mathrm{H}), 7.54(\mathrm{~m}, J=5.3,3.2,1.1 \mathrm{~Hz}, \mathrm{mH})$, $7.41(\mathrm{~m}, J=3.4,1.2 \mathrm{~Hz}, 2 \mathrm{H}), 7.33-7.27(\mathrm{~m}, 2 \mathrm{H}), 3.40(\mathrm{~m}, J=19.3,11.4 \mathrm{~Hz}, 8 \mathrm{H}), 2.01-1.83$ $(\mathrm{m}, 8 \mathrm{H}), 1.64-1.46(\mathrm{~m}, 8 \mathrm{H}), 1.24(\mathrm{~m}, J=19.6 \mathrm{~Hz}, 64 \mathrm{H}), 0.96-0.78(\mathrm{~m}, 12 \mathrm{H}) .{ }^{13} \mathrm{C}$ NMR $(100$ $\left.\mathrm{MHz}_{1} \mathrm{CDCl}_{3}\right): \delta 142.98,142.72,136.99,136.97,136.63,135.91,134.41,130.05,130.03,129.63$, 129.60, 129.01, 128.85, 128.67, 128.65, 127.97, 127.75, 127.73, 127.46, 127.42, 126.08, 125.74, 125.28, 125.03, 123.45, 123.43, 123.17, 123.08, 123.00, 33.80, 31.91, 29.68, 29.64, 29.61, 29.35, 22.68, 14.12. HRMS (TOF-EI, m/z) calcd. for $\mathrm{C}_{94} \mathrm{H}_{120} \mathrm{Br}_{2} \mathrm{~S}_{2}[\mathrm{M}]+:$ 1470.7193; found: 1470.7173 . 


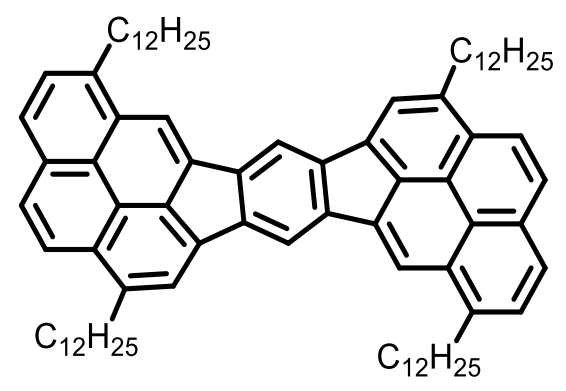

5,9,14,18-tetradodecyl-s-indaceno[1,2,3-cd:5,6,7-c'd']dipyrene (1a): In the glove box, 3,3'(2,5-dibromo-1,4-phenylene)bis(1,6-didodecylpyrene) (16) (20 mg, $0.015 \mathrm{mmol}), \mathrm{Pd}\left(\mathrm{PCy}_{3}\right)_{2} \mathrm{Cl}_{2}$ $(10.0 \mathrm{mg}, 0.014 \mathrm{mmol})$, DBU $(0.10 \mathrm{~mL})$, and DMA $(2 \mathrm{~mL})$ were added to a $10 \mathrm{~mL}$ seal tube. The seal tube was removed from the glove box and placed into a preheated oil bath where it was heated at $140{ }^{\circ} \mathrm{C}$ for overnight. After cooling, the reaction mixture was poured into $100 \mathrm{~mL}$ methanol, filtered, washed with copious amount of acetone and dried over high vacuum to give the product $15 \mathrm{mg}(86 \%)$ as brick red solid. ${ }^{1} \mathrm{H}$ NMR $\left(300 \mathrm{MHz}, \mathrm{C}_{2} \mathrm{D}_{2} \mathrm{Cl}_{4}, 80{ }^{\circ} \mathrm{C}\right): \delta 8.28(\mathrm{~s}, 2 \mathrm{H}), 8.13(\mathrm{~s}, 2 \mathrm{H})$, $7.77(\mathrm{~s}, 2 \mathrm{H}), 7.63(\mathrm{~d}, J=9.5 \mathrm{~Hz}, 2 \mathrm{H}), 7.54(\mathrm{~d}, J=7.7 \mathrm{~Hz}, 2 \mathrm{H}), 7.42(\mathrm{~d}, J=9.1 \mathrm{~Hz}, 2 \mathrm{H}), 7.29(\mathrm{~d}$, $J=7.6 \mathrm{~Hz}, 2 \mathrm{H}), 2.93(\mathrm{t}, J=7.4 \mathrm{~Hz}, 4 \mathrm{H}), 2.85(\mathrm{t}, J=7.6 \mathrm{~Hz}, 4 \mathrm{H}), 1.48-1.36(\mathrm{~m}, 8 \mathrm{H}), 1.00(\mathrm{~m}$, $8 \mathrm{H}), 0.71(\mathrm{~m}, J=25.7 \mathrm{~Hz}, 64 \mathrm{H}), 0.25(\mathrm{t}, J=6.2 \mathrm{~Hz}, 12 \mathrm{H})$. HRMS (TOF-EI, m/z) calcd. for $\mathrm{C}_{86} \mathrm{H}_{114}$ [M]+: 1146.8999; found: 1146.8976 .

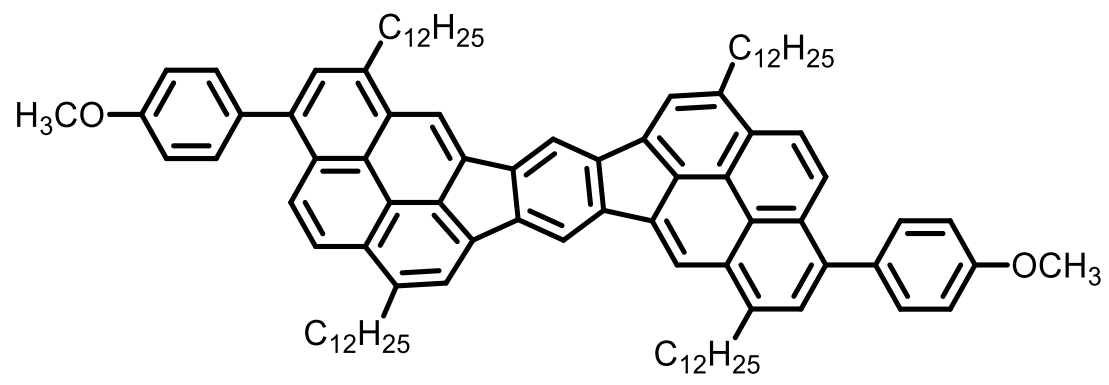

5,9,14,18-tetradodecyl-3,12-bis(4-methoxyphenyl)-s-indaceno[1,2,3-cd:5,6,7-c'd']dipyrene

(1b): In the glove box, 8,8'-(2,5-dibromo-1,4-phenylene)bis(1,6-didodecyl-3-(4methoxyphenyl)pyrene) (17) (20 mg, $0.013 \mathrm{mmol}), \mathrm{Pd}\left(\mathrm{PCy}_{3}\right)_{2} \mathrm{Cl}_{2}(8.70 \mathrm{mg}, 0.012 \mathrm{mmol})$, DBU $(0.10 \mathrm{~mL})$, and DMA $(2 \mathrm{~mL})$ were added to a $10 \mathrm{~mL}$ seal tube. The seal tube was removed from the glove box and placed into a preheated oil bath where it was heated at $140{ }^{\circ} \mathrm{C}$ for overnight. After cooling, the reaction mixture was poured into $100 \mathrm{~mL}$ methanol, filtered, washed with copious amount of acetone and dried over high vacuum to give the product $16 \mathrm{mg}(90 \%)$ as red solid. ${ }^{1} \mathrm{H}$ NMR (300MHz, $\left.\mathrm{C}_{2} \mathrm{D}_{2} \mathrm{Cl}_{4}, 60{ }^{0} \mathrm{C}\right) \delta 8.33(\mathrm{~s}, 2 \mathrm{H}), 8.18(\mathrm{~s}, 2 \mathrm{H}), 7.80(\mathrm{~s}, 2 \mathrm{H}), 7.57$ (q, $J=$ $9.3 \mathrm{~Hz}, 4 \mathrm{H}), 7.28(\mathrm{~s}, 2 \mathrm{H}), 7.01(\mathrm{~d}, J=8.2 \mathrm{~Hz}, 4 \mathrm{H}), 6.52(\mathrm{~d}, J=8.1 \mathrm{~Hz}, 4 \mathrm{H}), 3.40-3.28(\mathrm{~s}, 6 \mathrm{H})$, $2.96(\mathrm{t}, 4 \mathrm{H}), 2.83(\mathrm{t}, 4 \mathrm{H}), 1.42(\mathrm{~m}, J=27.4 \mathrm{~Hz}, 8 \mathrm{H}), 0.99(\mathrm{~m}, 8 \mathrm{H}), 0.67(\mathrm{~m}, 64 \mathrm{H}), 0.25(\mathrm{t}, J=5.2$ $\mathrm{Hz}, 12 \mathrm{H}$ ). HRMS (TOF-EI, m/z) calcd. for $\mathrm{C}_{100} \mathrm{H}_{126} \mathrm{O}_{2}$ [M]+: 1358.9758; found: 1358.9736. 


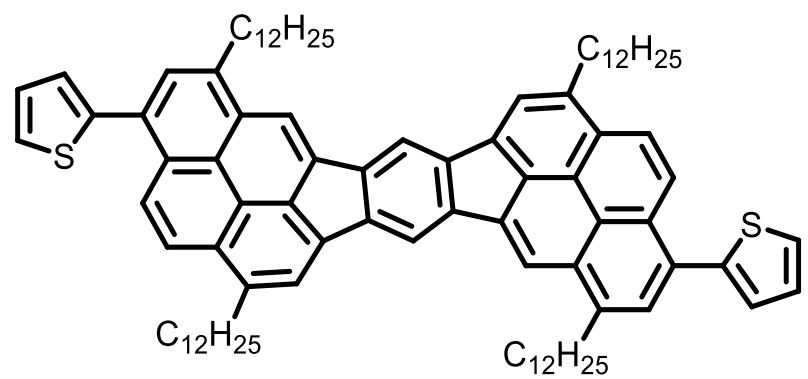

2,2'-(5,9,14,18-tetradodecyl-s-indaceno[1,2,3-cd:5,6,7-c'd']dipyrene-3,12-diyl)dithiophene (1c): In the glove box, 2,2'-((2,5-dibromo-1,4-phenylene)bis(3,8-didodecylpyrene-6,1diyl))dithiophene (18) (20 mg, $0.014 \mathrm{mmol}), \mathrm{Pd}\left(\mathrm{PCy}_{3}\right)_{2} \mathrm{Cl}_{2}$ (9 mg, $\left.0.012 \mathrm{mmol}\right), \mathrm{DBU}(0.10 \mathrm{~mL})$, and DMA $(2 \mathrm{~mL})$ were added to a $10 \mathrm{~mL}$ seal tube. The seal tube was removed from the glove box and placed into a preheated oil bath where it was heated at $140{ }^{\circ} \mathrm{C}$ for overnight. After cooling, the reaction mixture was poured into $100 \mathrm{~mL}$ methanol, filtered, washed with copious amount of acetone and dried over high vacuum to give the product $14 \mathrm{mg}(78 \%)$ as red solid. ${ }^{1} \mathrm{H}$ NMR $\left(300 \mathrm{MHz}, \mathrm{C}_{2} \mathrm{D}_{2} \mathrm{Cl}_{4}, 60{ }^{0} \mathrm{C}\right) \delta 8.23(\mathrm{~s}, 2 \mathrm{H}), 8.10(\mathrm{~s}, 2 \mathrm{H}), 7.83(\mathrm{~d}, J=9.6 \mathrm{~Hz}, 2 \mathrm{H}), 7.75(\mathrm{~s}, 2 \mathrm{H}), 7.62$ $(\mathrm{d}, J=9.7 \mathrm{~Hz}, 2 \mathrm{H}), 7.39(\mathrm{~s}, 2 \mathrm{H}), 6.94(\mathrm{~d}, J=5.1 \mathrm{~Hz}, 2 \mathrm{H}), 6.81(\mathrm{~d}, 2 \mathrm{H}), 6.67(\mathrm{t}, J=4.6 \mathrm{~Hz}, 2 \mathrm{H})$, $2.89(\mathrm{~d}, J=8.0 \mathrm{~Hz}, 2 \mathrm{H}), 2.82(\mathrm{~d}, J=5.9 \mathrm{~Hz}, 2 \mathrm{H}), 1.48-1.32(\mathrm{~m}, 5 \mathrm{H}), 1.06-0.94(\mathrm{~m}, 5 \mathrm{H}), 0.67$ $(\mathrm{s}, 41 \mathrm{H}), 0.26(\mathrm{~d}, J=5.1 \mathrm{~Hz}, 6 \mathrm{H})$. HRMS (TOF-EI, m/z) calcd. for $\mathrm{C}_{94} \mathrm{H}_{118} \mathrm{~S}_{2}[\mathrm{M}]+\mathrm{t}: 1310.8675$; found: 1310.8713 .

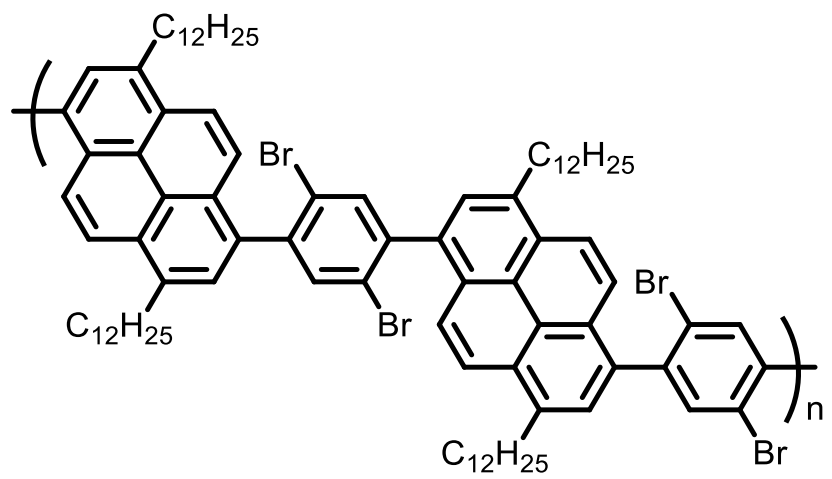

Poly[3-(2,5-dibromophenyl)-1,6-didodecylpyrene] (16): In a glove box, 1,6-dibromo-3,8didodecylpyrene (10) (50 mg, $0.072 \mathrm{mmol}), 2,2$-(2,5-dibromo-1,4-phenylene)bis(4,4,5,5tetramethyl-1,3,2-dioxaborolane) (5) $(35 \mathrm{mg}, 0.072 \mathrm{mmol}), \mathrm{Pd}\left(\mathrm{PPh}_{3}\right)_{4}(8.32 \mathrm{mg}, 0.0072 \mathrm{mmol})$, potassium carbonate $(50.04 \mathrm{mg}, 0.36 \mathrm{mmol})$ and 1 drop of aliquot 336 were added in dry toluene $(1.8 \mathrm{~mL})$ and deionized water $(0.60 \mathrm{~mL})$ was added outside the glove box. The resulting mixture was refluxed at $110^{\circ} \mathrm{C}$ for 3 days. Upon completion, the reaction was diluted with dichloromethane and water and extracted 3 times with DCM. After evaporation of the solvent, the reaction mixture was precipitated into $100 \mathrm{~mL}$ methanol, filtered, washed with copious amount of acetone and dried over high vacuum. Recycling preparative GPC was used to further purify the polymer to $14 \mathrm{mg}$ (25\%) as yellow solid. ${ }^{1} \mathrm{H}$ NMR $\left(400 \mathrm{MHz}, \mathrm{CDCl}_{3}\right) \delta 8.46-7.59(\mathrm{~m}, J=167.6 \mathrm{~Hz}, 16 \mathrm{H}), 3.66-$ $2.90(\mathrm{~m}, 8 \mathrm{H}), 2.07-1.71(\mathrm{~m}, 8 \mathrm{H}), 1.38-1.06(\mathrm{~m}, 72 \mathrm{H}), 0.90-0.76(\mathrm{~m}, 12 \mathrm{H})$. 


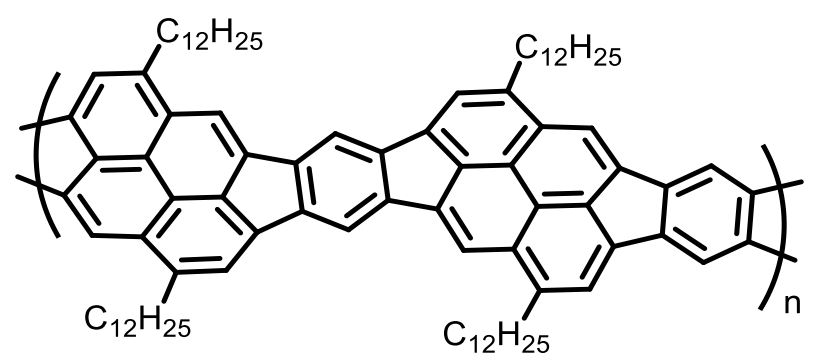

Poly[5,12-didodecylindeno[1,2,3-cd]pyrene] (2a): In the glove box, Poly[3-(2,5dibromophenyl)-1,6-didodecylpyrene] (19) (14 mg, $0.0089 \mathrm{mmol}), \mathrm{Pd}\left(\mathrm{PCy}_{3}\right)_{2} \mathrm{Cl}_{2}$ (6 mg, 0.0080 mmol), DBU $(0.10 \mathrm{~mL})$, and DMA $(2 \mathrm{~mL})$ were added to a $10 \mathrm{~mL}$ seal tube. The seal tube was removed from the glove box and placed into a preheated oil bath where it was heated at $140{ }^{\circ} \mathrm{C}$ for overnight. After cooling, the reaction mixture was poured into $100 \mathrm{~mL}$ methanol, filtered, washed with copious amount of acetone, dichloromethane and dried over high vacuum to give $8 \mathrm{mg}$ (70\%) as purple solid. We are unable to get molecular weight and NMR spectrum due to the insolubility of polymer in common organic solvent like as chloroform, dichloromethane, tetrahydrofuran, 1,1,2,2-dichloroethane.

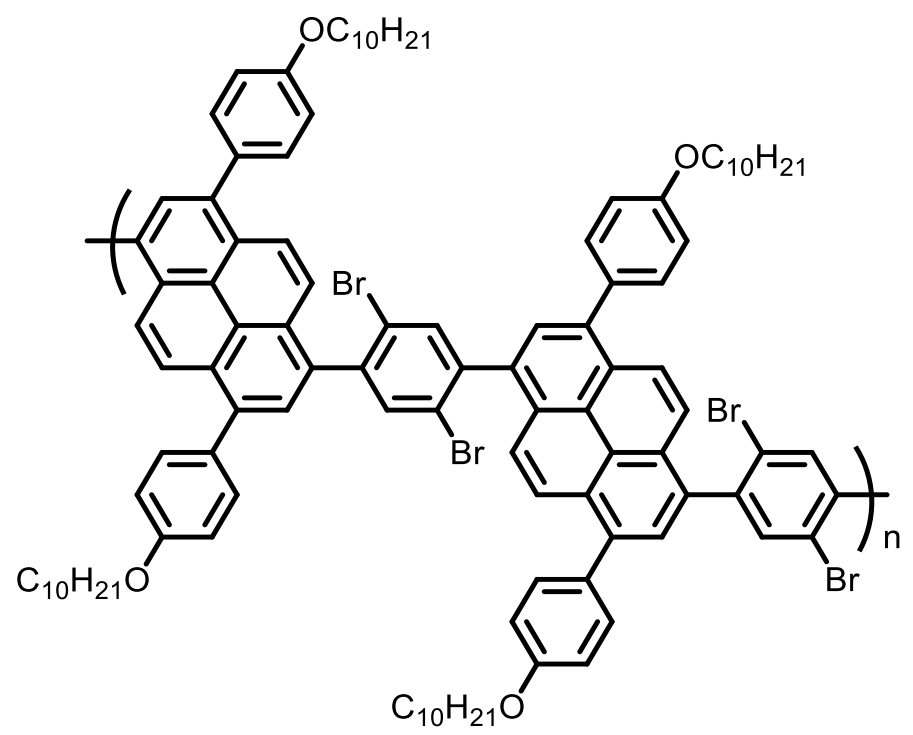

Poly[1,6-bis(4-(decyloxy)phenyl)-3-(2,5-dibromophenyl)pyrene] (17): In a glove box, 1,6dibromo-3,8-bis(4-(decyloxy)phenyl)pyrene (15) $(50 \mathrm{mg}, 0.061 \mathrm{mmol}), 2,2$ '-(2,5-dibromo-1,4phenylene)bis(4,4,5,5-tetramethyl-1,3,2-dioxaborolane) (5) (29.57 mg, $0.061 \mathrm{mmol}), \mathrm{Pd}\left(\mathrm{PPh}_{3}\right)_{4}$ $(7.05 \mathrm{mg}, 0.0061 \mathrm{mmol})$, potassium carbonate $(42.40 \mathrm{mg}, 0.31 \mathrm{mmol})$ and 1 drop of aliquot 336 were added in dry toluene $(1.8 \mathrm{~mL})$ and deionized water $(0.60 \mathrm{~mL})$ was added outside the glove box. The resulting mixture was refluxed at $110{ }^{\circ} \mathrm{C}$ for 3 days. Upon completion, the reaction was diluted with dichloromethane and water and extracted 3 times with DCM. After evaporation of the solvent, the reaction mixture was precipitated into $100 \mathrm{~mL}$ methanol, filtered, washed with copious amount of acetone and dried over high vacuum. Recycling preparative GPC was used to further purify the polymer to $19 \mathrm{mg}(35 \%)$ as yellow solid. ${ }^{1} \mathrm{H}$ NMR $\left(400 \mathrm{MHz}, \mathrm{CDCl}_{3}\right) \delta 8.31-7.48(\mathrm{~m}$, 
$J=124.5 \mathrm{~Hz}, 24 \mathrm{H}), 7.09-6.96(\mathrm{~m}, 8 \mathrm{H}), 4.09-3.80(\mathrm{~m}, 8 \mathrm{H}), 1.88-1.64(\mathrm{~m}, 8 \mathrm{H}), 1.55-1.39(\mathrm{~m}$, $J=28.3 \mathrm{~Hz}, 8 \mathrm{H}), 1.31-1.12(\mathrm{~m}, 48 \mathrm{H}), 0.86-0.74(\mathrm{~m}, 12 \mathrm{H})$.

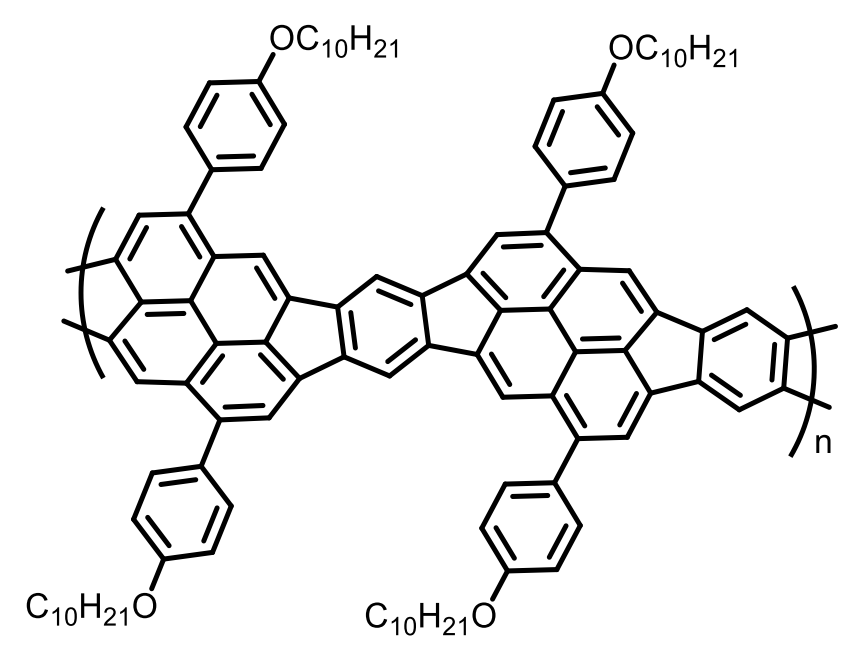

5,12-bis(4-(decyloxy)phenyl)indeno[1,2,3-cd]pyrene (2b): In the glove box, Poly[1,6-bis(4(decyloxy)phenyl)-3-(2,5-dibromophenyl)pyrene] (20) (15 mg, $0.0084 \mathrm{mmol}), \mathrm{Pd}\left(\mathrm{PCy}_{3}\right)_{2} \mathrm{Cl}_{2}(6$ $\mathrm{mg}, 0.0075 \mathrm{mmol})$, DBU $(0.10 \mathrm{~mL})$, and DMA $(2 \mathrm{~mL})$ were added to a $10 \mathrm{~mL}$ seal tube. The seal tube was removed from the glove box and placed into a preheated oil bath where it was heated at $140{ }^{\circ} \mathrm{C}$ for overnight. After cooling, the reaction mixture was poured into $100 \mathrm{~mL}$ methanol, filtered, washed with copious amount of acetone, dichloromethane and dried over high vacuum to give $9 \mathrm{mg}(75 \%)$ as purple solid. We are unable to get molecular weight due to the almost insolubility of polymer in tetrahydrofuran. But we have found ${ }^{1} \mathrm{H}$ NMR owing to very tiny solubility of polymer in chloroform. ${ }^{1} \mathrm{H} \mathrm{NMR}\left(400 \mathrm{MHz}, \mathrm{CDCl}_{3}\right) \delta 8.51-7.49(\mathrm{~m}, J=71.2 \mathrm{~Hz}$, $20 \mathrm{H}), 7.15-6.84(\mathrm{~m}, 8 \mathrm{H}), 4.46-3.81(\mathrm{~m}, 8 \mathrm{H}), 1.38-0.65(\mathrm{~m}, J=91.9,64.1,48.2 \mathrm{~Hz}, 76 \mathrm{H})$. 
Compound 3<smiles>[Te]</smiles>
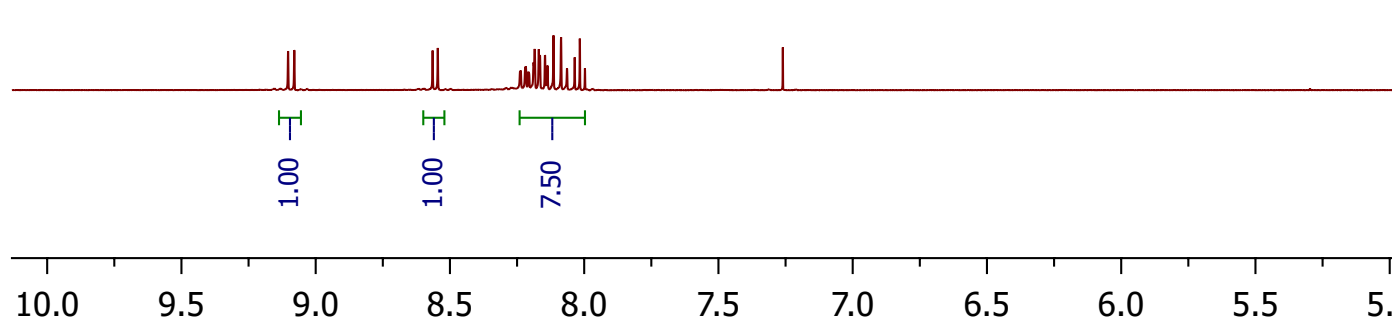

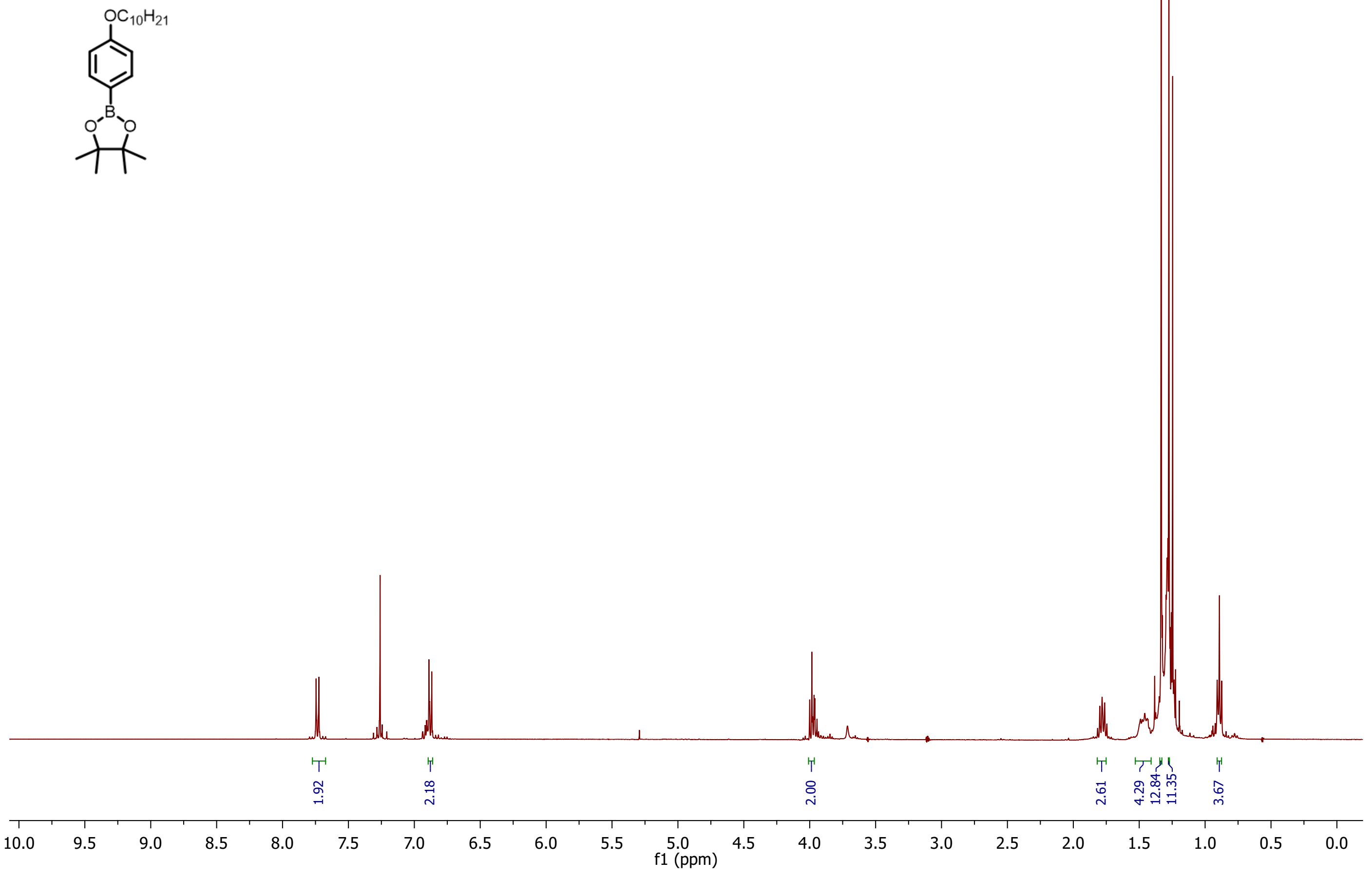

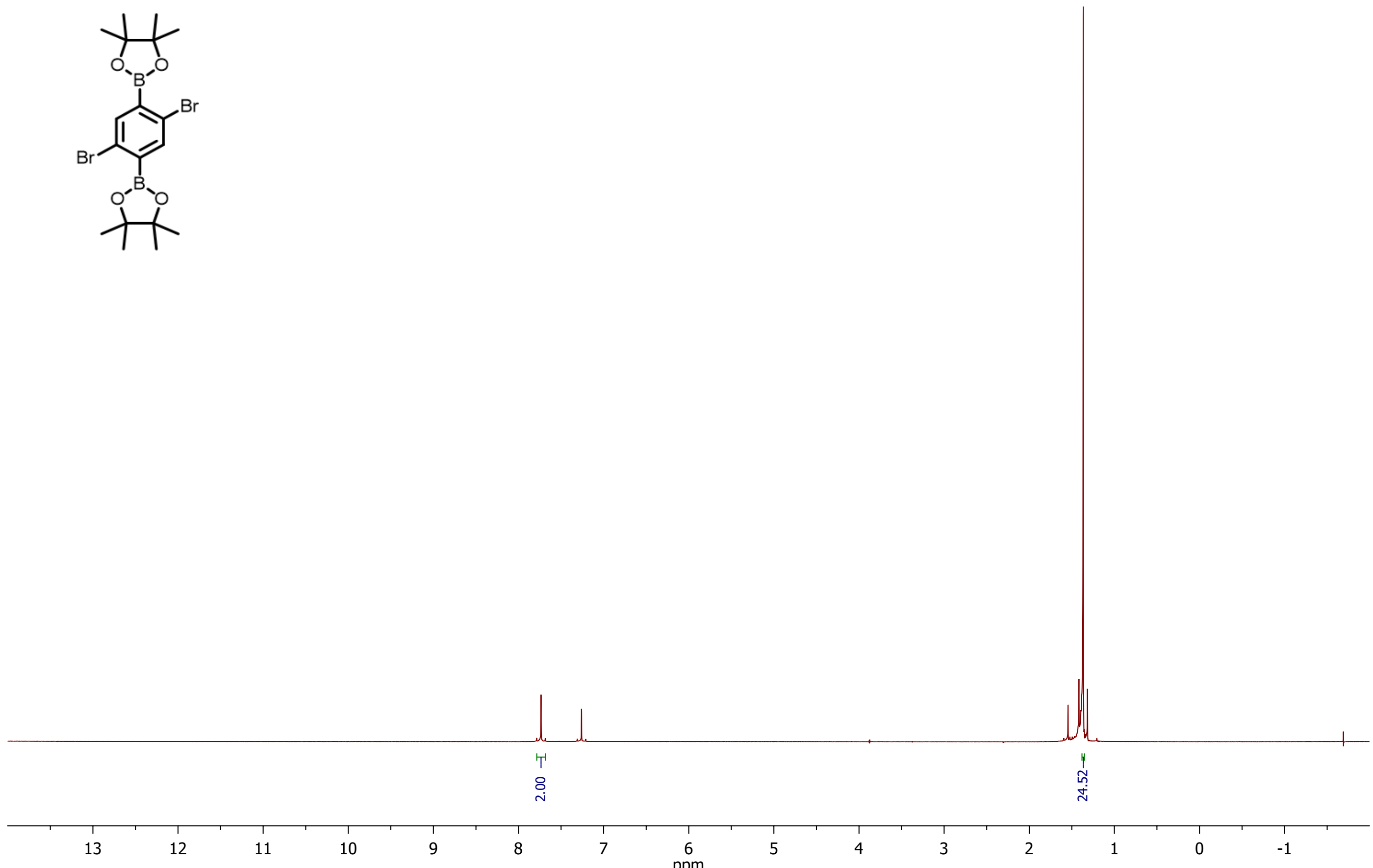
<smiles>[3H]</smiles>

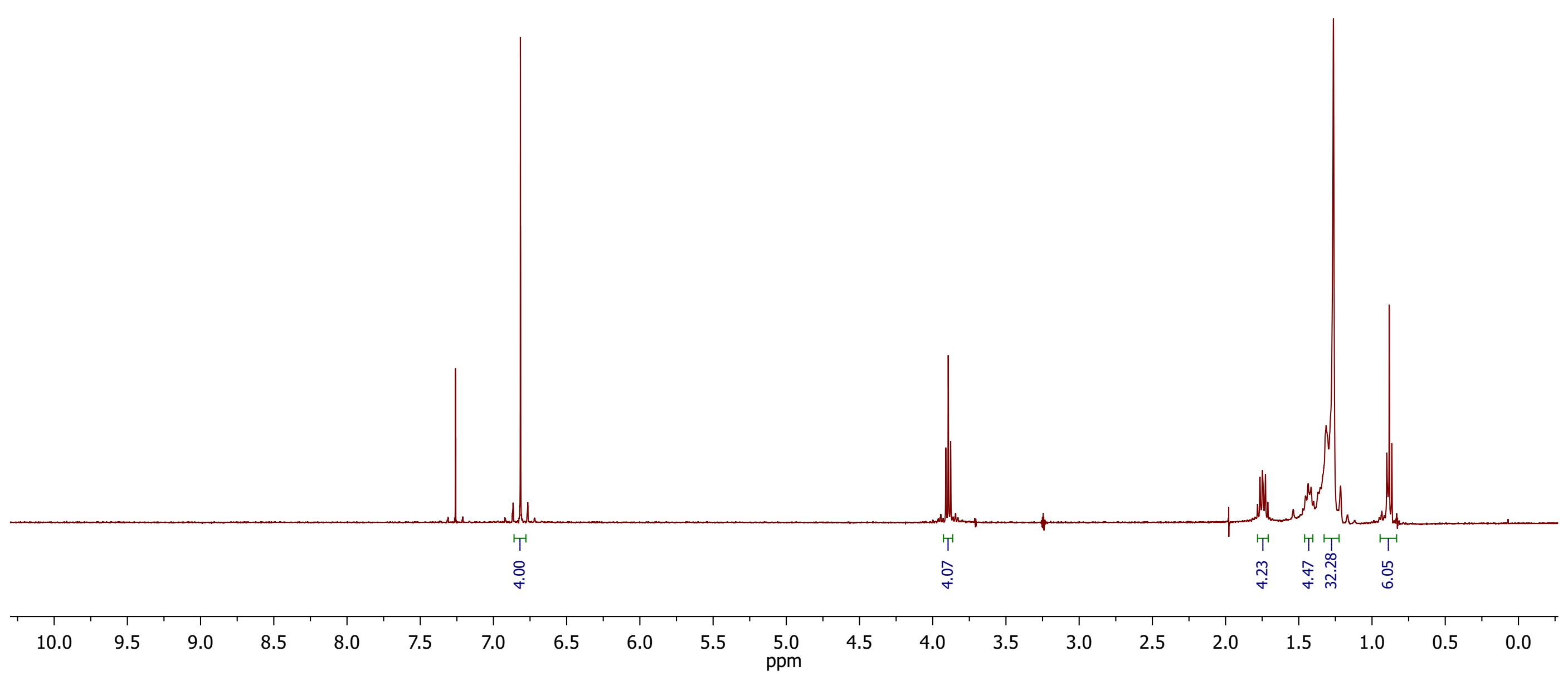



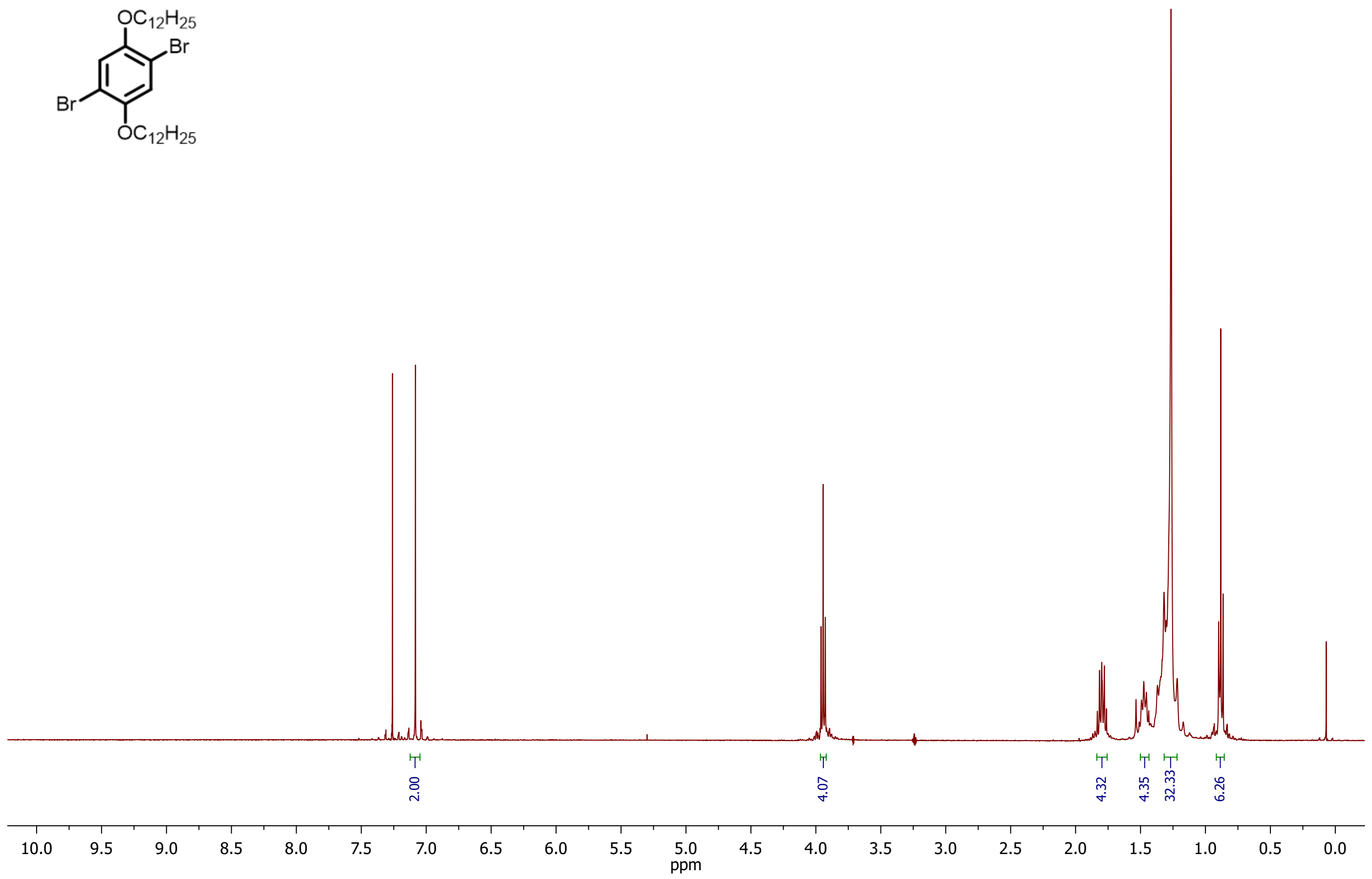
Compound 5<smiles>CCCCCCOc1cc(-c2ccc3ccc4cccc5ccc2c3c45)c(OCCCC)cc1-c1ccc2ccc3cccc4ccc1c2c34</smiles>

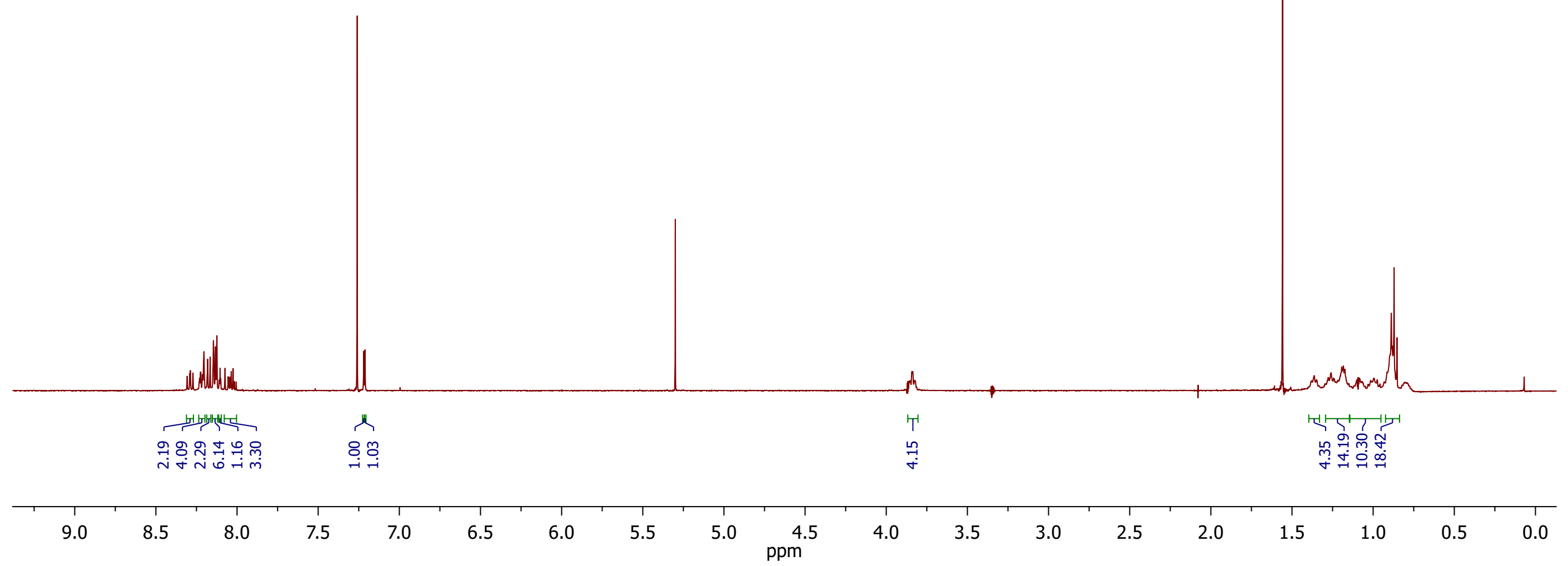




等



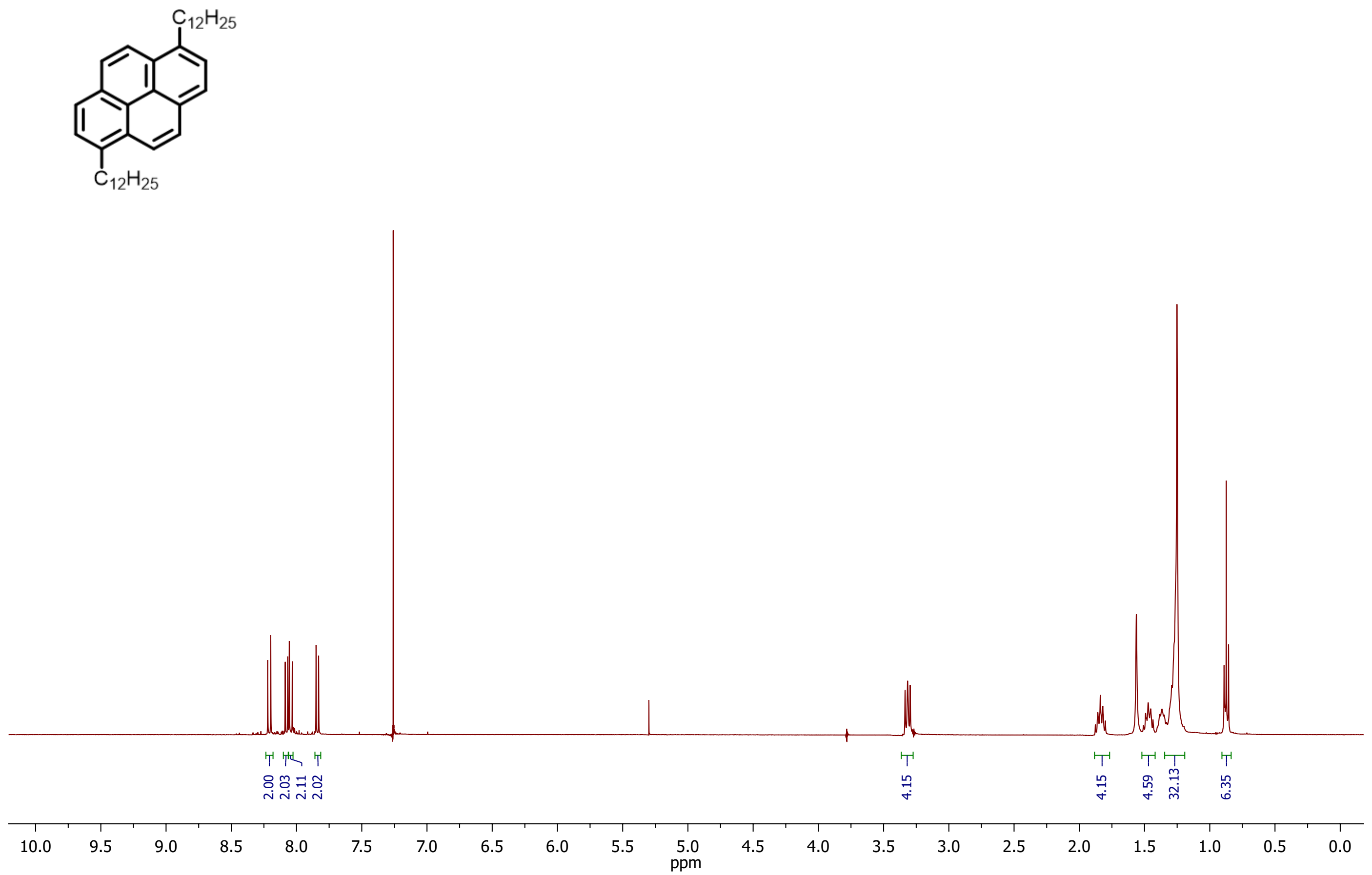
<smiles>[Se]=[Se]</smiles>

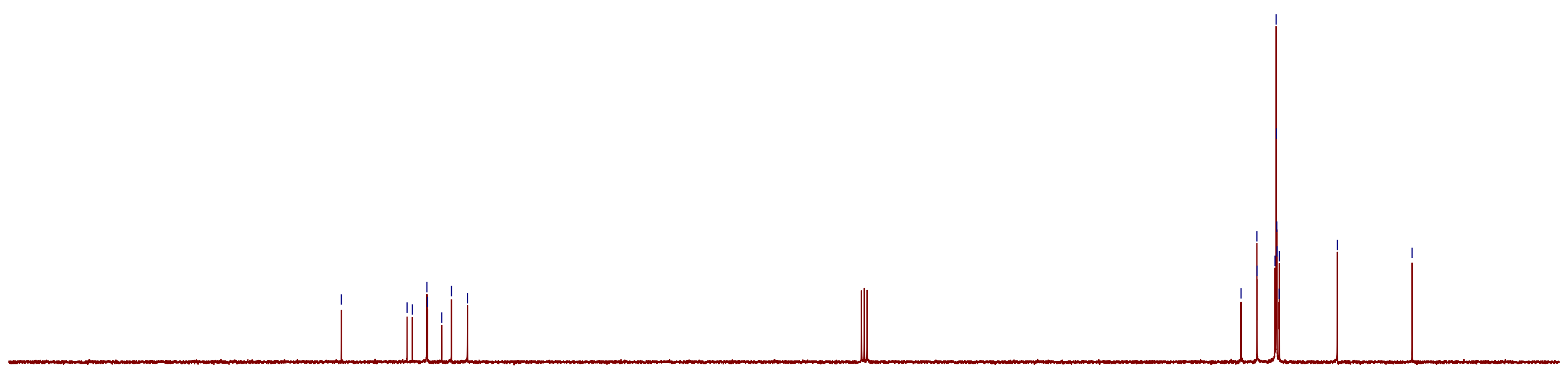

\begin{tabular}{|c|c|c|c|c|c|c|c|c|c|c|c|c|c|c|c|c|c|}
\hline $\begin{array}{c}1 \\
170\end{array}$ & 1 & $\begin{array}{c}11 \\
150\end{array}$ & 14 & $\begin{array}{c}11 \\
130\end{array}$ & 12 & 11 & $\begin{array}{c}11 \\
100\end{array}$ & I & 80 & $\begin{array}{l}1 \\
70\end{array}$ & 1 & $\begin{array}{l}1 \\
50\end{array}$ & 1 & $\begin{array}{l}1 \\
30\end{array}$ & 13 & ${ }_{10}^{1}$ & 1 \\
\hline 170 & 160 & 150 & 140 & 130 & 120 & 110 & 100 & 90 & 80 & 70 & 60 & 50 & 40 & 30 & 20 & 10 & 0 \\
\hline
\end{tabular}



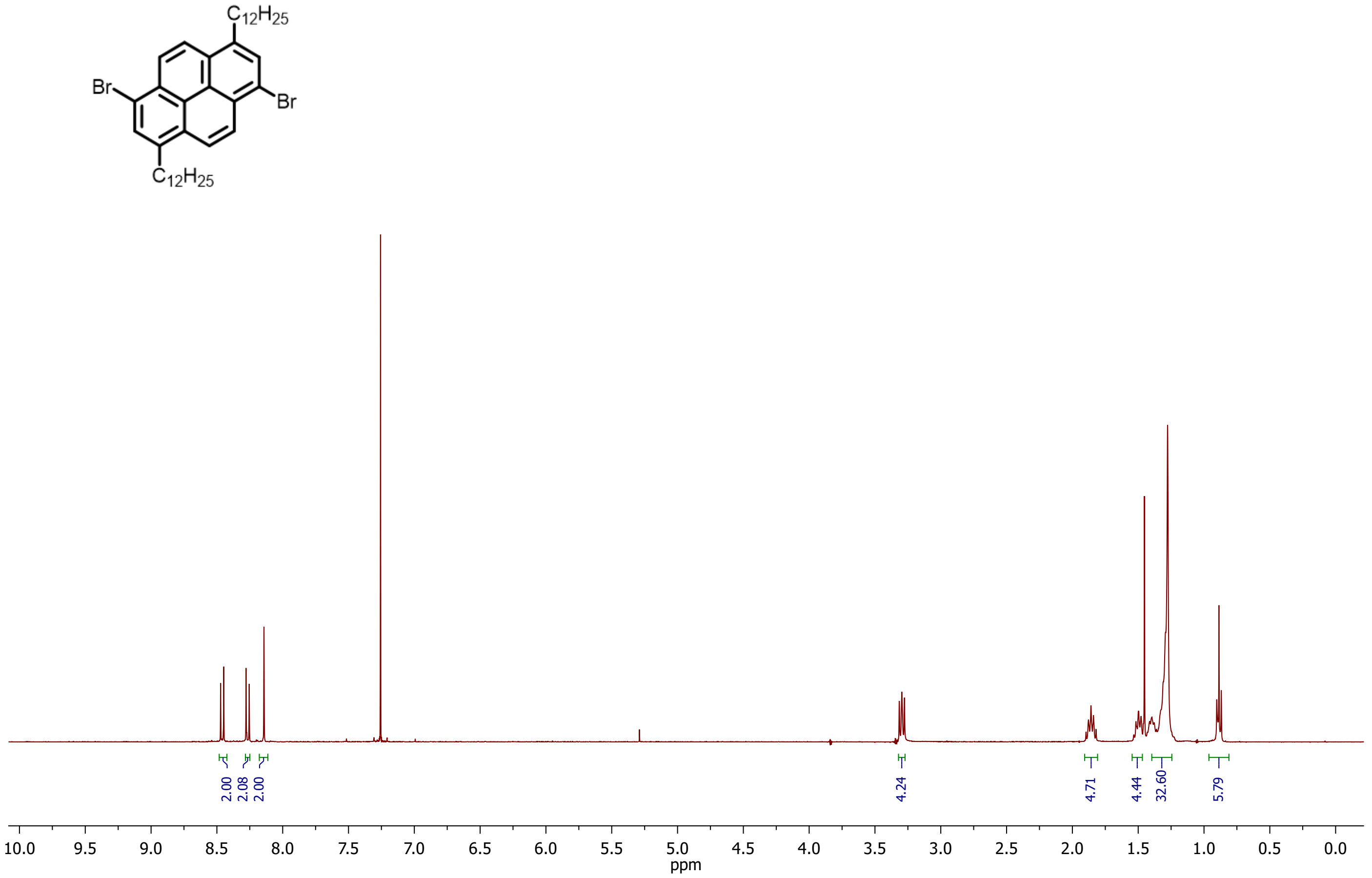


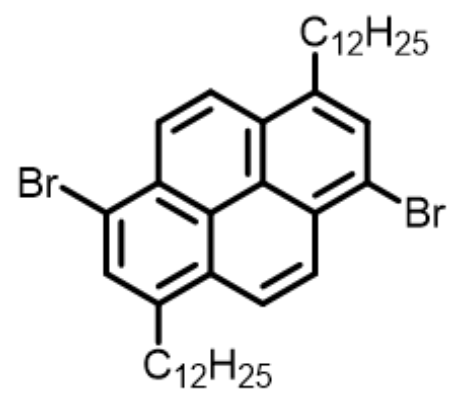
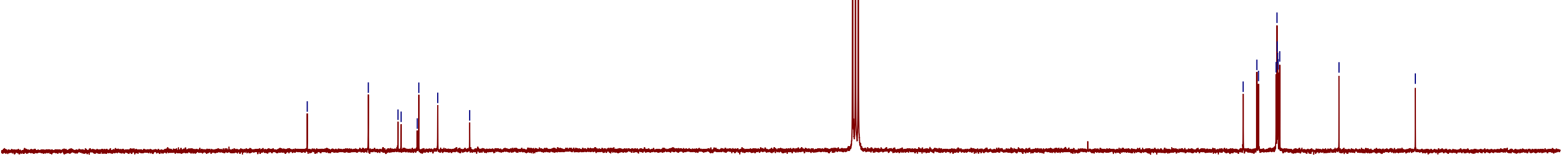

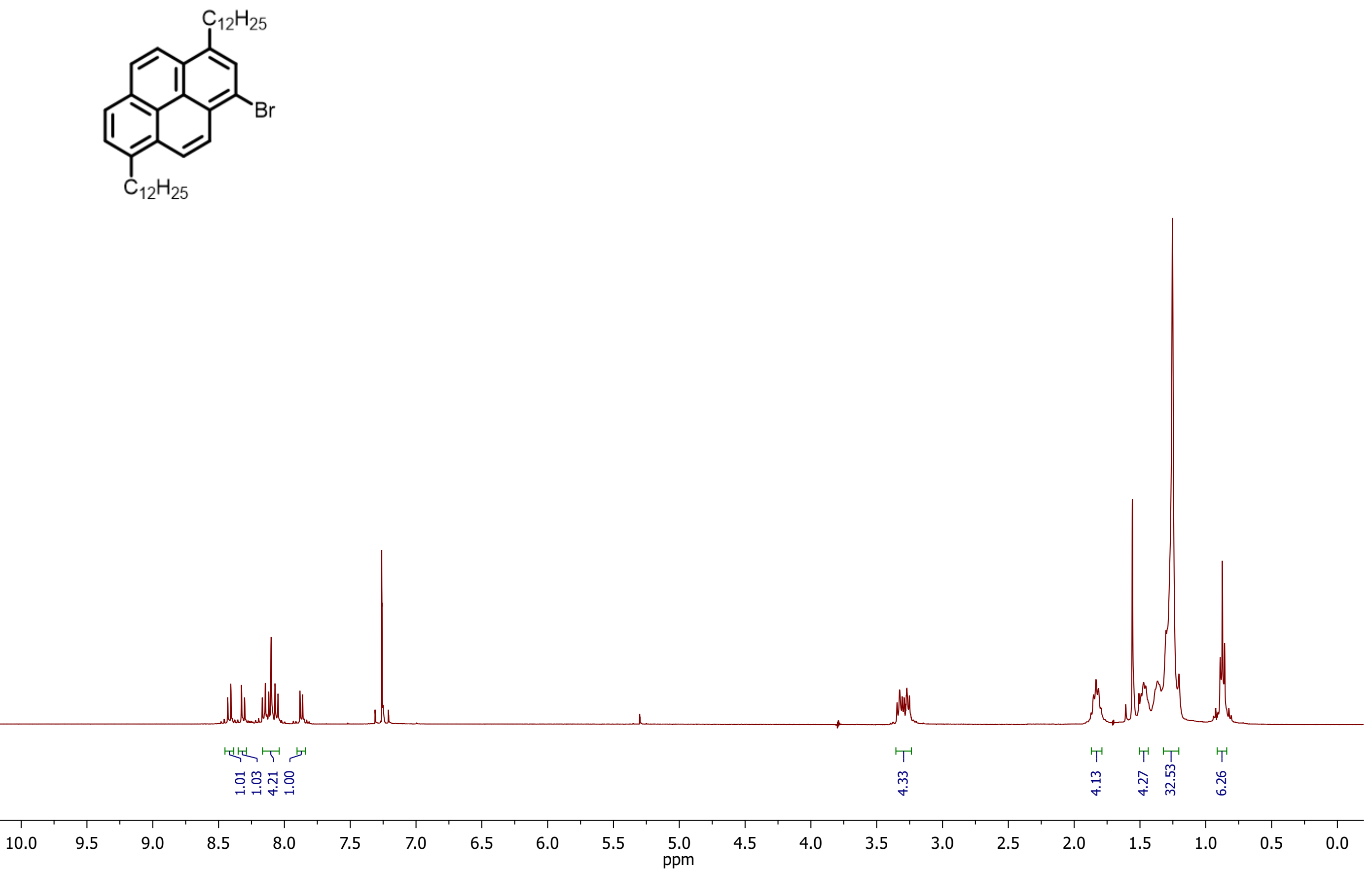

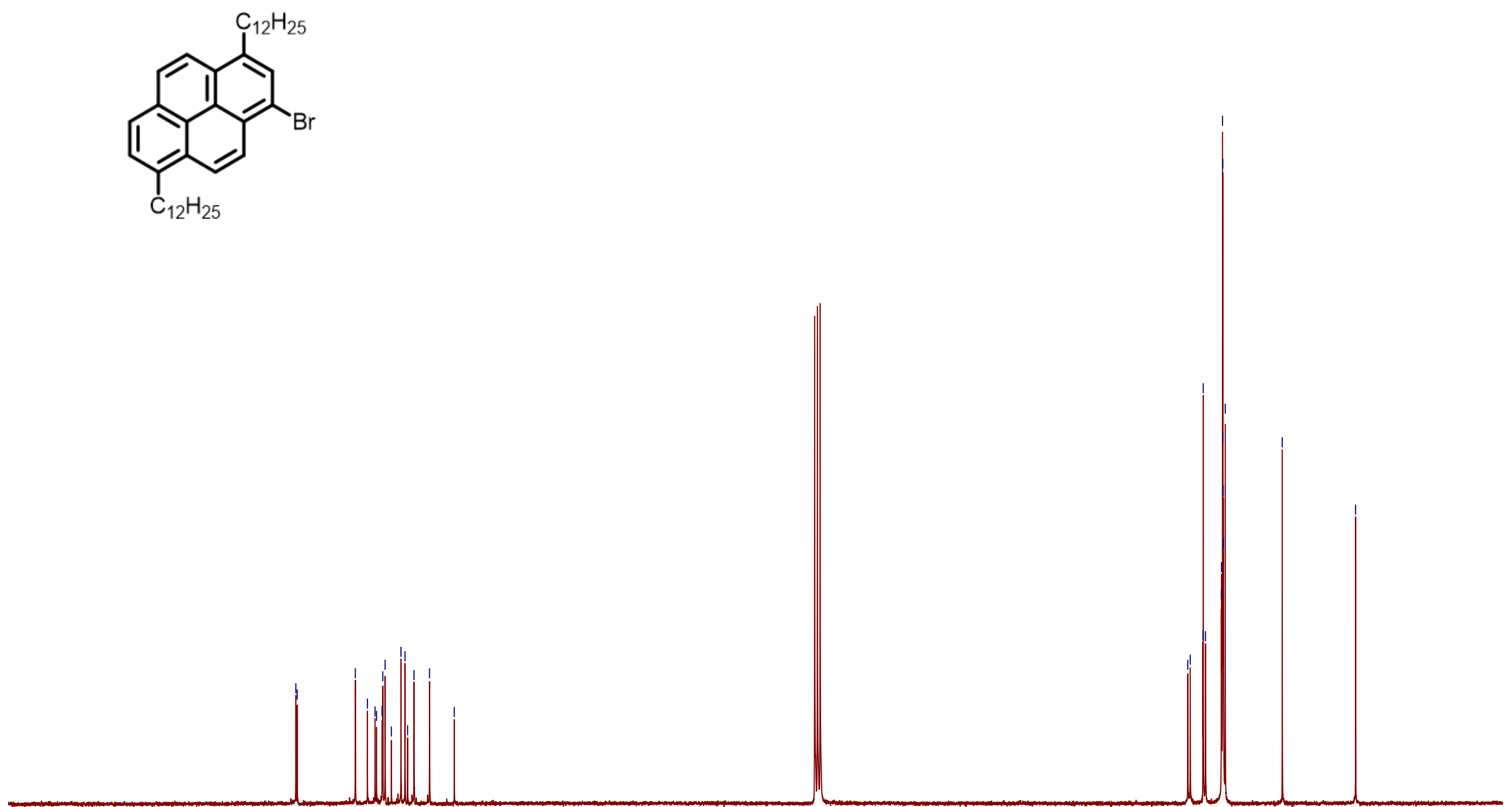

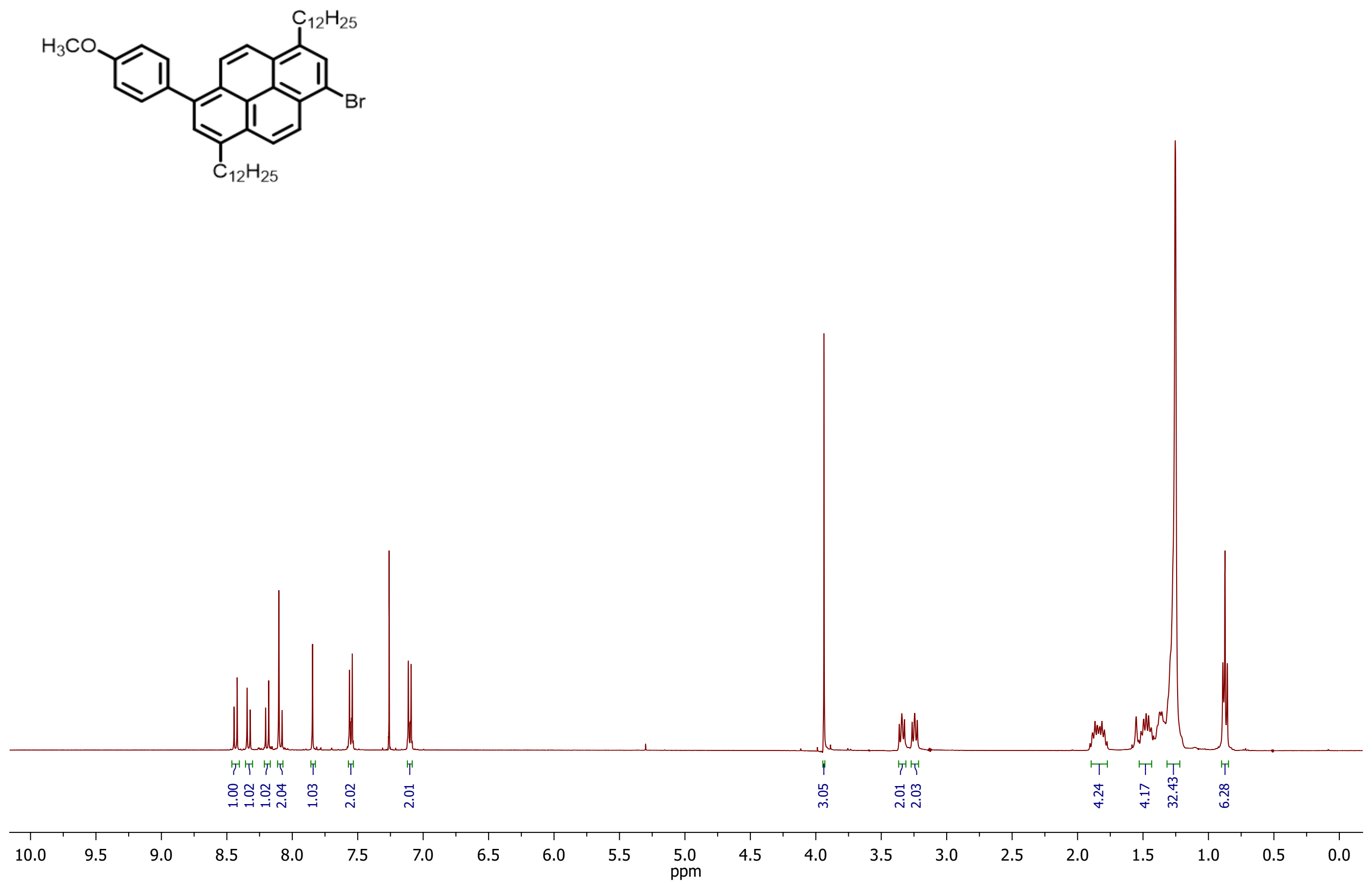


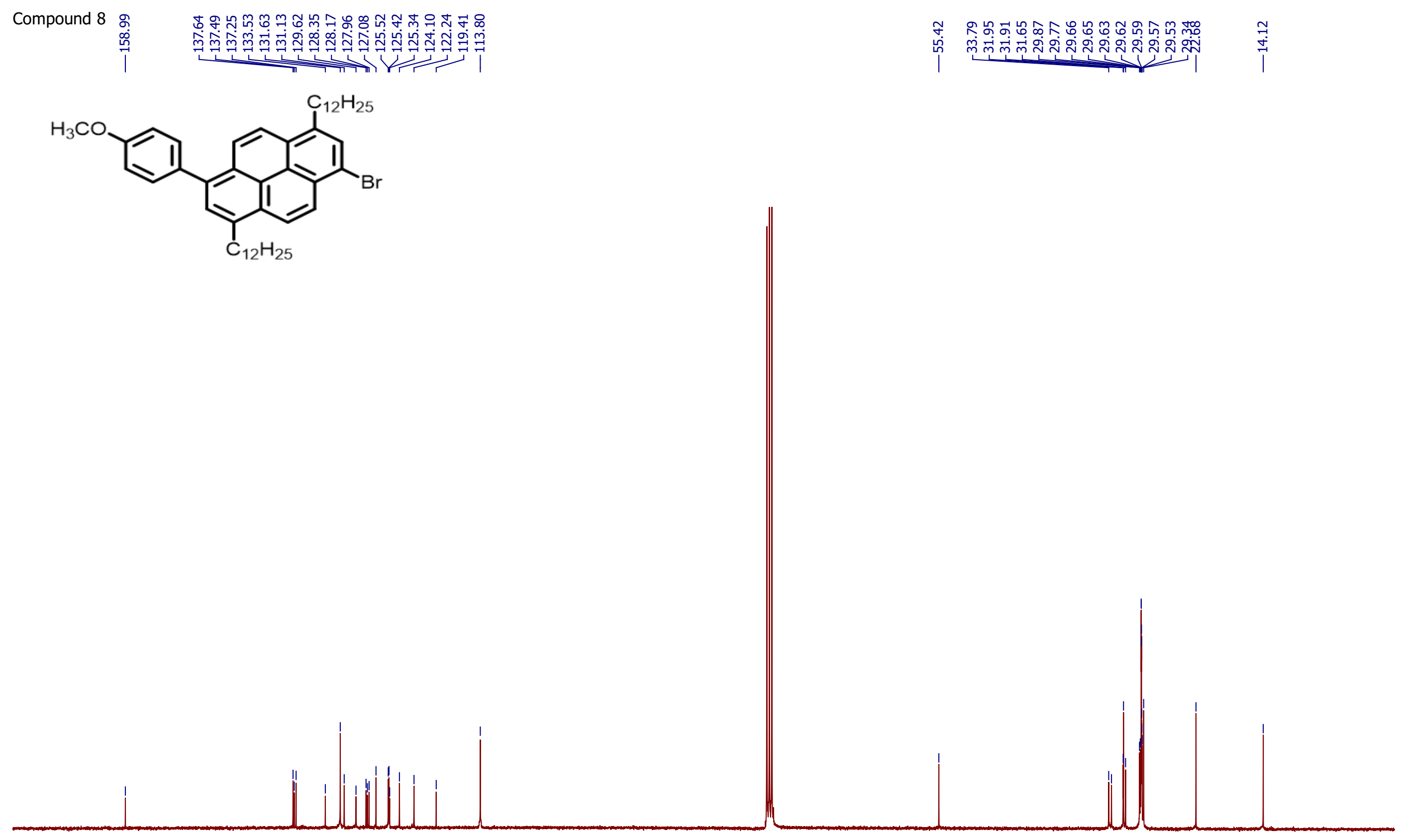

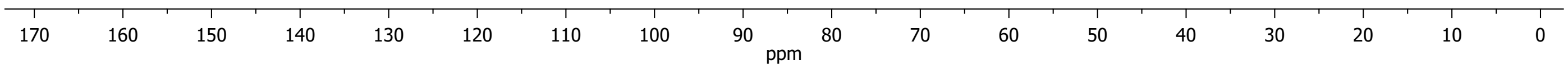



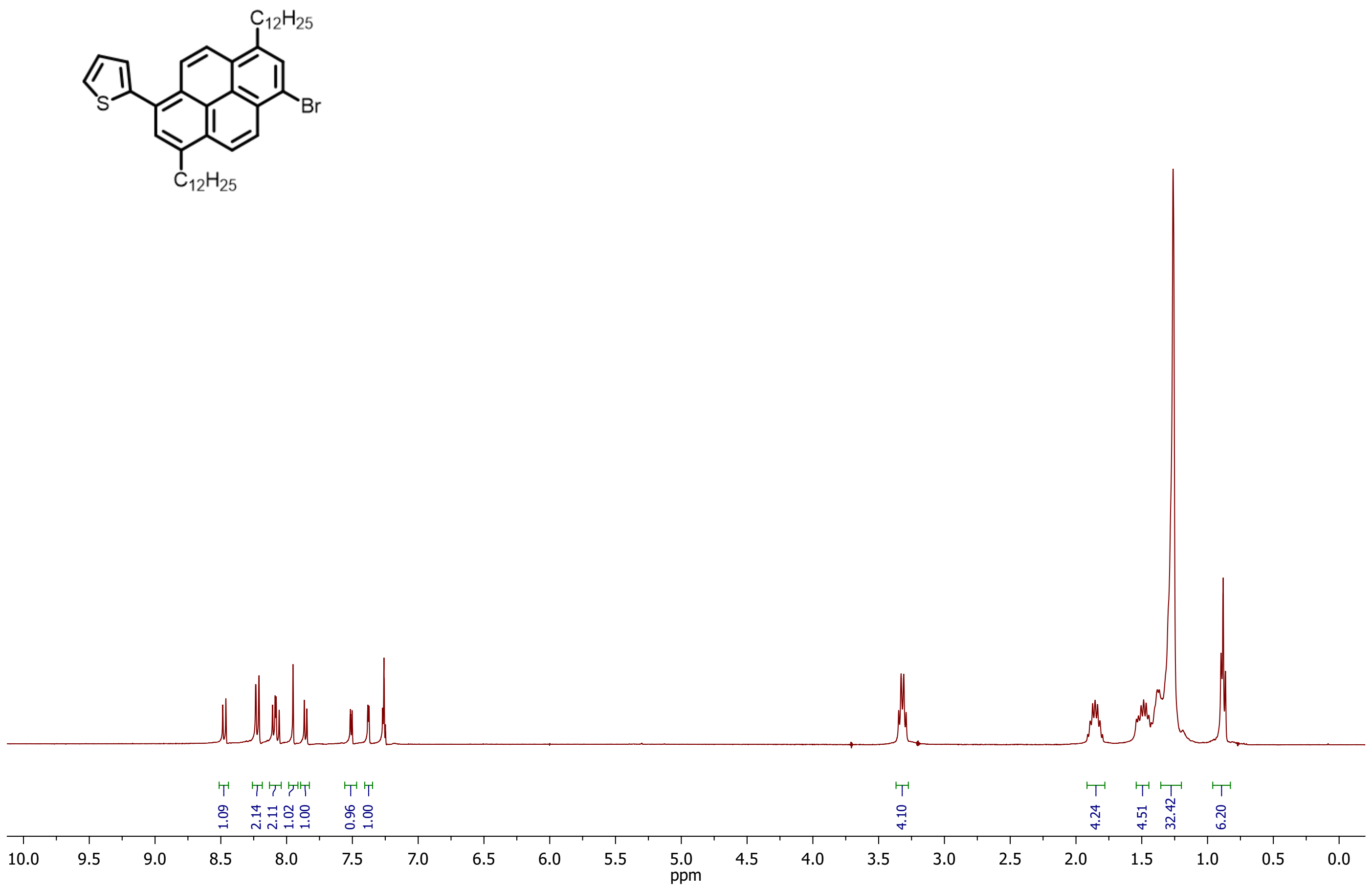


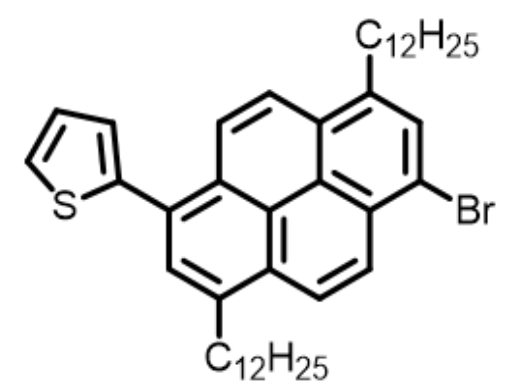

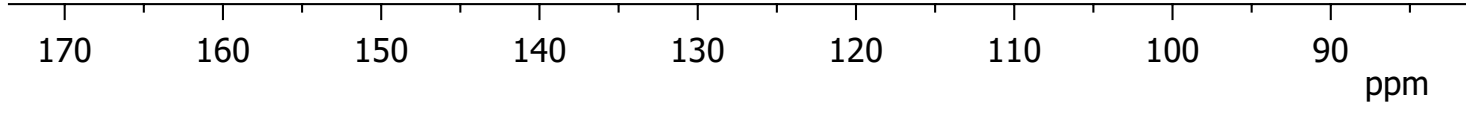



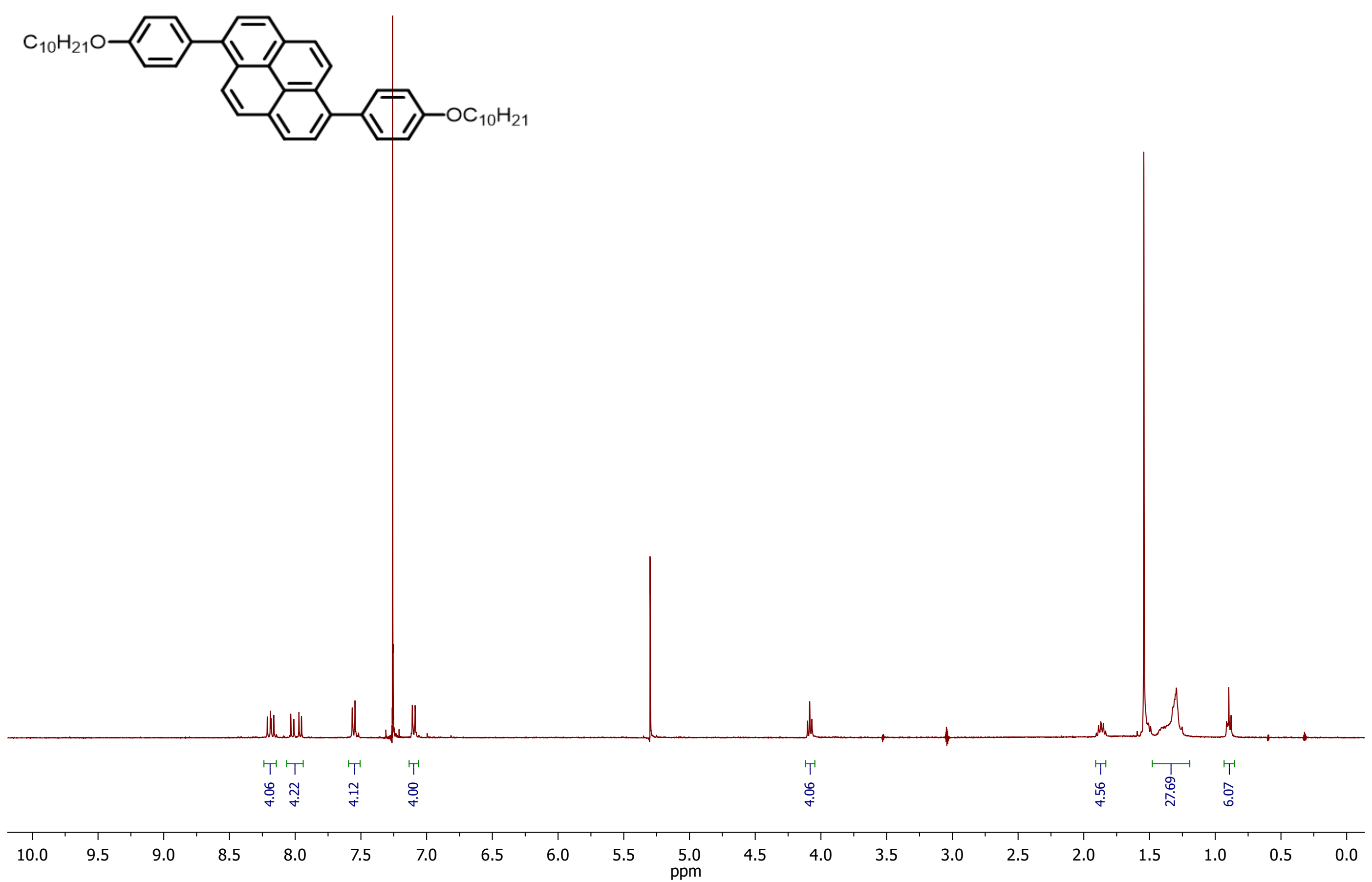


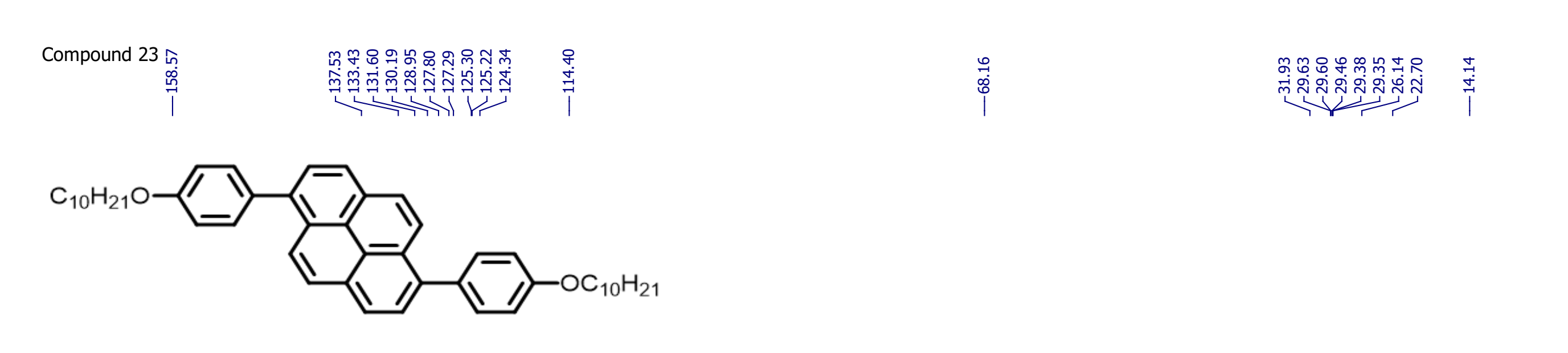



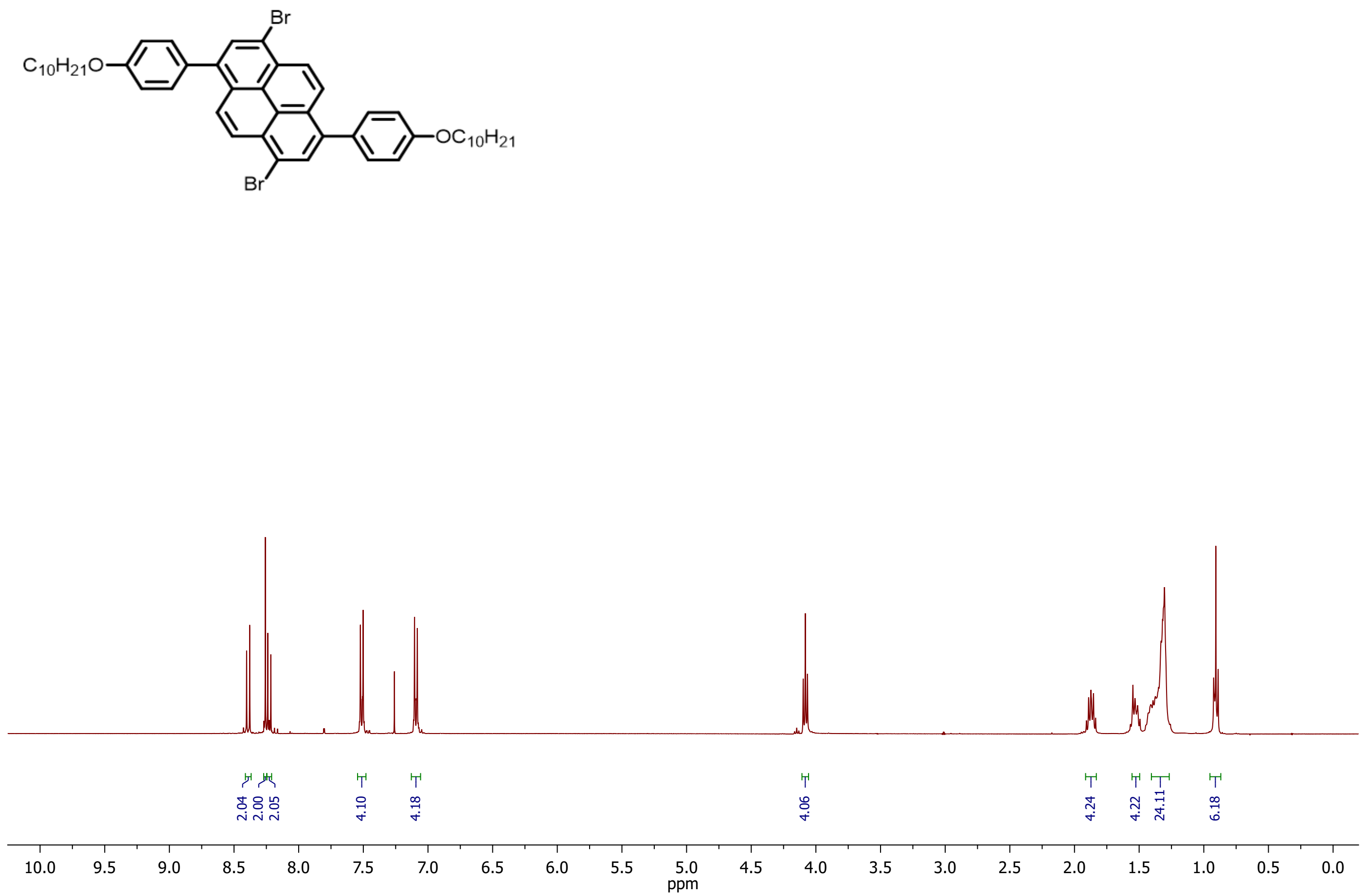

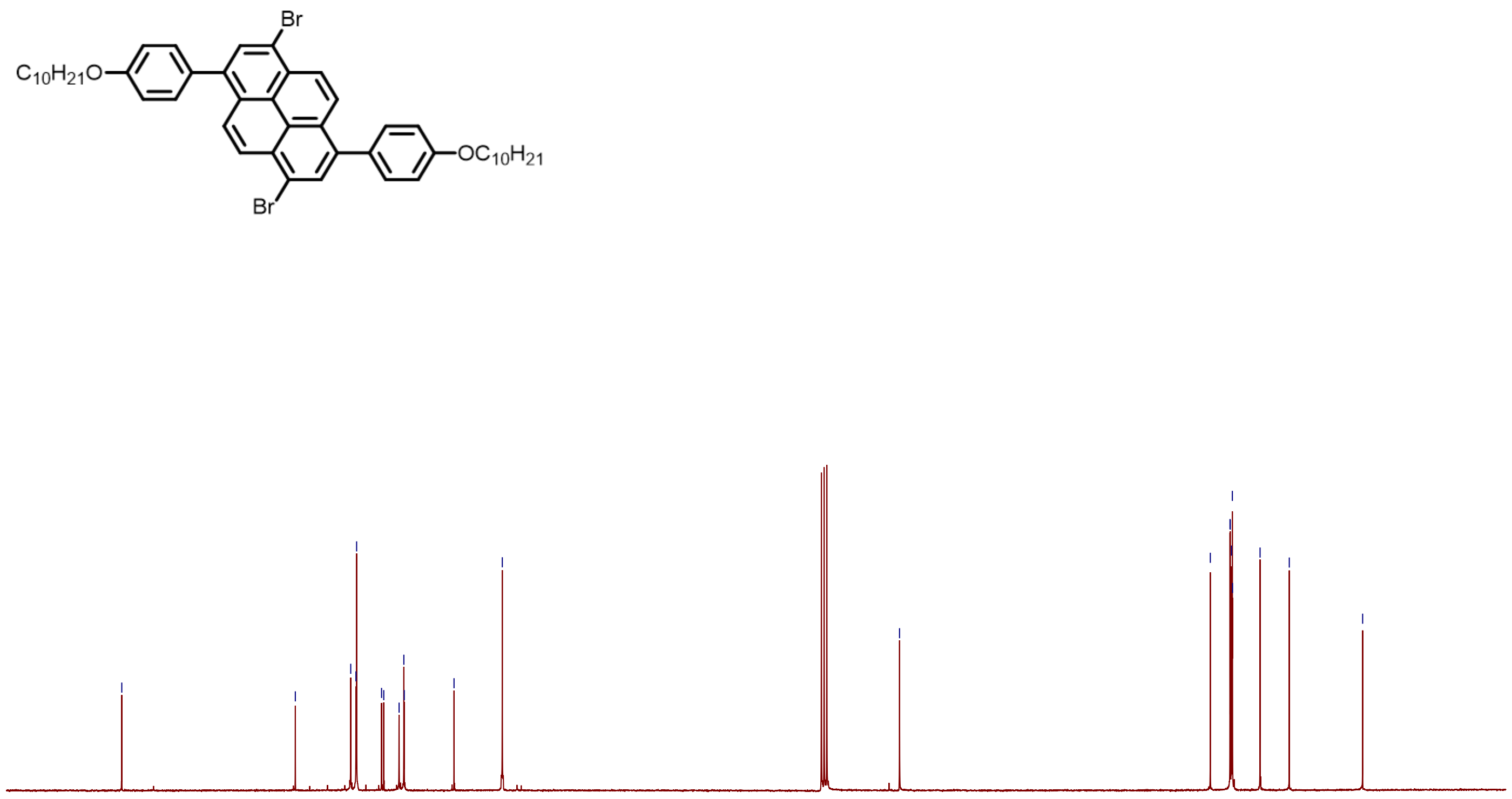


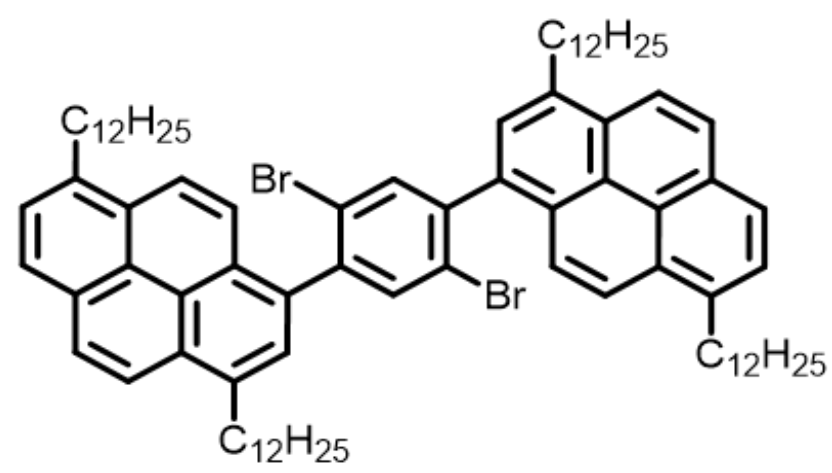

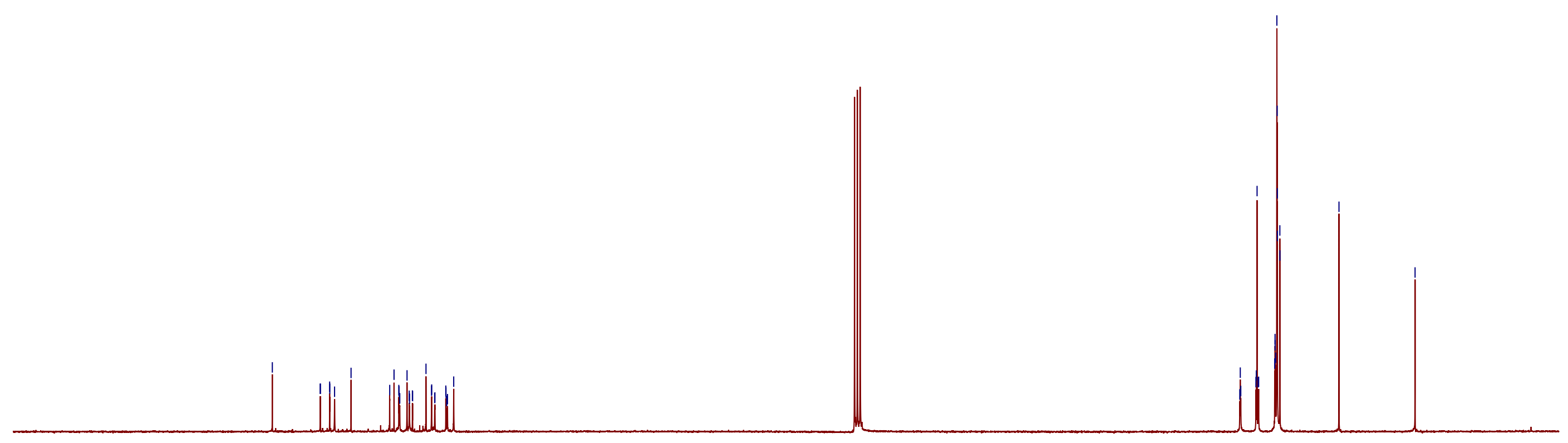



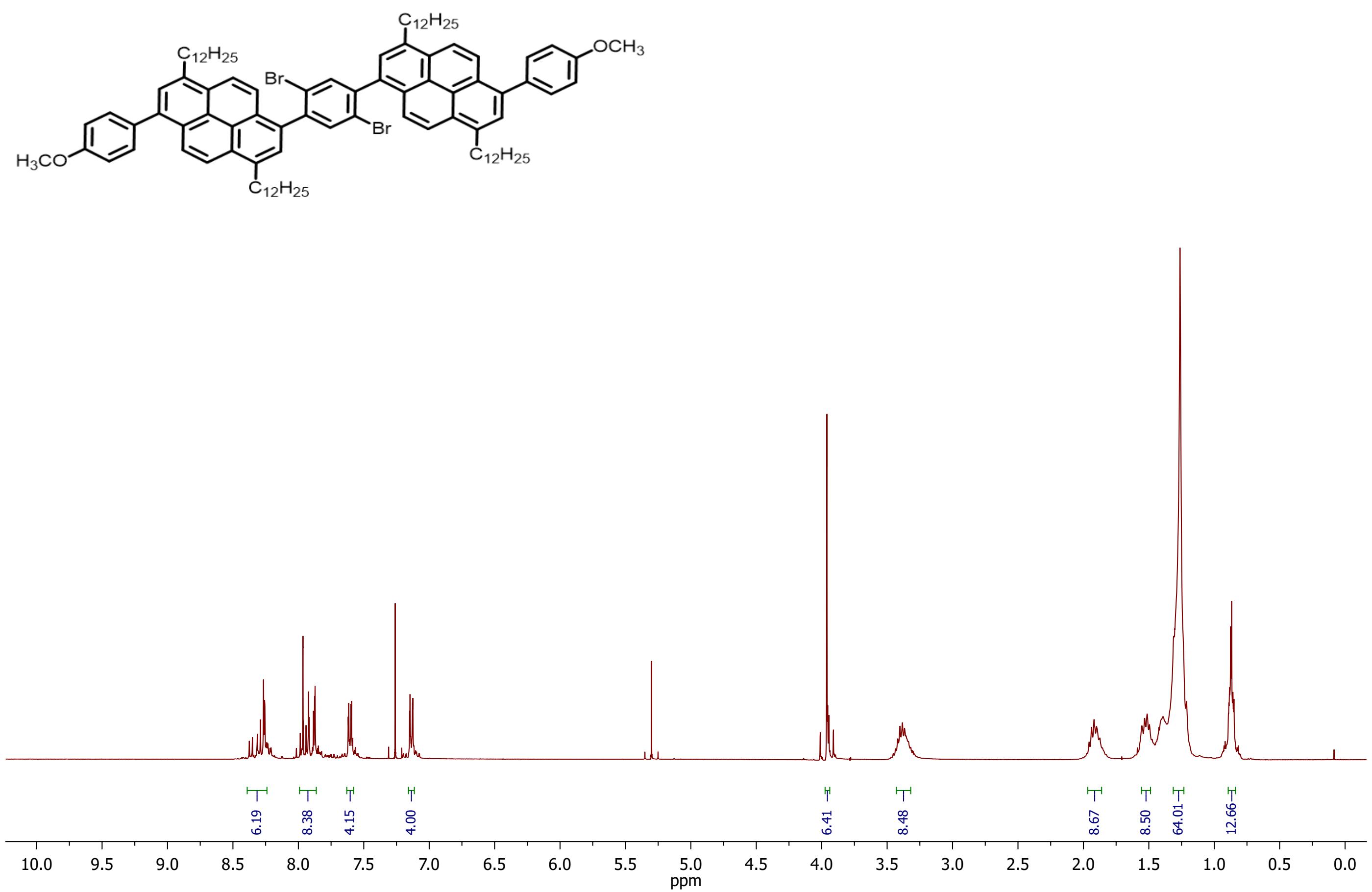

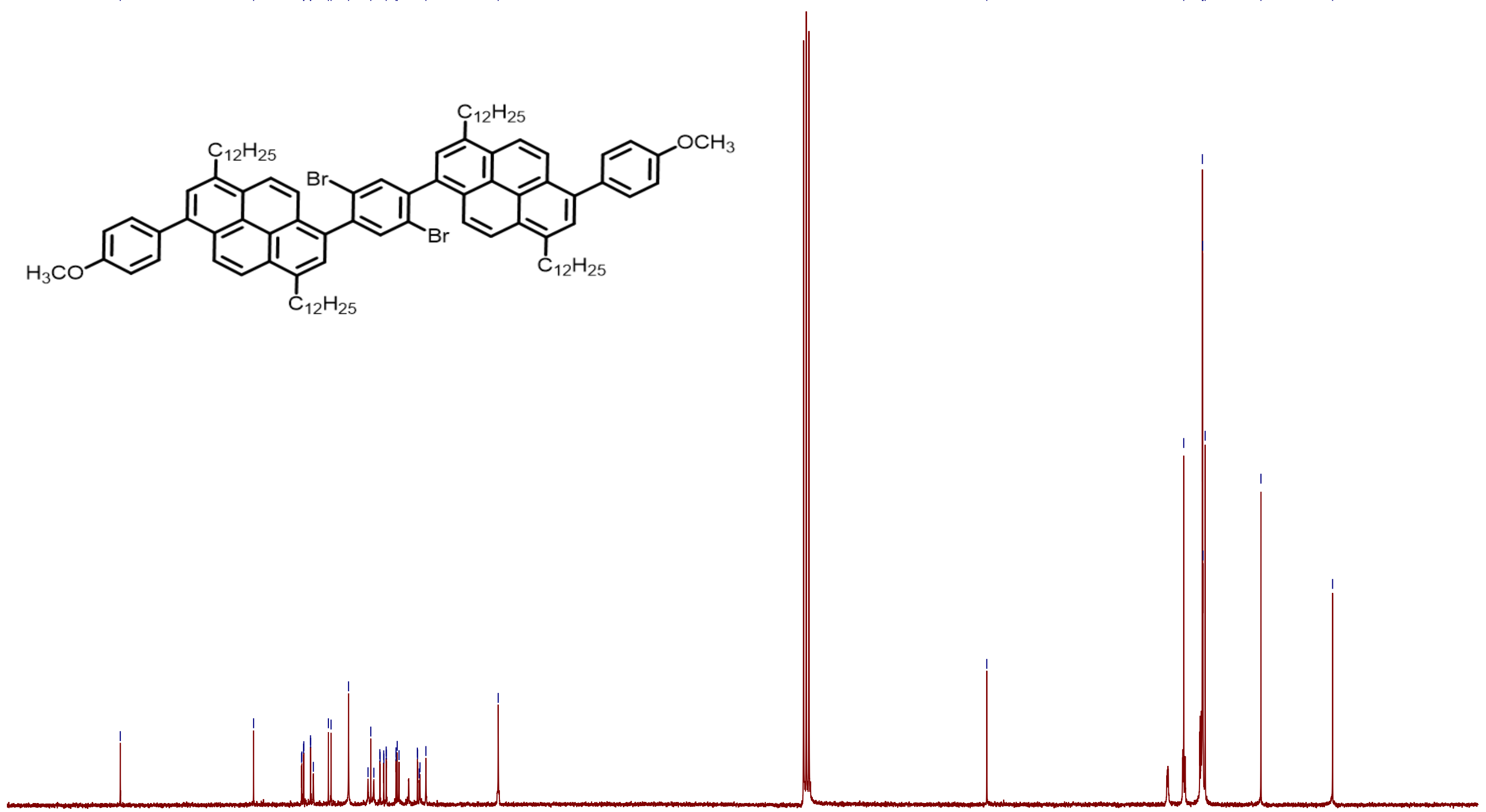

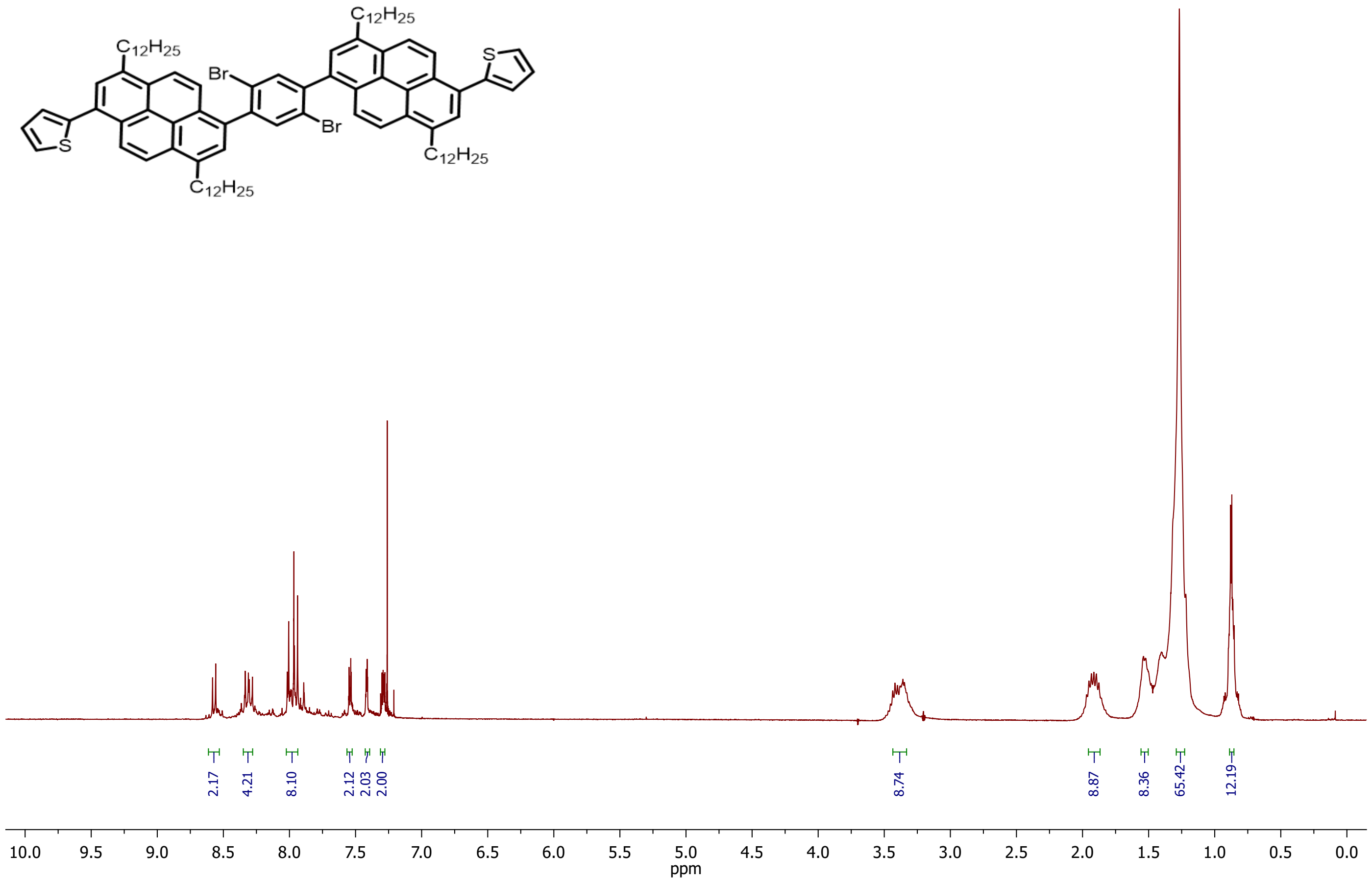

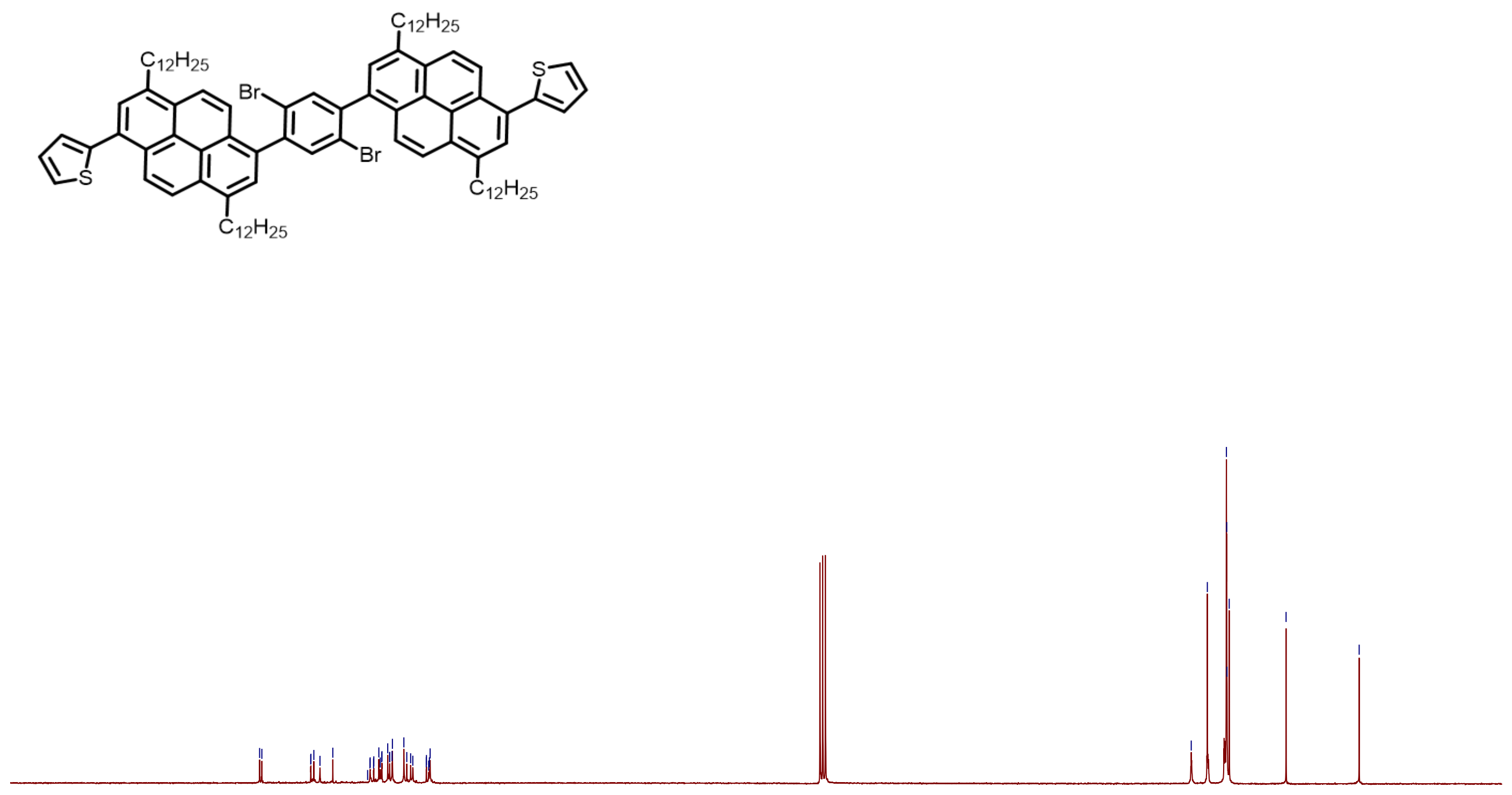

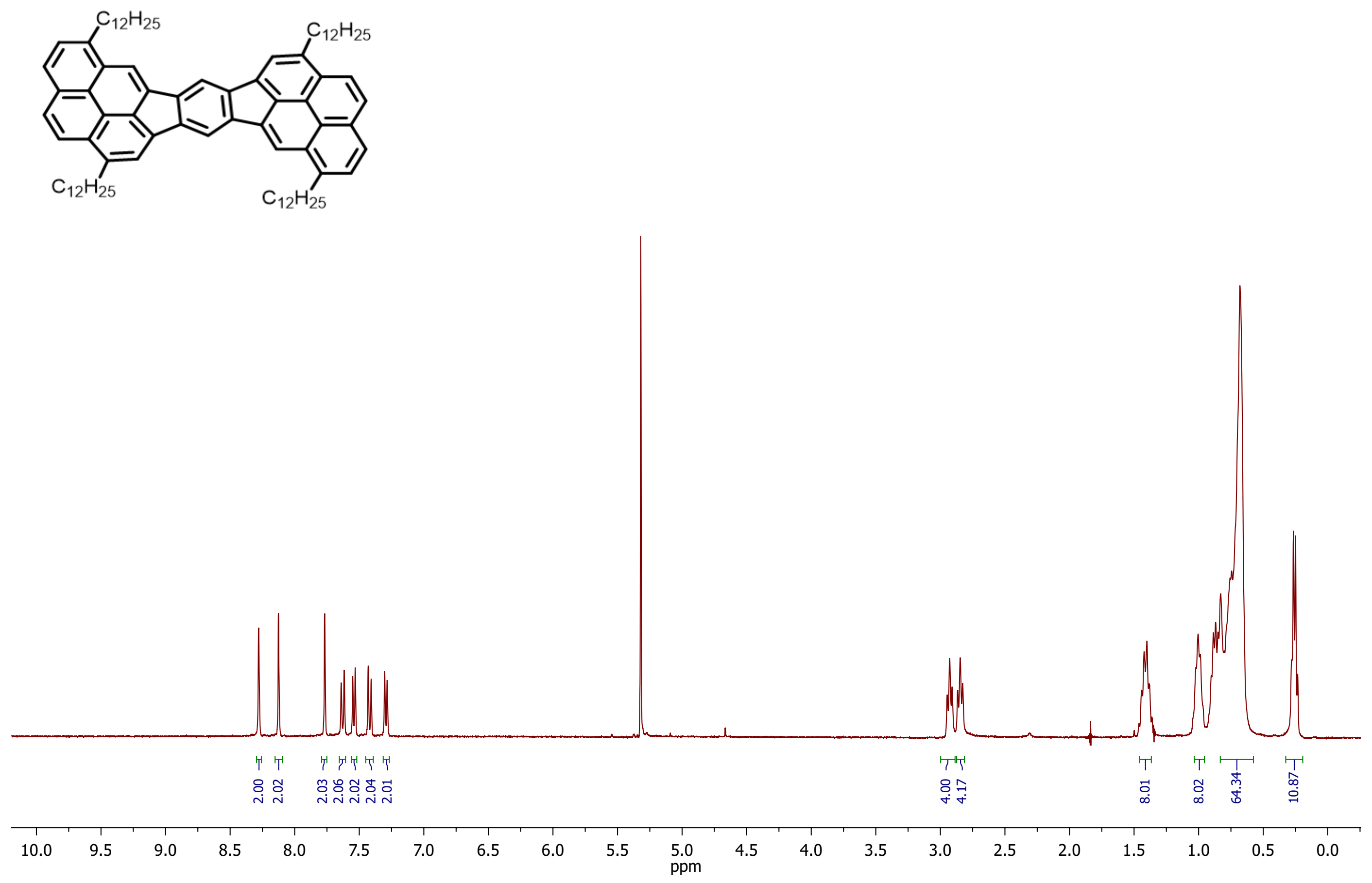

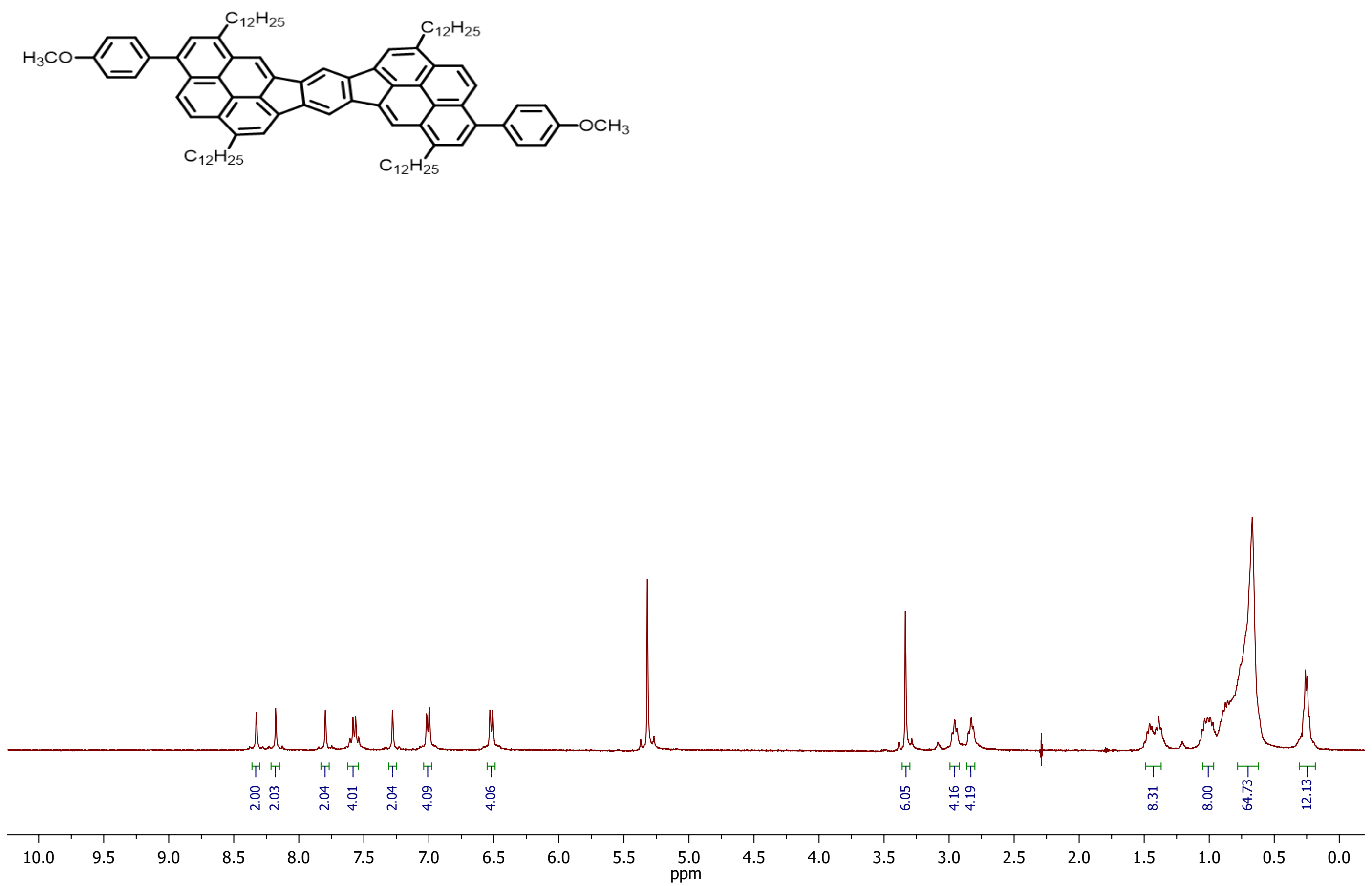

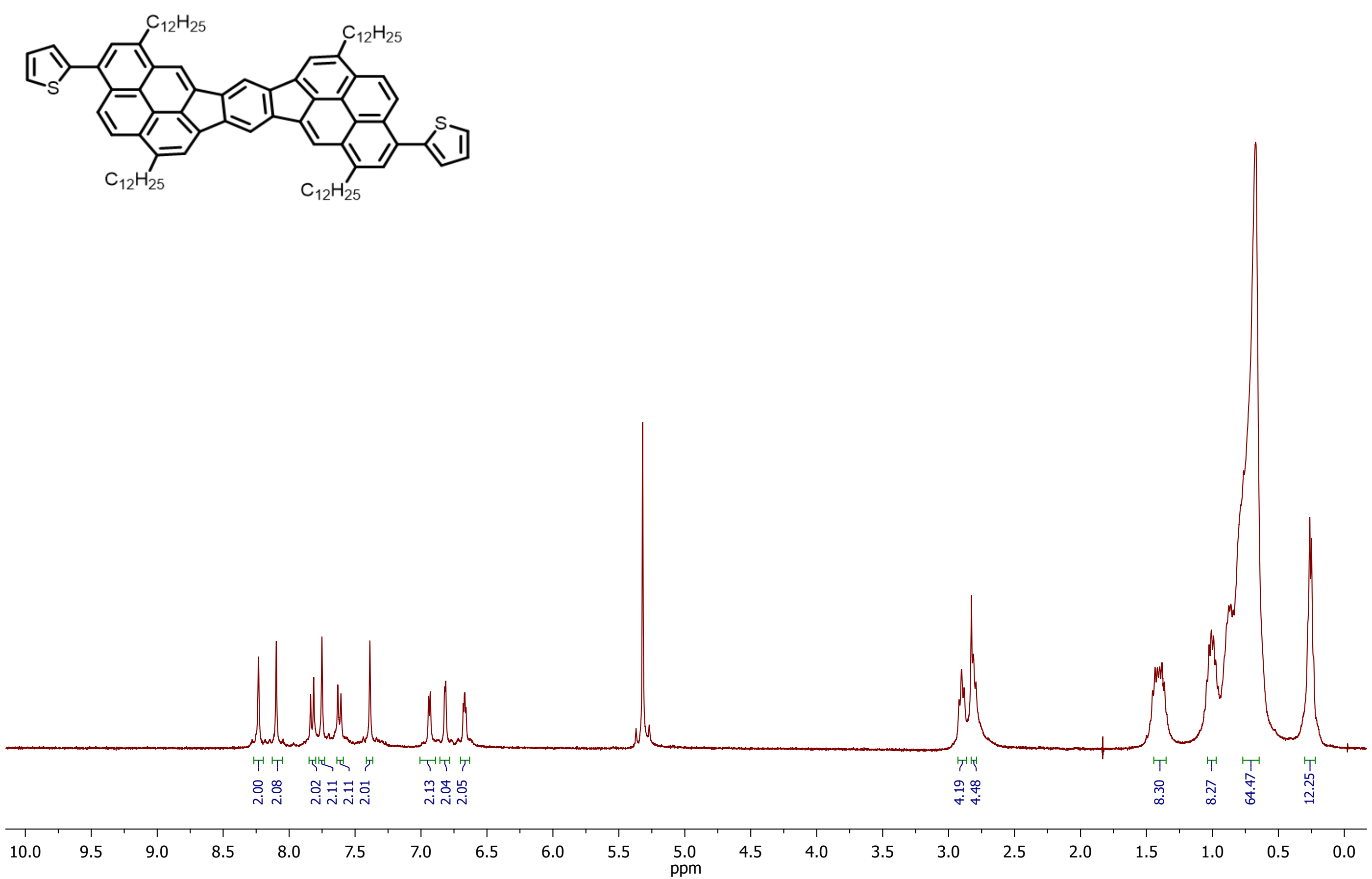


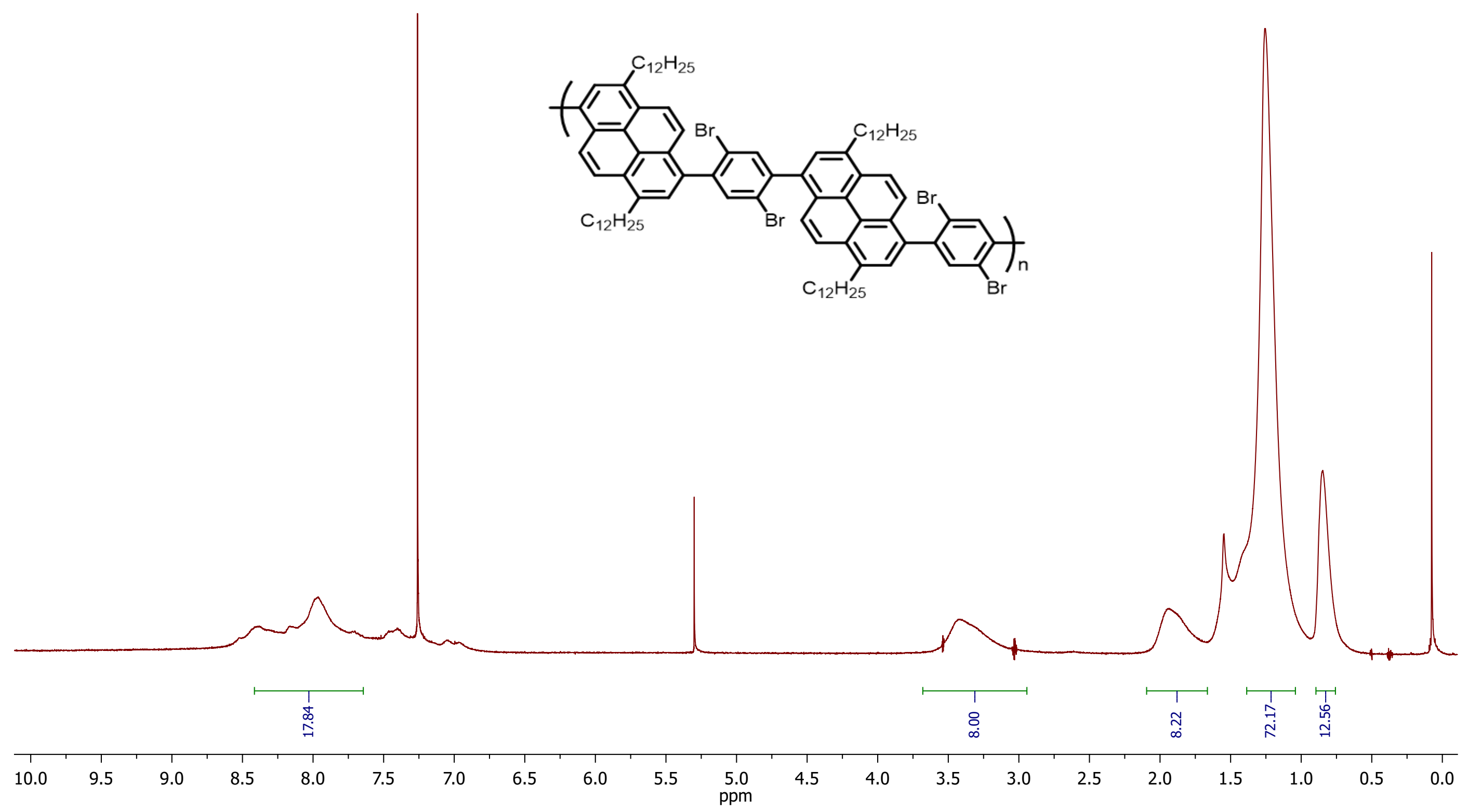



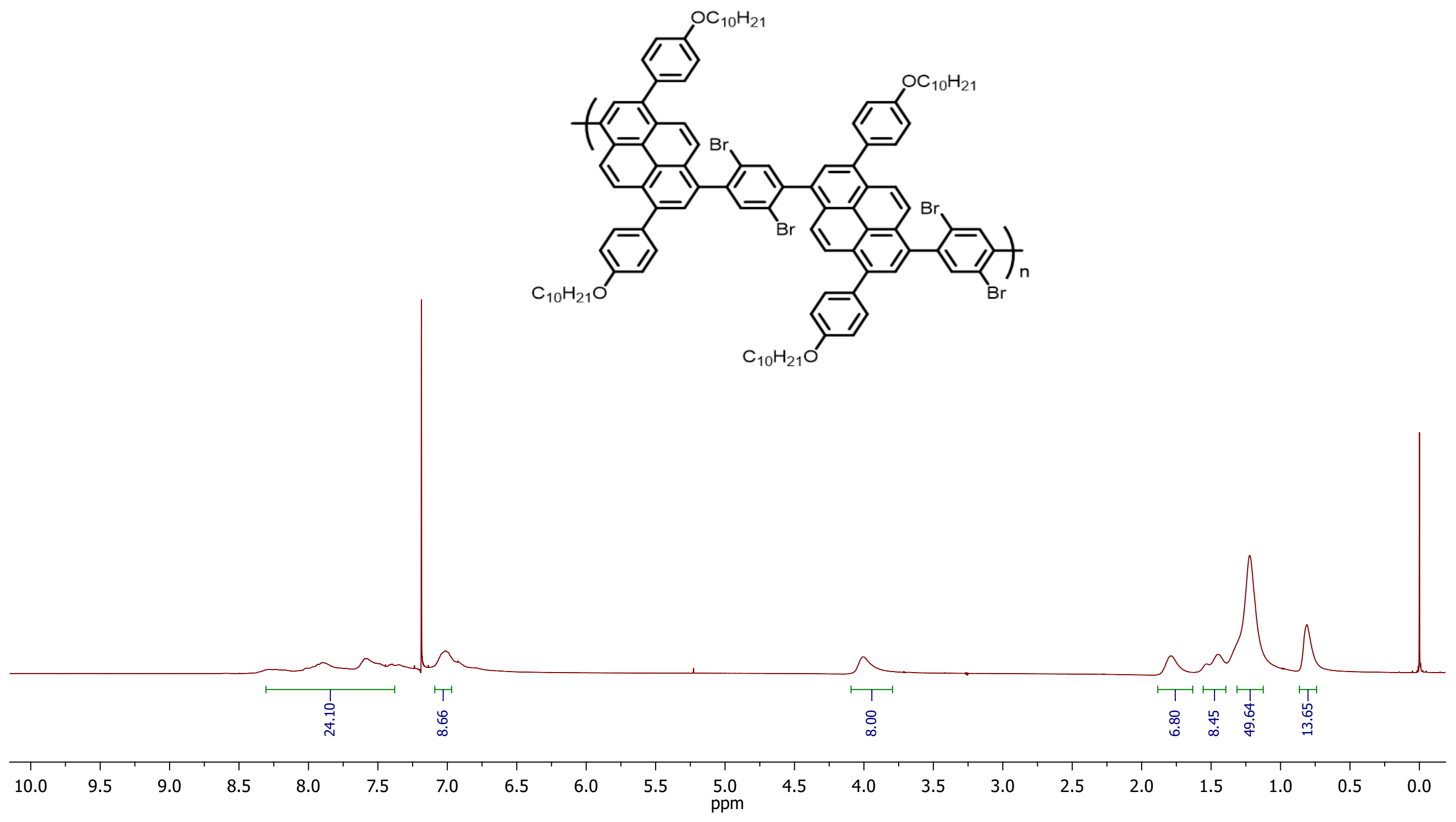


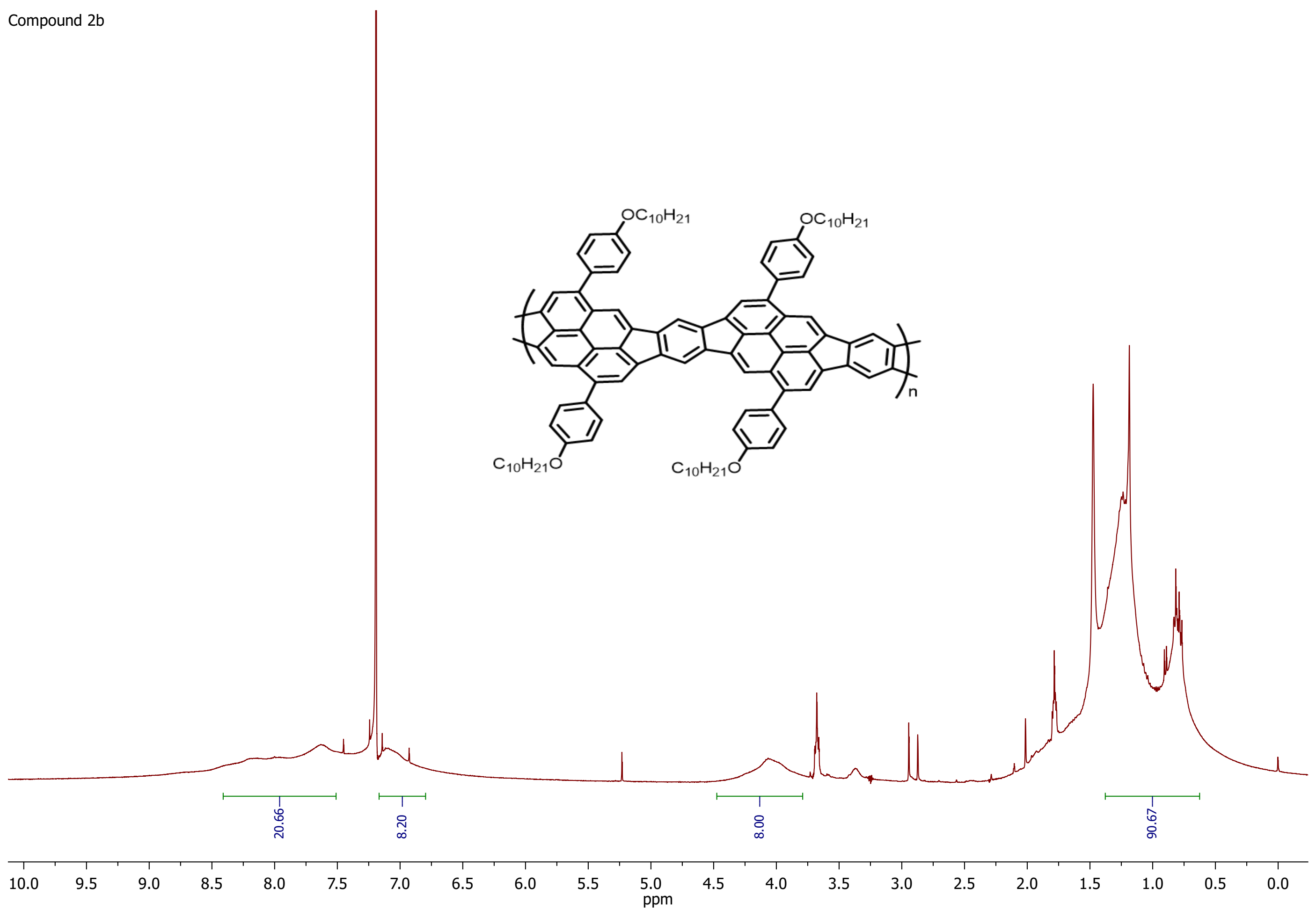

